\author{
UNIVERSIDADE DE SÃO PAULO \\ ESCOLA DE ENGENHARIA DE SÃO CARLOS \\ DEPARTAMENTO DE ENGENHARIA DE ESTRUTURAS
}

RAFAEL JOSE NIÑO TORO

PRESSÃO DE RUPTURA DE DUTOS CONTENDO DEFEITOS DE CORROSÃO

São Carlos

2014 



\section{PRESSÃO DE RUPTURA DE DUTOS CONTENDO DEFEITOS DE CORROSÃO}

Dissertação apresentada ao Departamento de Engenharia de Estruturas da EESC-USP como Parte integrante dos requisitos para Obtenção do titulo de Mestre em Engenharia de Estruturas.

Orientador: Prof. Dr. André Teófilo Beck

\section{Versão corrigida}

A versão original encontra-se na Escola de Engenharia de São Carlos

São Carlos

2014 
AUTORIZO A REPRODUÇÃO TOTAL OU PARCIAL DESTE TRABALHO, POR QUALQUER MEIO CONVENCIONAL OU ELETRÔNICO, PARA FINS DE ESTUDO E PESQUISA, DESDE QUE CITADA A FONTE.

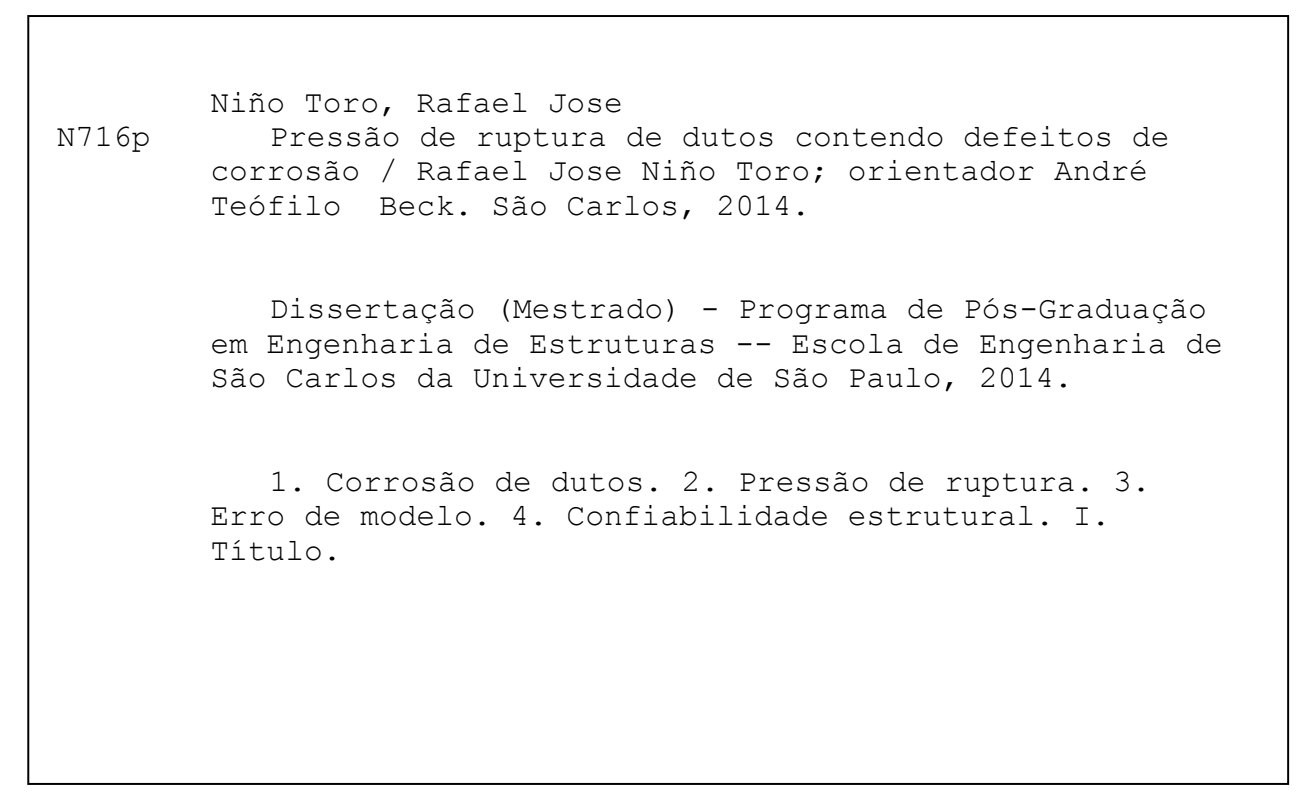




\section{FOLHA DE JULGAMENTO}

Candidato: Engenheiro RAFAEL JOSE NIÑO TORO.

Título da dissertação: "Pressão de ruptura de dutos contendo defeitos de corrosão".

Data da defesa: 17/10/2014

Comissão Julgadora:

Prof. Associado André Teófilo Beck (Orientador)

(Escola de Engenharia de São Carlos/EESC)

Prof. Dr. Edson Denner Leonel

(Escola de Engenharia de São Carlos/EESC)

Prof. Associado Gilberto Francisco Martha de Souza (Escola Politécnica/EP-USP)
Resultado:

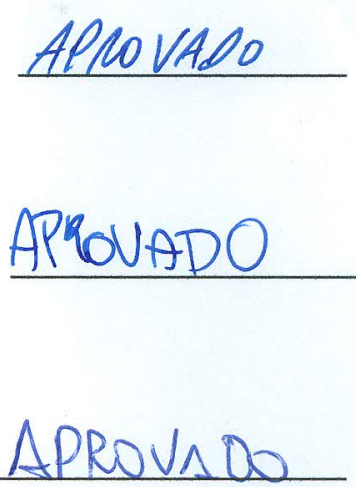

Coordenadora do Programa de Pós-Graduação em Engenharia Civil (Engenharia de Estruturas):

Profa. Associada Ana Lucia Homce de Cresce El Debs

Presidente da Comissão de Pós-Graduação:

Prof. Associado Paulo César Lima Segantine 

A mi mamá Marina, a mi padre

Hector, a mi hermana Karen Juliana y en especial a mi abuela Rosa Maria (In Memoriam) 



\section{AGRADECIMENTOS}

Agradeço primeiramente a Deus, pela sabedoria dada necessária para a conclusão desta etapa. A meus pais, Marina e Hector por seu amor incondicional, pelo seu grande apoio para conseguir meus objetivos, por toda sua paciência e dedicação e pela luta diária para eu conseguir me superar cada dia mais. A minha irmã, Karen Juliana, que me ajuda a ser cada dia uma melhor pessoa e tem me apoiado em todo momento. A minha avó, Rosa Maria (in memoriam) pelo seu grande amor, seu apoio para seguir em frente e ser um grande professional. A toda minha família por seu grande apoio e amor em todos os momentos difíceis da minha vida.

A Alanna por seu apoio e compreensão nesta etapa, apesar da distância e os momentos difíceis.

Ao professor André Beck, pelos ensinamentos, paciência, por seu apoio, incentivos, disponibilidade, que foram fundamentais para concluir este trabalho.

Ao professor Mario de Castro e Felipe Bazán, por sempre estarem dispostos para sanar minhas duvidas.

Aos professores Edson Denner Leonel e Rodrigo Paccola, por aceitarem a participação na banca examinadora, e pelas contribuições apresentadas no trabalho.

Aos meus amigos Ivan Gomez e Jesús Villalba, pela sua ajuda e incentivo a fazer o mestrado.

Aos amigos que eu fiz no Brasil especialmente no Departamento de Estruturas (SET): Andrezão, Arthur, Carlinhos, Cleilson, Daniel Bonfim, Elias, Emerson, Fernandão, Fernando Campo, Geovanne, Gian Franco, Greg, Hugão, João Marinho, Karen, Ketson, Lara, Marcell, Margot, Matheus, Nichollas, Pablo, Rafael Marques, Ricardo, Sergio, Thais.

Ao amigo e companheiro de apartamento, Amós (maluco) pelo aprendizado na convivência, companheirismo, pela ajuda em melhorar meu português, e por todo seu apoio em momentos de alegria e tristeza.

À CAPES pela bolsa de estudos concedida.

A todos meus amigos que não foram citados, mais que de uma forma contribuíram para o desenvolvimento do trabalho. 



\section{RESUMO}

TORO, R. J. N. Pressão de ruptura de dutos contendo defeitos de corrosão. 2014. Dissertação (Mestrado em Engenharia de Estruturas) - Escola de Engenharia de São Carlos, Universidade de São Paulo, São Carlos.

Uma grande variedade de modelos é utilizada para estimar a pressão de ruptura de dutos contendo defeitos de corrosão. O presente trabalho tem como objetivo estudar a precisão dos modelos mais comuns e avaliar a pressão de ruptura de dutos submetidos à corrosão. Os modelos avaliados são: ASME B31G, ASME B31G modificado, DNV RP F101 e PCORRC. O estudo é baseado em mais de 400 resultados de ensaios de ruptura em dutos corroídos, todos coletados da literatura. A base de dados contem defeitos de corrosão reais e artificiais. Uma análise estatística foi realizada para a variável erro de modelo. Uma análise de regressão não-linear foi realizada para investigar os efeitos da variável erro de modelo, das variáveis mais relevantes, como profundidade e comprimento do defeito, e tensão de ruptura do aço. Uma análise de confiabilidade foi realizada a partir das estatísticas obtidas da variável erro de modelo, sendo estimado o índice de confiabilidade e a probabilidade de falha do duto com defeitos de corrosão, através do método iterativo de primeira ordem, denominado FORM (“First Order Reliability Method”). Nesta análise avaliou-se a evolução da probabilidade de falha com o aumento da profundidade do defeito, bem como foram identificadas as variáveis aleatórias mais importantes na falha do duto. O estudo pode ajudar aos operadores a eleger qual modelo utilizar em análises de risco, proporcionando mais segurança às operações dutoviárias.

Palavras-chave: Corrosão de dutos. Pressão de ruptura. Erro de modelo. Confiabilidade estrutural. 



\begin{abstract}
TORO, R. J. N. On the burst pressure of pipelines containing corrosion defects. 2014. Master’s Thesis - São Carlos School of Engineering, University of São Paulo, São Paulo, 2014.
\end{abstract}

A variety of models exist to estimate burst pressures of pipelines containing corrosion defects. The objective of this work is to study the accuracy of some of the most popular empirical burst pressure models. The study addresses the models: ASME B31G, ASME B31G Modified, DNV RP-F101 and PCORRC. The investigation is based on over 400 burst test results, all collected from the literature, containing both real and artificial corrosion defects. A statistical analysis is performed for assessing the accuracy of semi-empirical models by using a model error variable. A non-linear regression analysis is performed to identify the influence, on model errors, of the most relevant variables, such as defect depth and length and steels rupture tension. A reliability analysis was carried out, using model error statistics developed herein, in order to evaluate reliability index and probability of failure of pipelines containing corrosion defects, through the iterative first order reliability method, or FORM - First Order Reliability Method.

The evolution of failure probabilities, with increasing defect depth, was investigated. The most relevant random variables were identified. The study can help operators choose a proper empirical model to use in their risk analysis, leading to greater safety in pipeline operations.

Keywords: Pipeline. Corrosion. Burst pressure. Model error. Structural reliability. 



\section{LISTA DE TABELAS}

Tabela 3.1. Parâmetros experimentais (Kiefner et al., 1973)................................................. 38

Tabela 3.2. Parâmetros experimentais (Vieth; Kiefner, 1994)................................................ 39

Tabela 3.3. Parâmetros experimentais (Stephens et al., 1995).................................................. 40

Tabela 3.4. Parâmetros experimentais (Benjamin et al., 2000)................................................41

Tabela 3.5. Parâmetros experimentais (Cronin; Pick, 2000)....................................................42

Tabela 3.6. Resumo de ensaios realizados (Mok et al., 1990). ...................................................43

Tabela 3.7. Parâmetros experimentais (Mok et al., 1990)...................................................... 43

Tabela 3.8. Parâmetros experimentais (Chouchaoui; Pick, 1992)............................................ 44

Tabela 3.9. Parâmetros experimentais para o primeiro conjunto (Chouchaoui; Pick, 1994)...45

Tabela 3.10. Parâmetros experimentais para o segundo conjunto (Chouchaoui; Pick, 1994). 46

Tabela 3.11. Parâmetros experimentais para o terceiro conjunto (Chouchaoui; Pick, 1996)...46

Tabela 3.12. Parâmetros experimentais (Roberts; Pick, 1998)................................................47

Tabela 3.13. Parâmetros experimentais (Nehoda; Horalek, 2000)..........................................48

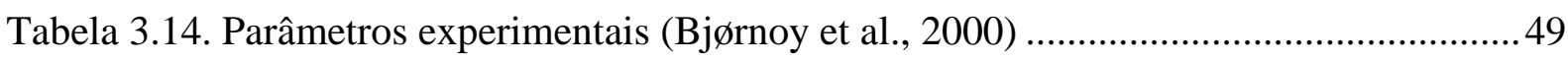

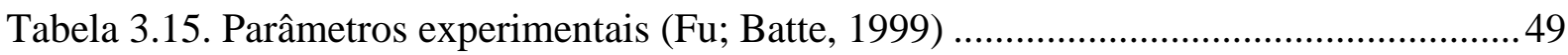

Tabela 6.1. Parâmetros estatísticos do teste Kolmogorov-Smirnov.........................................82

Tabela 6.2. Quantidade de dados utilizada para as análises.................................................... 83

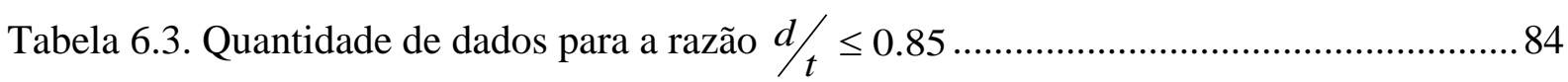

Tabela 6.4. Caracterização da Variável Erro de Modelo $(d / t \leq 0,85)$..................................85

Tabela 6.5. Estatística básica da variável erro de modelo (Zhou; Huang, 2012).....................87

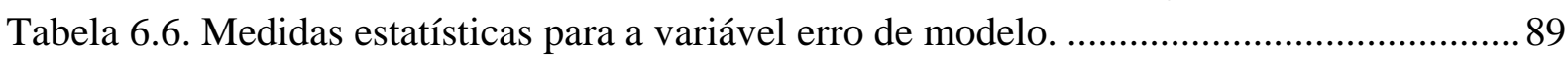

Tabela 6.7. Medidas estatísticas para a variável erro de modelo. ...........................................90

Tabela 6.8. Coeficientes da regressão não-linear obtidos para o modelo ASME B31G e DNV

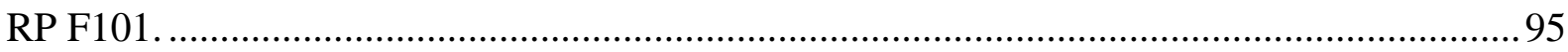

Tabela 6.9. Coeficientes do modelo de regressão múltipla....................................................96

Tabela 6.10. Coeficientes ajustados do modelo não-linear, DNV RP F101. .......................... 100

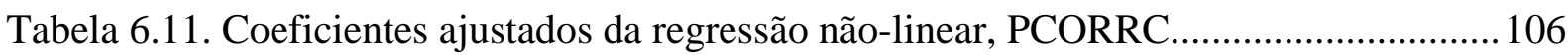

Tabela 6.12. Coeficientes ajustados da regressão não linear, modelo ASME B31G.............111

Tabela 6.13. Coeficientes ajustados de regressão, método B31G modificado........................116

Tabela 6.14. Caracterização das variáveis aleatórias do duto exemplo (Bazán e Beck, 2011).

Tabela 6.15. Resultados obtidos da análise de confiabilidade a partir do programa StRAnD. 



\section{SUMÁRIO}

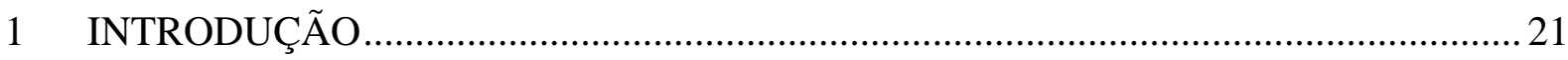

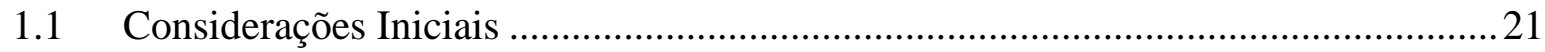

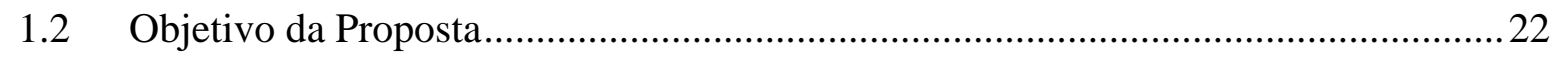

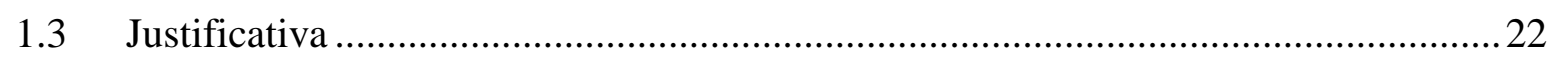

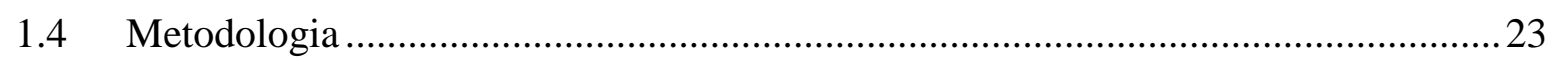

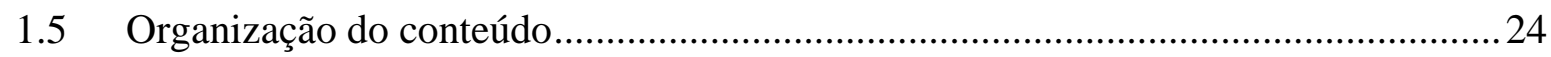

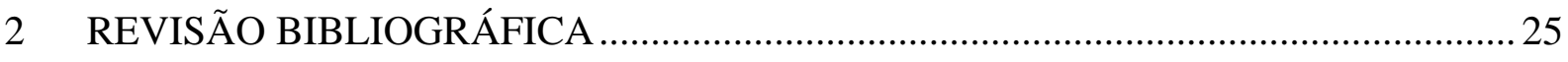

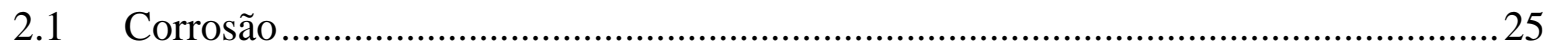

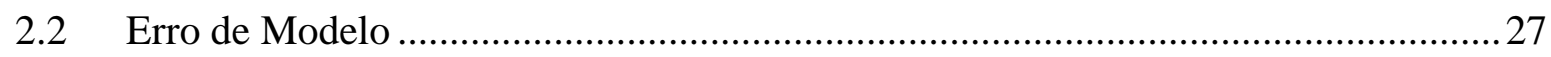

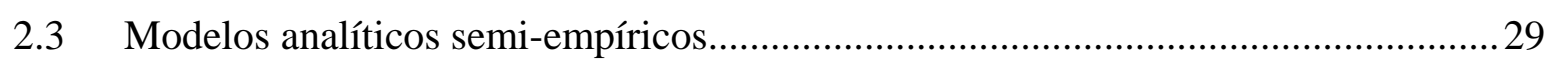

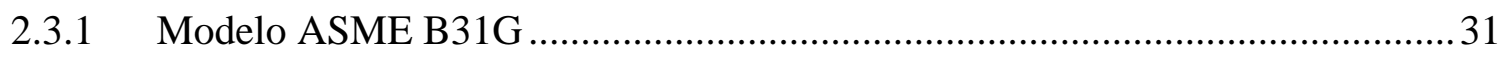

2.3.2 Modelo ASME B31G modificado ou 0.85dL ...................................................33

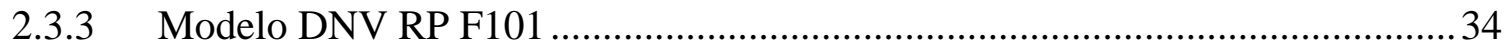

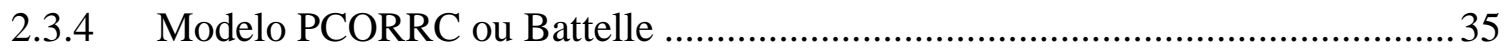

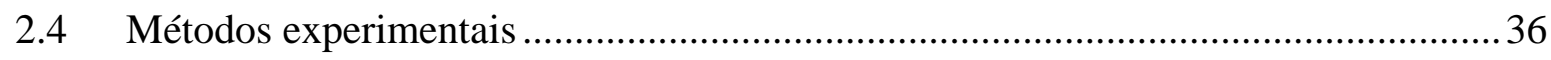

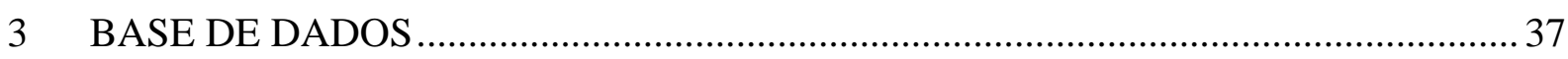

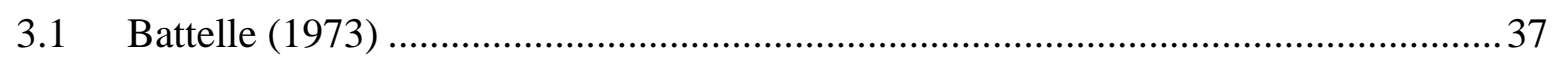

3.2 AGA/PRCI Database of Corroded Pipe Tests (1994)............................................38

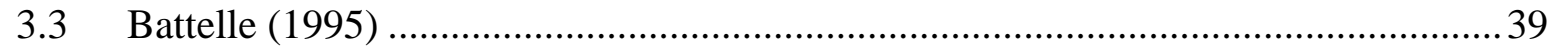

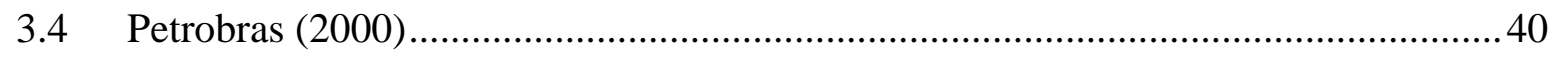

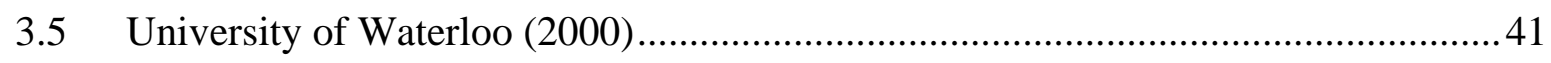

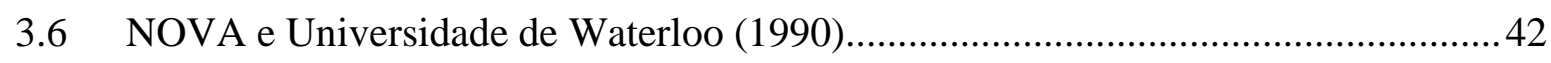

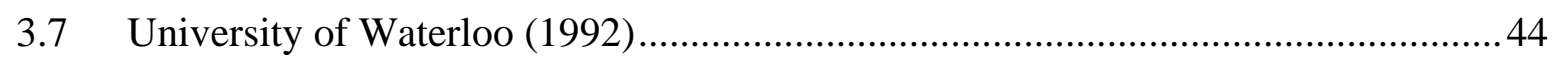

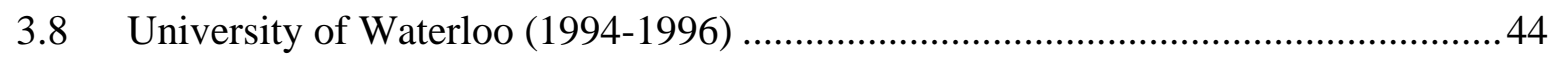

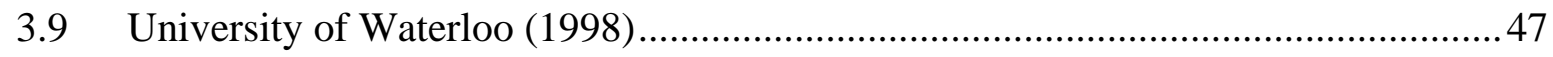

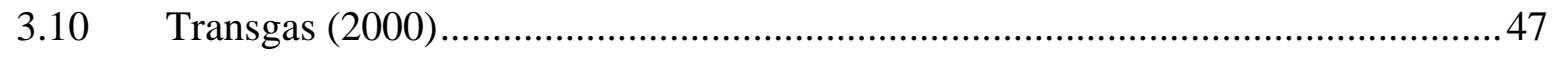

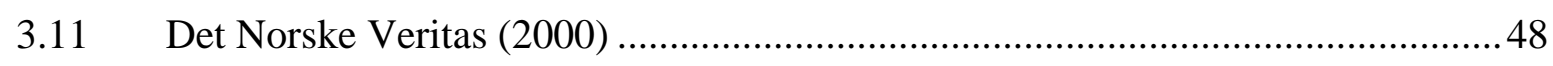

3.12 British Gas (1999) (Linepipe Corrosion Group Sponsored Project)..................... 48

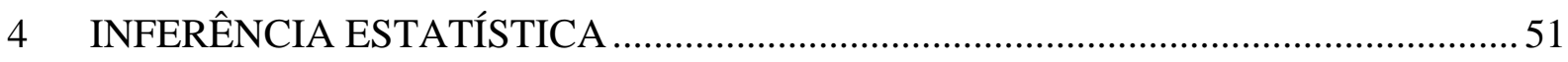

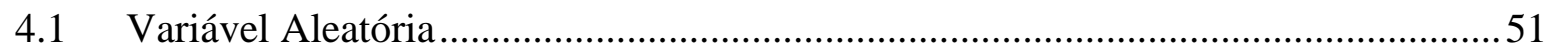

4.1.1 Função de distribuição acumulada de probabilidade............................................51 
4.1.2 Função de densidade de probabilidade ..............................................................52

4.1.3 Momentos de uma variável aleatória...............................................................52

4.1.4 Média e Variância de amostras............................................................................54

4.1.5 Distribuição de Probabilidade Normal ou Gaussiana..........................................54

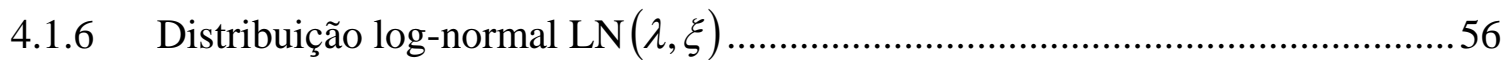

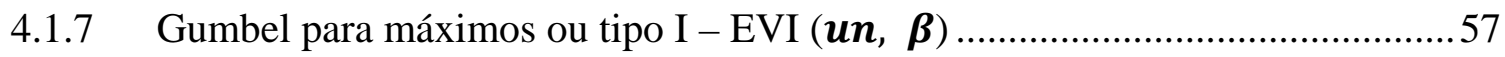

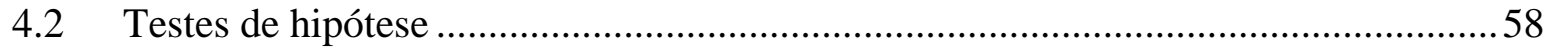

4.2.1 Teste de hipótese de Kolmogorov-Smirnov ........................................................ 58

4.2.2 Teste de hipótese de Anderson-Darling (A-D) ...................................................59

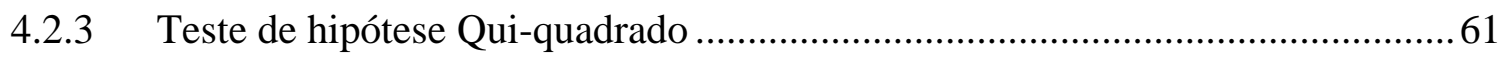

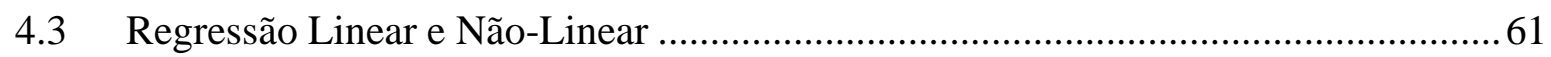

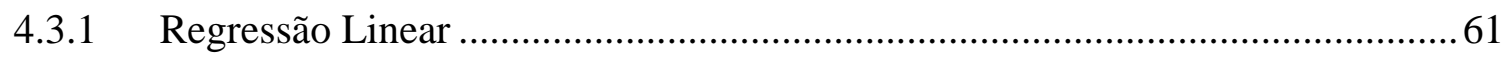

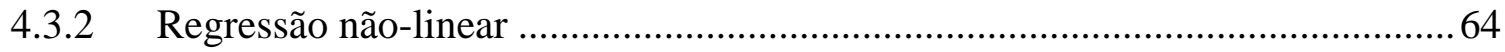

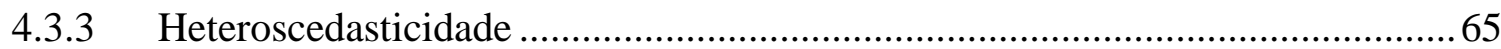

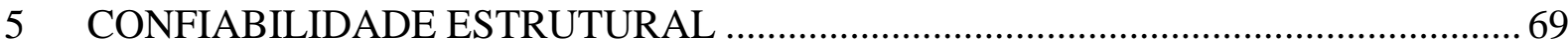

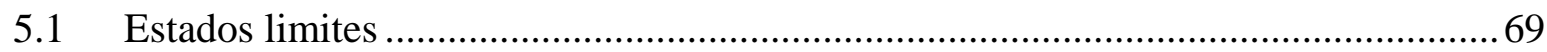

5.2 Problema fundamental de confiabilidade estrutural independente do tempo ............ 70

5.3 Aproximação de Primeira Ordem e Segundo Momento (FOSM) …...........................72

5.4 Algoritmo de Hasofer, Lind, Rackwitz e Fiessler....................................................74

5.4.1 Aproximação de Primeira Ordem (FORM)..................................................... 75

5.4.2 Transformação composta utilizando o modelo de Nataf ....................................76

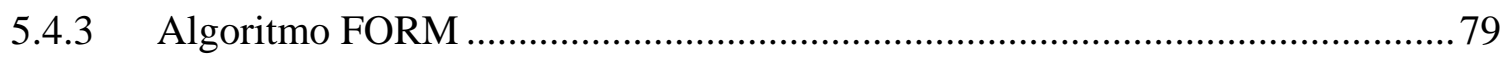

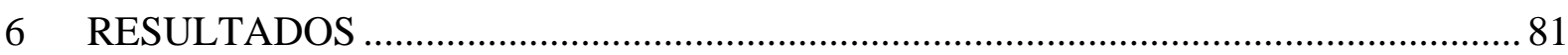

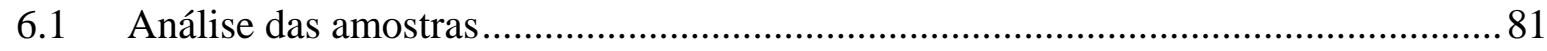

6.2 Caracterização da variável erro de modelo ........................................................... 84

6.3 Comparação com resultados da literatura ............................................................... 86

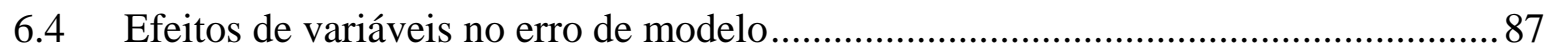

6.4.1 Erro de modelo versus resistência ao escoamento e resistência à tração última.88

6.4.2 Variável erro de modelo versus comprimento do defeito normalizado...............91

6.4.3 Análise de regressão não-linear múltipla..........................................................95

6.4.4 Modelos de regressão não lineares para erro de modelo versus profundidade

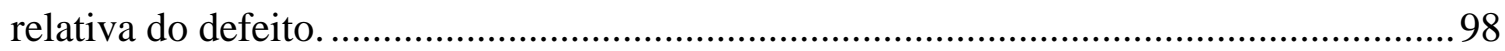

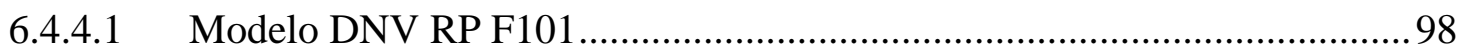




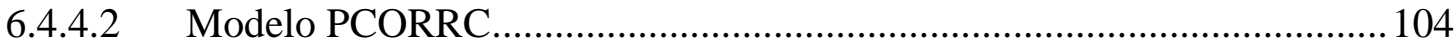

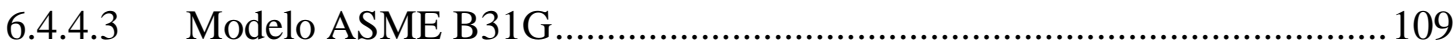

6.4.4.4 Modelo ASME B31G modificado ........................................................... 114

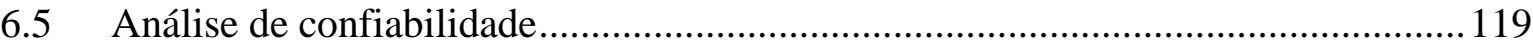

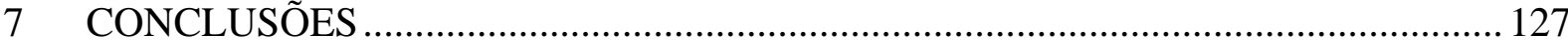

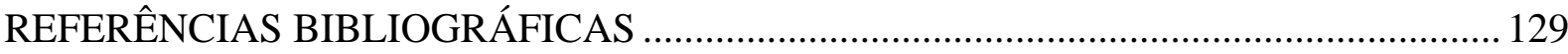

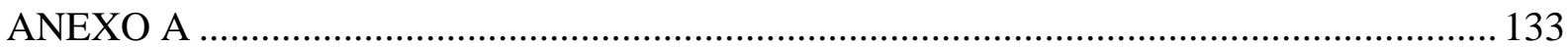





\section{INTRODUÇÃO}

\subsection{Considerações Iniciais}

Os dutos são utilizados na engenharia como meio de transporte de fluidos, sejam eles líquidos ou gasosos. A depender do fluido transportado eles podem ser denominados como: oleoduto, gasoduto ou poliduto.

Os dutos são construídos de acordo com normas internacionais de segurança para o transporte de substâncias. Eles estão sujeitos a efeitos, tais como corrosão ou deterioração mecânica do aço, que podem causar ruptura ou vazamentos. A corrosão é um dos principais mecanismos de falha em dutos enterrados, tornando necessária a reparação ou até a substituição de trechos de dutos.

A corrosão é um processo de degradação de um material, principalmente metal, por uma interação química ou eletroquímica com o meio ambiente (Gentil, 1996). Pode levar a um estado indesejável da estrutura, causando perdas econômicas, ambientais e humanas.

Para avaliar a pressão interna de falha de dutos foram desenvolvidos modelos analíticos semiempíricos como ASME B31G, ASME B31G modificado, DNV-RP-F101 e PCORRC. O modelo ASME B31G é o mais utilizado por ser o mais conservador e, portanto, a favor da segurança. Na maioria destes modelos não são consideradas as incertezas associadas às variáveis envolvidas. Elas são consideradas como determinísticas, o que impede que se garanta a segurança na estrutura. Utilizando os conceitos de confiabilidade estrutural é possível considerar as incertezas associadas às variáveis, e assim estimar a probabilidade de falha de dutos sujeitos à corrosão. Para estimar a probabilidade de falha será utilizado o método analítico de primeira ordem (FORM). 


\subsection{Objetivo da Proposta}

O objetivo principal desta pesquisa é avaliar a precisão dos modelos empíricos de previsão da pressão de ruptura de dutos contendo defeitos de corrosão.

Como objetivos específicos, tem-se:

- Analisar a precisão dos diferentes modelos semi-empíricos utilizados para estimar a pressão de ruptura (ASME B31G, ASME B31G modificado, DNV RP F101 e PCORRC).

- Confrontar os ensaios em dutos com defeitos reais e artificiais para verificar se pertencem a uma mesma população, para os diferentes modelos considerados.

- Avaliar a influência da profundidade relativa dos defeitos $(d / t)$ nos modelos de pressão de ruptura.

- Estudar a segurança de dutos, quando apresentam defeitos de corrosão.

- Analisar os efeitos da variável erro de modelo na análise de confiabilidade.

\subsection{Justificativa}

A variável erro de modelo é utilizada para representar as incertezas do modelo de pressão de ruptura. Esta variável aleatória é umas das mais relevantes no cálculo da probabilidade de falha. Por isso, é importante uma adequada representação probabilística da variável erro de modelo na análise de confiabilidade de dutos submetidos à corrosão.

Esta pesquisa se inspira em contribuir no âmbito cientifico no caminho de melhor representar o comportamento da variável erro de modelo, ajudando a obter uma melhor segurança ao se projetar dutos. 


\subsection{Metodologia}

Inicialmente é realizado um levantamento bibliográfico baseado em livros, artigos, dissertações, teses, textos técnicos e normativos correspondentes aos assuntos: corrosão de dutos, variáveis aleatórias, modelos de pressão de ruptura em dutos, erro de modelo, reabilitação de dutos, segurança nas estruturas, probabilidade de falha.

Seguidamente serão estudados diferentes métodos semi-empíricos de pressão de ruptura:

- ASME B31G

- ASME B31G modificado

- DNV-RP-F101

- PCORRC

Em seguida, a partir da análise de resultados experimentais e da avaliação da variável erro de modelo será estudada a precisão ou acurácia de cada um dos modelos semi-empíricos citados.

Finalmente a análise de confiabilidade será realizada por meio do código computacional StRAnD - Structural Reliability Analysis and Design (2007). Neste programa é necessário incluir as equações de estado limite específicas ao problema de dutos corroídos.

Por meio da análise de confiabilidade, pode-se determinar os índices de confiabilidade, probabilidade de falha e os coeficientes de sensibilidade, que são utilizados para determinar qual é a variável aleatória que faz a maior contribuição na probabilidade de falha no problema de dutos corroídos. 


\subsection{Organização do conteúdo}

No Capítulo 2 é apresentada a revisão bibliográfica, contendo uma breve introdução à respeito de corrosão em dutos. São abordadas as formulações teóricas e os modelos de predição da pressão de ruptura que serão utilizados no desenvolvimento do trabalho.

No Capitulo 3 são apresentadas as diferentes bases de dados coletadas da literatura, a partir das quais se fará as análises estatísticas para avaliação dos modelos semi-empíricos.

No Capítulo 4 são abordados os conceitos referentes à teoria estatística, que é de fundamental importância para o desenvolvimento deste trabalho, uma vez que a análise de confiabilidade requer de conhecimentos estatísticos.

No Capítulo 5 são abordados os conceitos que envolvem a confiabilidade estrutural.

No Capítulo 6 são apresentadas as análises de regressão para os modelos estudados. Será realizado um exemplo de cálculo para análise de confiabilidade estrutural.

No Capítulo 7 são apresentadas as conclusões e considerações finais. 


\section{REVISÃO BIBLIOGRÁFICA}

\subsection{Corrosão}

A corrosão é um fenômeno que se apresenta em todos os materiais existentes. Por definição tem-se que ela é a degradação de um material, principalmente metal, por uma interação química ou eletroquímica com o meio ambiente (Gentil, 1996).

A corrosão se deve a um processo químico chamado oxi-redução, o que significa que ocorre uma reação de oxidação e redução nos metais. Neste processo, o metal cede elétrons que são recebidos por qualquer substância (oxidante) que se encontre no meio corrosivo. A reação química que causa a corrosão produz deterioração no material espontaneamente, mudando suas propriedades e levando a perda da resistência e durabilidade. Segundo Gentil (1996), este fenômeno é de total importância na vida moderna, que não pode prescindir dos metais e suas ligas. Problemas de corrosão são frequentes em estruturas como: mineroduto, oleoduto, gasoduto, tanques de armazenamento de combustíveis como gasolina, álcool, óleo diesel, emissários submarinos.

A corrosão pode-se apresentar em variadas formas e pode ser classificada através de sua aparência no metal corroído. As principais características, para a classificação, são as seguintes (Figura 2.1):

- Uniforme: A corrosão encontra-se distribuída ao longo de toda a superfície da estrutura e é produzida pelo contato com o meio corrosivo, causando perda uniforme na espessura.

- Por placas: Localiza-se em alguns setores da superfície sendo não uniforme, formando placas ou cavações de profundidade constante. 
- Alveolar: Manifesta-se na superfície metálica produzindo cavidades com fundo arredondado e profundidade menor que o seu diâmetro.

- Puntiforme ou por pites: caracteriza-se por ser uma forma localizada na superfície metálica, comumente seu fundo tem forma angulosa e sua profundidade é maior que do que seu diâmetro.

- Intergranular ou intercristalina: A corrosão se produz entre os grãos da rede cristalina do material metálico, o qual perde suas propriedades mecânicas e pode fraturar quando solicitado por esforços mecânicos, tendo-se a corrosão sob tensão fraturante.

- Intrangular ou transgranular ou transcristalina: A corrosão se produz nos grãos da rede cristalina que perdendo suas propriedades mecânicas, pode fraturar a baixa solicitação mecânica, tendo-se também corrosão sob tensão fraturante.

- Por esfoliação: A corrosão se processa de forma paralela à superfície metálica. Este tipo de corrosão ocorre em chapas, cujos grãos foram achatados ou alongados.
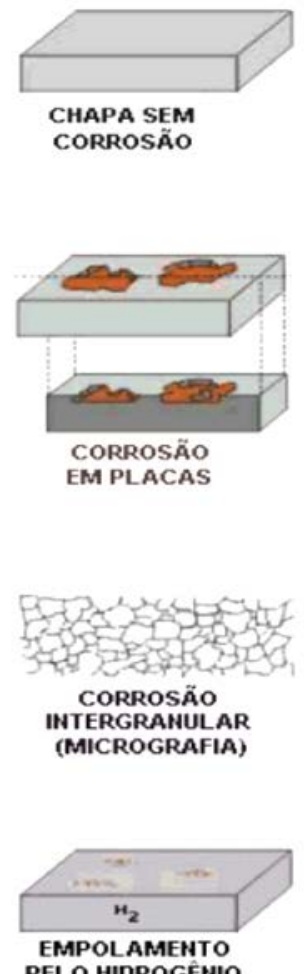

PELO HIDROGÊIO
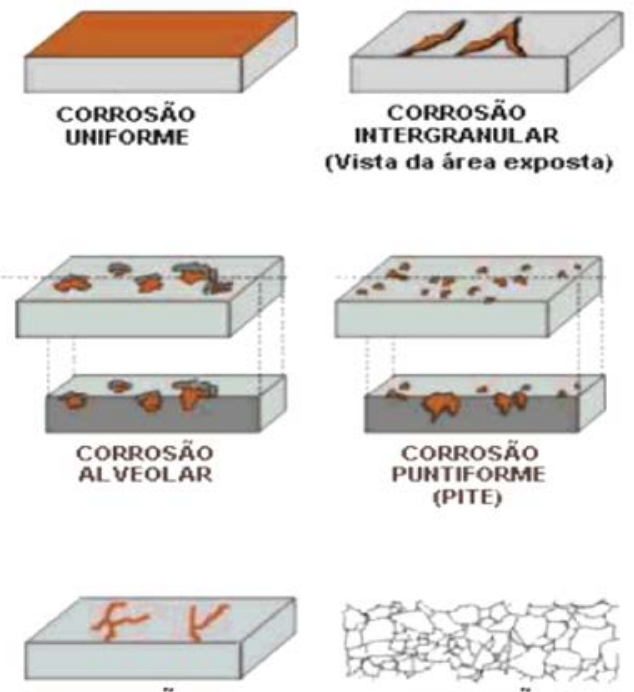

CORROSÄO FILIFORME

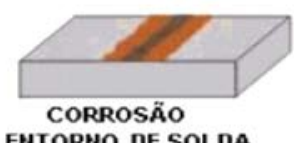

EHTORHO DE SOLDA
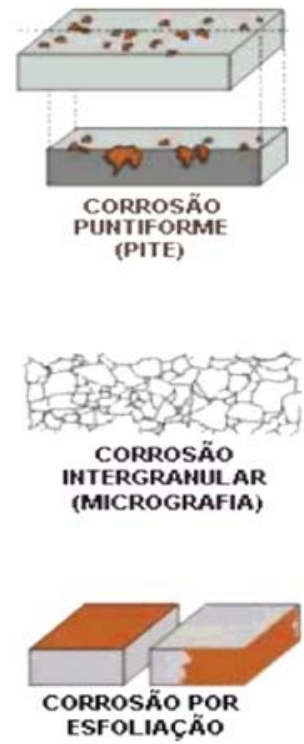

Figura 2.1. Formas de corrosão. [Abraco, Disponível em:

$<$ http://paginapessoal.utfpr.edu.br/israel/teoria/Teoria_Corrosao_Abraco.pdf/at_downl oad/file $>$ Acesso em: 14/02/2013]. 


\subsection{Erro de Modelo}

A variável erro de modelo é obtida a partir de uma comparação entre resultados experimentais, obtidos em laboratório por meio de ensaios (full-scale) e valores teóricos, determinados a partir dos modelos semi-empíricos de pressão de ruptura. A Equação (2.1) apresenta a relação matemática para se determinar a variável aleatória erro de modelo:

$$
X_{M}=\frac{P_{\text {Experimental }}}{P_{\text {Modelo }}},
$$

considerando-se $X_{M}$ como uma variável aleatória que representa o fator do erro de modelo, $P_{\text {Experimental }}$ o valor obtido dos ensaios de laboratório (full-scale) e $P_{\text {Modelo }}$ o valor obtido a partir dos modelos de predição de ruptura.

Determinada a variável aleatória erro de modelo, realiza-se a sua caracterização. Isto é realizado através da construção de um histograma, que ajuda a encontrar a distribuição de probabilidades que melhor representa o comportamento dos dados. Ressalta-se que é necessário realizar testes de aderência e ajustes para obter informações estatísticas do modelo, tais como: o valor médio $(\mu)$ e o desvio padrão $(\sigma)$. O valor médio representa o viés do modelo, podendo ser conservador ou inseguro, enquanto o desvio padrão $(\sigma)$ representa a dispersão do modelo. Para uma adequada representação do fenômeno físico estudado, os dados devem resultar em uma média próxima a um valor unitário.

Com o valor médio da variável erro de modelo é possível realizar a correção do modelo matemático $\left(P_{\text {Modelo }}^{\text {Corr }}\right.$ ). Este valor médio pode ser obtido por métodos estatísticos ou por meio de uma análise de regressão. O modelo de regressão é utilizado quando duas ou mais variáveis estão relacionadas. Quando existe alguma relação entre as variáveis, é necessário pensar em um modelo de regressão empírico, dado por:

$$
X_{M}(x)=\beta_{0}+\beta_{1} \cdot x+\varepsilon,
$$


onde $\beta_{0}$ é a interseção, $\beta_{1}$ é a inclinação, sendo parâmetros a determinar e $\varepsilon$ é o erro aleatório do modelo, com média nula e variância constante $\sigma^{2}$ a ser determinada. Aplicando o operador valor esperado, obtém-se:

$$
\begin{aligned}
& E\left[X_{M}(x)\right]=E\left[\beta_{0}+\beta_{1} \cdot x+\varepsilon\right], \\
& E\left[X_{M}(x)\right]=E\left[\beta_{0}+\beta_{1} \cdot x\right]+E[\varepsilon], \\
& E\left[X_{M}(x)\right]=E\left[\beta_{0}+\beta_{1} \cdot x\right]+0=\beta_{0}+\beta_{1} \cdot x .
\end{aligned}
$$

Aplicando o operador variância, tem-se:

$$
\begin{aligned}
& \operatorname{Var}\left[X_{M}(x)\right]=\operatorname{Var}\left[\beta_{0}+\beta_{1} \cdot x+\varepsilon\right], \\
& \operatorname{Var}\left[X_{M}(x)\right]=\operatorname{Var}\left[\beta_{0}+\beta_{1} \cdot x\right]+\operatorname{Var}[\varepsilon], \\
& \operatorname{Var}\left[X_{M}(x)\right]=0+\operatorname{Var}[\varepsilon]=0+\sigma^{2}=\sigma^{2} .
\end{aligned}
$$

Portanto, para se determinar a correção do modelo $\left(P_{\text {Modelo }}^{\text {Corr }}\right)$ é utilizada a Equação (2.5) para assim obter:

$$
P_{\text {Modelo }}^{\text {Corr }}=\left[\beta_{0}+\beta_{1} \cdot x\right] \cdot P_{\text {Modelo }} .
$$

Também pode ser determinada uma variável erro de modelo para o modelo corrigido:

$$
X_{M}^{\text {Corr }}=\frac{P_{\text {Experimental }}}{P_{\text {Modelo }}^{\text {Corr }}}
$$

É importante destacar que serão utilizados os modelos de regressão, para assim obter os valores médios da variável erro de modelo (Equação (2.5)). Isto é fundamental para as análises de confiabilidade que serão realizadas nos capítulos posteriores. 


\subsection{Modelos analíticos semi-empíricos}

Os modelos semi-empíricos são baseados nos conceitos da mecânica da fratura e em ensaios experimentais que determinam a pressão de ruptura dos dutos. Dentre os diversos modelos encontrados na literatura, tem-se o ASME B31G (American Society of Mechanical Engineers), ASME B31G modificado ou 085dL, o DNV RP F101 (Det Norske Veritas Recommended Practice RP-101 Corroded Pipelines) e o PCORRC (Battelle). Pode-se considerar o avanço do defeito a partir da estimativa de uma taxa de corrosão. A Figura 2.2 ilustra um defeito de corrosão arbitrário em um duto.
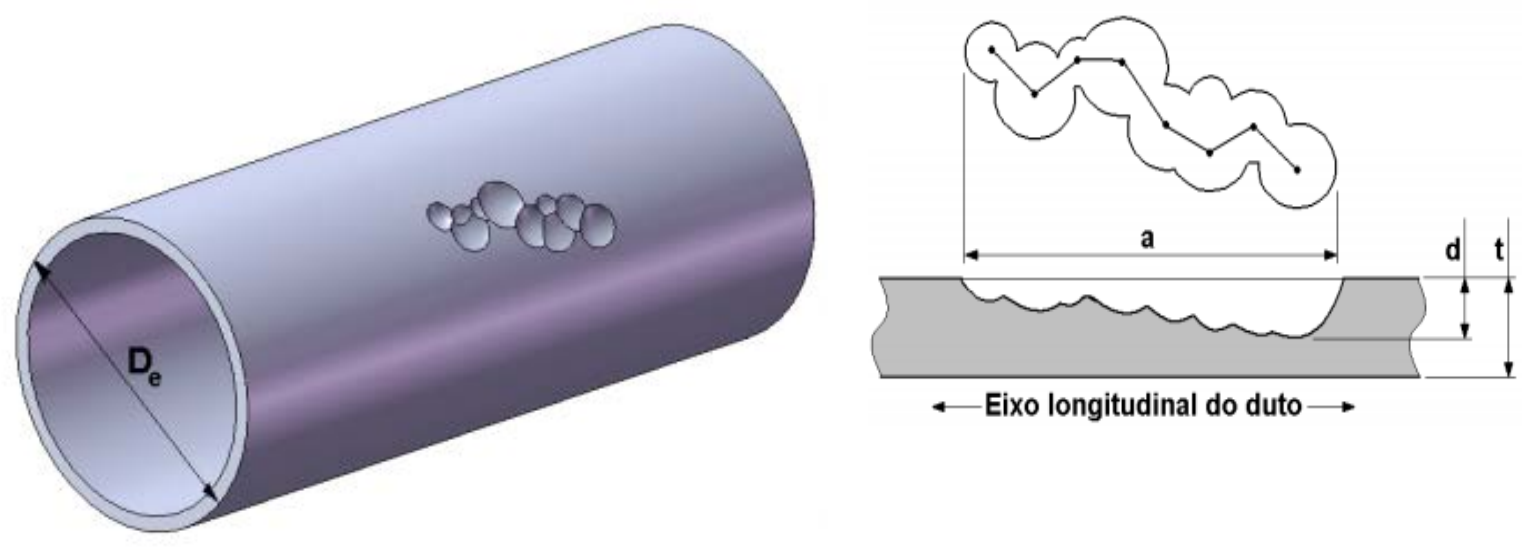

Figura 2.2. Representação de defeitos de corrosão em dutos [Cordeiro, 2009].

A formulação básica dos modelos é baseada nos critérios da equação NG-18 Surface Flaw Equation. Segundo Escoe (2006) este nome vem de um trabalho patrocinado pela NG-18 Line Pipe Research Committee of the American Gas Association (AGA). A equação NG-18 Surface Flaw Equation descreve uma relação entre a tensão de fluxo no cilindro pressurizado e o tamanho do defeito:

$\sigma_{\text {rup }}=\sigma_{\text {flow }} \cdot f_{R}$,

onde $\sigma_{\text {rup }}$ é a tensão de ruptura, $\sigma_{\text {flow }}$ é a tensão de fluxo, $f_{R}$ é o fator de redução. O fator de redução é obtido pela seguinte equação: 


$$
f_{R}=\left[\frac{1-\alpha \cdot \frac{A}{A_{0}}}{1-\alpha \cdot\left(\frac{A}{A_{0}}\right) M^{-1}}\right],
$$

onde $A_{0}=L \cdot t$ é área original da região corroída, $A=L \cdot d$ é a área corroída, $L$ é o comprimento do defeito, $d$ é a profundidade máxima do defeito, $M$ é o fator de dilatação (fator Faolias ou fator “bulging”). O fator de dilatação foi criado para levar em consideração a deformação em forma de bolha na tensão circunferencial aplicada na região corroída. A tensão circunferencial ou “hoop stress” $\left(\sigma_{\text {cic }}\right)$ é obtida a partir de uma relação da pressão interna do duto pela área da seção transversal da parede:

$\sigma_{\text {cic }}=\frac{P D}{2 t}$

No estado limite, considera-se que a pressão interna $(p)$ é igual à pressão de falha $\left(P_{\text {fail }}\right)$, e consequentemente $\sigma_{\text {cic }}=\sigma_{\text {rup }}$. Portanto, a pressão de falha é dada por:

$$
P_{\text {fail }}=\frac{2 \sigma_{\text {rup }} t}{D}
$$

Substituindo a Equação (2.11) na (2.14) chega-se a:

$$
P_{\text {fail }}=\frac{2 \cdot t \cdot \sigma_{\text {flow }} \cdot f_{R}}{D}
$$

Substituindo a Equação (2.12) na (2.15) determina-se a pressão de falha do duto:

$$
P_{\text {fail }}=\frac{2 \cdot t \cdot \sigma_{\text {flow }}}{D}\left[\frac{1-\alpha \cdot\left(\frac{d}{t}\right)}{1-\alpha \cdot\left(\frac{d}{t}\right) M^{-1}}\right] \text {, }
$$


onde $P_{\text {fail }}$ é a pressão de ruptura do duto, $t$ é a espessura da parede, $D$ é o diâmetro externo do duto, $\alpha$ é um fator empírico, $d$ é a profundidade da corrosão, $M$ é o fator de dilatação (fator Folias ou fator “bulging”). O fator de dilatação é função do comprimento do defeito, dividido pela espessura e pelo diâmetro do tubo, sendo adimensional.

A Equação (2.15) é dependente das propriedades do material do duto $\left(\sigma_{\text {flow }}\right)$, da geometria e características do defeito $\left(f_{R}\right)$. Ressalta-se que $\sigma_{\text {flow }}$ é proporcional à tensão de escoamento $\left(\sigma_{\text {esc }}\right)$ ou à tensão à tração última $\left(\sigma_{u}\right)$, e pode variar dependendo do material e do modelo utilizado. Os modelos semi-empíricos citados no presente trabalho serão apresentados nos sub-itens seguintes.

\subsubsection{Modelo ASME B31G}

O modelo ASME B31G foi desenvolvido a partir do critério da NG-18 Surface Flaw Equation. Este modelo considera a forma irregular do defeito de corrosão, como um perfil em forma parabólica. A partir do perfil parabólico, tem-se a área de perda de metal definida para defeitos curtos como $\alpha=2 / 3 d L$ (Figura 2.3a). Quando o comprimento do defeito aumenta, a representação parabólica da área de perda de metal se torna menos precisa. Porém, para defeitos longos a ASME B31G considera a área de perda de metal como um perfil retangular com $\alpha=1$ (Figura 2.3b).

No defeito, a tensão de fluxo no instante da ruptura é proporcional à tensão de escoamento, e é calculada como: $\sigma_{\text {flow }}=1,10 \cdot \sigma_{\text {esc }}$, onde $\sigma_{\text {esc }}$ é a tensão de escoamento do material, em MPa. 


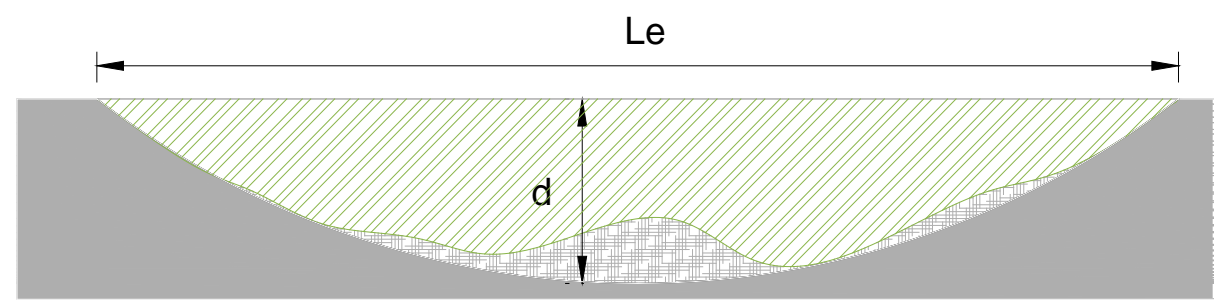

(a)

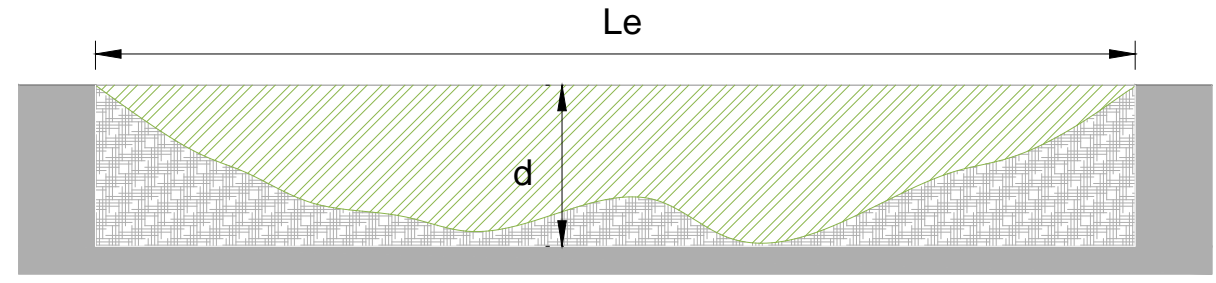

(b)

Corrosão $\square$ Area definida pela parabola $\quad \square$ Duto

Figura 2.3. Forma geométrica utilizada para representar a área de material perdido através da constante. (a) $\alpha=2 / 3$. (b) $\alpha=1$.

As Equações (2.17) e (2.18) são fornecidas pela ASME B31G para o cálculo da pressão de falha em dutos.

- Um defeito é considerado curto quando satisfaz a condição: $L \leq \sqrt{20 \cdot D \cdot t}$. Neste caso, a pressão de falha é:

$P_{\text {fail }}=1,10 \cdot \sigma_{e s c} \frac{2 \cdot t}{D}\left[\frac{1-\frac{2}{3}\left(\frac{d}{t}\right)}{1-\frac{2}{3}\left(\frac{d}{t}\right) M^{-1}}\right]$.

- Um defeito é considerado longo quando satisfaz a condição: $L \geq \sqrt{20 \cdot D \cdot t}$. Ao satisfazer a condição do comprimento, o fator de dilatação tende a infinito $(M \rightarrow \infty)$, significando que os defeitos são considerados infinitamente longos. Assim, obtém-se a pressão de falha:

$$
P_{\text {fail }}=1,10 \cdot \sigma_{\text {esc }} \cdot \frac{2 \cdot t}{D} \cdot\left(1-\frac{d}{t}\right) \text {, }
$$


onde o fator de dilatação, $M$, é obtido como:

$$
M=\sqrt{1+0,8\left(\frac{L^{2}}{D \cdot t}\right)} .
$$

Ressaltasse que para defeitos de corrosão com perda de espessura da parede entre $10 \%$ e $80 \%$ o duto deve ser reparado ou substituído segundo a ASME B31G. Esta limitação não contaminam os resultados obtidos no Capitulo 6, devido a que o volumem de dados é muito baixo.

\subsubsection{Modelo ASME B31G modificado ou 0.85dL}

O modelo ASME B31G modificado foi desenvolvido porque o modelo B31G apresentava resultados excessivamente conservadores. Kiefner e Vieth (1989) introduziram duas principais mudanças, na tensão de fluxo e no fator de dilatação. O fator de dilatação foi modificado para obter valores mais exatos e uma pressão de falha menos conservadora. Também apresentaram uma nova geometria para o defeito de corrosão. Esta geometria tem um formato entre o parabólico e retangular. O fator empírico utilizado para a geometria é $\alpha=0,85 \cdot d L$ (Figura 2.4).

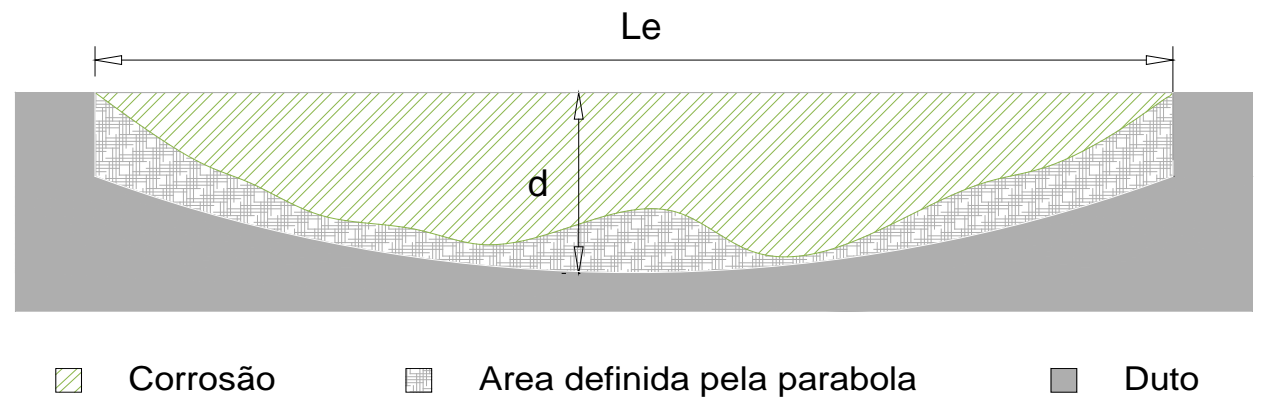

Figura 2.4. Forma geométrica utilizada para representar a área do material perdido através da constante $\alpha=0,85$.

Para Kiefner e Vieth (1989) a tensão de fluxo deve ser obtida da seguinte forma: $\sigma_{\text {flow }}=\sigma_{\text {esc }}+68,95 \mathrm{MPa}$. Antes de se determinar a pressão de falha, deve-se analisar se o defeito é considerado curto ou longo, como segue: 
- Um defeito é considerado curto, quando satisfaz a condição: $L \leq \sqrt{50 \cdot D \cdot t}$. Neste caso, o fator de dilatação é obtido como:

$$
M=\sqrt{1+0,6275 \cdot \frac{L^{2}}{D \cdot t}+0,003375 \cdot \frac{L^{4}}{(D \cdot t)^{2}}} .
$$

- Um defeito é considerado longo, quando satisfaz a condição: $L \geq \sqrt{50 \cdot D \cdot t}$. Neste caso, é obtido o fator de dilatação como:

$$
M=3,3+0,032 \cdot \frac{L^{2}}{D \cdot t} .
$$

Portanto, neste modelo a pressão de falha é determinada por:

$$
P_{\text {fail }}=\left(\sigma_{e s c}+68,95\right) \cdot \frac{2 \cdot t}{D} \cdot\left[\frac{1-0,85 \cdot\left(\frac{d}{t}\right)}{1-0,85 \cdot\left(\frac{d}{t}\right) M^{-1}}\right] \text {. }
$$

Ressaltasse que para defeitos de corrosão com perda de espessura da parede entre $20 \%$ e $80 \%$ o duto deve ser reparado ou substituído segundo a ASME B31G. Para esta limitação o volumem de dados é muito baixo, não contaminando os resultados obtidos no Capitulo 6.

\subsubsection{Modelo DNV RP F101}

As equações empíricas desenvolvidas pela Det Norske Veritas foram validadas através de análises de elementos finitos e por meio de 138 ensaios a escala real (full-scale), incluindo defeitos reais e artificiais (usinados). O modelo utiliza um fator empírico de $\alpha=1$ (Figura 2.3b) e uma tensão de fluxo $\sigma_{\text {flow }}=\sigma_{u}$, sendo $\sigma_{u}{ }^{*}$ tensão à tração última (MPa).

A seguir é apresentada a equação para obter a pressão de falha:

* Os modelos baseados em tensão à tração última são aplicáveis para todos os aços classe X 
$P_{\text {fail }}=\frac{2 \cdot \sigma_{u} \cdot t}{D-t} \cdot\left[\frac{1-\left(\frac{d}{t}\right)}{1-\left(\frac{d}{t}\right) M^{-1}}\right]$,

onde o fator de dilatação, $M$, é obtido como:

$$
M=\sqrt{1+0,31 \cdot \frac{L^{2}}{D \cdot t}} .
$$

\subsubsection{Modelo PCORRC ou Battelle}

O laboratório Battelle empreendeu uma investigação para o PRCI, a fim de realizar estudos sobre defeitos geométricos, pressão combinada, e carregamento axial em dutos com defeitos causados pelo processo da corrosão. Os pesquisadores conduziram ensaios à escala real (fullscale), investigação por elementos finitos e análise teórica, descobrindo que materiais mais dúcteis falham por colapso plástico. Battelle desenvolveu um software de elementos finitos conhecido como PCORR, que avalia a corrosão em um duto sujeito a pressão interna ou carregamento axial. Os pesquisadores também desenvolveram uma alternativa para avaliar um defeito de corrosão, por meio de uma análise de regressão de resultados obtidos através de um estudo paramétrico utilizando o software PCORR. O modelo semi-empírico desenvolvido ficou conhecido como PCORRC (Pipe CORRosion failure Criterion). Neste modelo a tensão de fluxo é igual à tensão à tração última $\sigma_{\text {flow }}=\sigma_{u}$. A pressão de falha a partir do modelo PCORRC é obtida como:

$$
P_{\text {fail }}=\frac{2 \cdot t \cdot \sigma_{u}}{D}\left(1-\frac{d}{t} \cdot M\right)
$$

onde o fator de dilatação, $M$, é obtido da maneira a seguir:

$$
M=1-\exp \left(\frac{-0,157 \cdot L}{\sqrt{\frac{D \cdot(t-d)}{2}}}\right) .
$$


É importante destacar que este método é o único que não é baseado na equação NG-18 e os critérios de falha são diferentes.

\subsection{Métodos experimentais}

Os ensaios experimentais são realizados em dutos que apresentam defeitos de corrosão (burst test) e seu objetivo principal é determinar a pressão de falha ou pressão de ruptura do duto. Nestes ensaios os tubos são fechados com tampas soldadas em suas extremidades e pressurizados internamente com água (Figura 2.5). As pressões e deformações são obtidas ao longo do ensaio, através de manômetros e extensômetros (strain gages).

A ruptura ocorre na região do defeito de corrosão, devido à concentração de tensões atuantes. Em materiais de média e elevada resistência, estas tensões conduzem a ruptura dos dutos por colapso plástico.

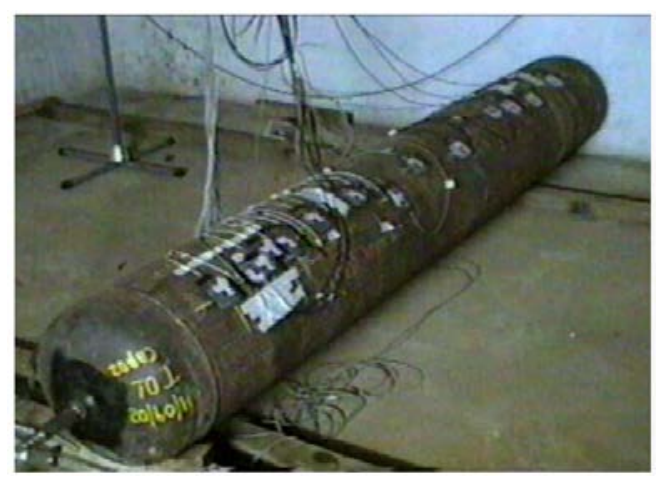

(a)

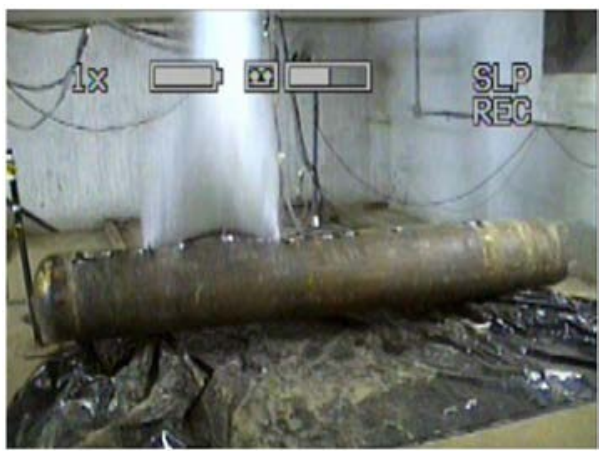

(b)

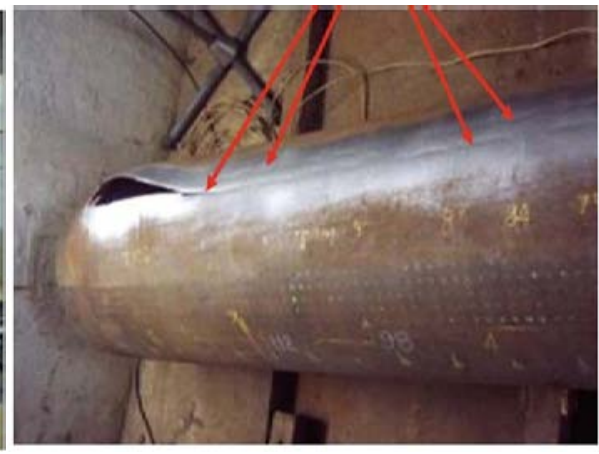

(c)

Figura 2.5. Equipamentos de ensaios de pressão de ruptura em um duto. (a) Instrumentação para as medições. (b) Ruptura de um duto. (c) Detalhe da ruptura na região do defeito.

[Benjamin et al., 2004]. 


\section{BASE DE DADOS}

Para estudar a precisão dos modelos de previsão de ruptura, comparam-se suas previsões com a pressão de ruptura obtida em ensaios em escala real (full-scale) de dutos contendo defeitos de corrosão. Estes defeitos podem ser: defeitos reais, produzidos pelo processo de corrosão em dutos retirados de serviço ou podem ser defeitos artificiais, produzidos por usinagem.

Os dados coletados no presente trabalho possuem defeitos com diferentes geometrias e materiais com distintas propriedades mecânicas. A base de dados é constituída por um conjunto de 427 ensaios, contendo defeitos de corrosão reais e artificiais. A principal dificuldade de se montar a base de dados foi a de extrair da literatura as condições exatas do ensaio e do tubo ensaiado, as quais nem sempre estão disponíveis ou não foram citados nos trabalhos publicados. A seguir descreve-se a base de dados considerada na pesquisa.

\subsection{Battelle (1973)}

Kiefner et al. (1973) realizaram 47 ensaios de ruptura (burst test) em dutos que continham diferentes geometrias de defeitos de corrosão reais. Estes ensaios foram realizados em corpos de prova de dutos removidos de serviço depois de alguns anos de operação. As características dos corpos de prova nos ensaios realizados são: 11 ensaios em dutos de grau X52 de 762 mm de diâmetro externo, 9,52 mm de espessura da parede; 20 ensaios em dutos grau B de 609,6 mm de diâmetro externo, 9,52 mm de espessura da parede; 9 ensaios em dutos grau B de 609,6 mm de diâmetro, 7,92 mm de espessura da parede; 2 ensaios em dutos de grau B de 508 mm de diâmetro, 7,87 mm de espessura da parede e 5 ensaios em dutos de grau A-25 de 406,4 mm de diâmetro externo, 7,92 mm de espessura da parede. Estes ensaios de ruptura compõem a base do desenvolvimento dos modelos de falha para dutos corroídos da ASME B31G. Os parâmetros experimentais são listados na Tabela 3.1. 
Tabela 3.1. Parâmetros experimentais (Kiefner et al., 1973).

\begin{tabular}{|c|c|c|c|}
\hline Diâmetro externo do duto (D), mm & 406,4 & $\mathrm{a}$ & 762,0 \\
\hline Espessura da parede (t), mm & 7,87 & a & 9,65 \\
\hline Relação 2R/t & 51,3 & a & 81,1 \\
\hline Grau (API 5L) & $A-25$ & a & $\mathrm{X} 52$ \\
\hline Resistência ao escoamento (Y), $\mathrm{Nmm}^{-2}$ & 195,8 & a & 508,9 \\
\hline Resistência à tração $(\mathrm{T}), \mathrm{Nmm}^{-2} *$ & 277,2 & $\mathrm{a}$ & 595,7 \\
\hline $\mathrm{Y} / \mathrm{T}$ & 0,58 & $\mathrm{a}$ & 0,79 \\
\hline 2/3 Energia de Impacto Charpy, J* & 31,0 & $\mathrm{a}$ & 45,0 \\
\hline Transmissão de temperatura, C & 26,7 & $\mathrm{a}$ & 40,6 \\
\hline Máxima profundidade do defeito (d), mm & 2,95 & $\mathrm{a}$ & 9,65 \\
\hline $\mathrm{d} / \mathrm{t}$ & 0,31 & $\mathrm{a}$ & 1,0 \\
\hline Comprimento do defeito (2c), mm & 41,1 & $\mathrm{a}$ & 381,0 \\
\hline $2 \mathrm{c} /(\mathrm{Rt})^{0.5}$ & 0,683 & $\mathrm{a}$ & 7,752 \\
\hline Largura do defeito, mm & n.a & & \\
\hline Área exata do perfil do defeito, $\mathrm{mm}^{2}$ & 110,3 & $\mathrm{a}$ & 1478,7 \\
\hline Pressão de ruptura, $\mathrm{Nmm}^{-2}$ & 5,65 & $\mathrm{a}$ & 14,75 \\
\hline
\end{tabular}

\subsection{AGA/PRCI Database of Corroded Pipe Tests (1994)}

A base de dados de ensaio em dutos corroídos (AGA/PRCI) obteve resultados de ensaios em escala real (full-scale), que foram realizados por diferentes organizações para determinar os modos e tensões de falha de dutos corroídos. Vieth e Kiefner (1994) realizaram 124 ensaios em escala real em dutos retirados de serviço. A geometria de perda de metal é representada pela máxima profundidade, máximo comprimento do defeito e um perfil da área corroída ao longo do tubo.

Kiefner et al. (1995) realizaram 91 ensaios de ruptura, que foram adicionados à base de dados de Vieth e Kiefner (1994). Os parâmetros experimentais são apresentados na Tabela 3.2. 
Tabela 3.2. Parâmetros experimentais (Vieth; Kiefner, 1994).

\begin{tabular}{|c|c|c|c|}
\hline Diâmetro externo do duto (D), mm & 273,0 & $\mathrm{a}$ & 1219,2 \\
\hline Espessura da parede $(\mathrm{t}), \mathrm{mm}$ & 5,00 & $\mathrm{a}$ & 12,7 \\
\hline Relação 2R/t & 40,6 & $\mathrm{a}$ & 130,3 \\
\hline Grau (API 5L) & A & $\mathrm{a}$ & X65 \\
\hline Resistência ao escoamento (Y), $\mathrm{Nmm}^{-2} *$ & 196 & $\mathrm{a}$ & 515 \\
\hline Resistência à tração $(\mathrm{T}), \mathrm{Nmm}^{-2} *$ & 277 & $\mathrm{a}$ & 658 \\
\hline $\mathrm{Y} / \mathrm{T}$ & 0,58 & $\mathrm{a}$ & 0,93 \\
\hline 2/3 Energia de Impacto Charpy, J* & 18,0 & $\mathrm{a}$ & 96,7 \\
\hline Máxima profundidade do defeito (d), mm & 1,88 & $\mathrm{a}$ & 11,94 \\
\hline$d / t$ & 0,28 & $\mathrm{a}$ & 1,00 \\
\hline Comprimento do defeito (2c), $\mathrm{mm}$ & 19,35 & $\mathrm{a}$ & 3048,0 \\
\hline $2 \mathrm{c} /(\mathrm{Rt})^{0.5}$ & 0,527 & $\mathrm{a}$ & 49,7 \\
\hline Largura do defeito, mm* & 0,15 & & 762,0 \\
\hline Pressão de ruptura, $\mathrm{Nmm}^{-2}$ & 4,13 & $\mathrm{a}$ & 20,9 \\
\hline
\end{tabular}

\subsection{Battelle (1995)}

Stephens et al. (1995) realizaram dois ensaios de ruptura em corpos de prova com defeitos de corrosão artificial (usinados). No corpo de prova foi medida a deformação, pressão de falha e foram monitorados os volumes de água na pressurização do corpo de prova. Os defeitos artificiais foram criados por uma máquina conhecida como fresadora (Figura 3.1). A variação dos parâmetros experimentais encontra-se na Tabela 3.3. 

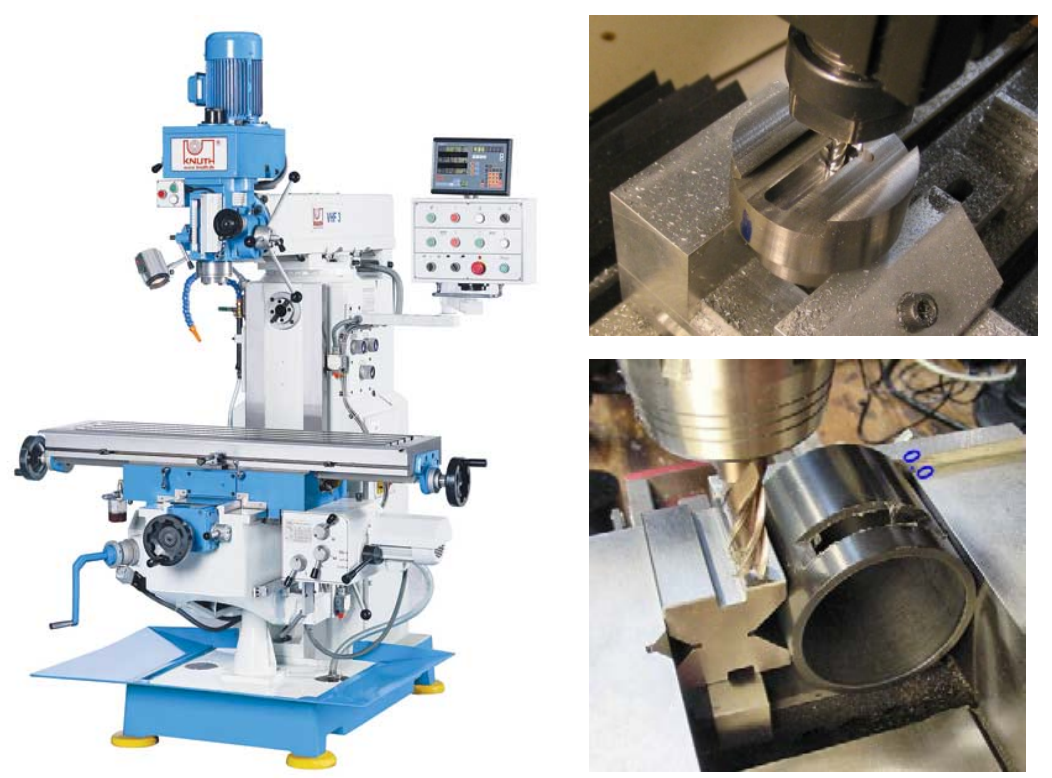

Figura 3.1. Máquina para realizar usinagem (fresadora).

Tabela 3.3. Parâmetros experimentais (Stephens et al., 1995).

\begin{tabular}{|c|c|c|c|}
\hline Diâmetro externo do duto (D), mm & 609,6 & & \\
\hline Espessura da parede (t), mm & 7,21 & & \\
\hline Relação 2R/t & 84,5 & & \\
\hline Grau (API 5L) & X52 & & \\
\hline Resistência ao escoamento (Y), $\mathrm{Nmm}^{-2}$ & 383,2 & & \\
\hline Resistência à tração $(\mathrm{T}), \mathrm{Nmm}^{-2}$ & 543,0 & & \\
\hline $\mathrm{Y} / \mathrm{T}$ & 0,71 & & \\
\hline 2/3 Energia de Impacto Charpy, $\mathrm{J}$ & 30,7 & & \\
\hline Máxima profundidade do defeito (d), mm & 2,38 & $\mathrm{a}$ & 4,32 \\
\hline $\mathrm{d} / \mathrm{t}$ & 0,33 & $\mathrm{a}$ & 0,60 \\
\hline Comprimento do defeito (2c), mm & 76,2 & $\mathrm{a}$ & 152,4 \\
\hline $2 \mathrm{c} /(\mathrm{Rt})^{0.5}$ & 1,63 & $\mathrm{a}$ & 3,25 \\
\hline Largura do defeito, mm & 38,1 & $\mathrm{a}$ & 304,8 \\
\hline Pressão de ruptura, $\mathrm{Nmm}^{-2}$ & 6,55 & $\mathrm{a}$ & 10,89 \\
\hline
\end{tabular}

\subsection{Petrobras (2000)}

Benjamin et al. (2000) realizaram 9 ensaios de ruptura em dutos de aço X60 de 323,9 mm de diâmetro e 9,53 mm de espessura de parede; que continha defeitos de corrosão externa 
artificial (usinados). Todos os corpos de prova utilizados nos ensaios tinham $2 \mathrm{~m}$ de comprimento. Também foram realizadas medições da espessura da parede nos corpos de prova ensaiados. Cada ensaio foi instrumentado com extensômetros de grande alongamento (strain gages). Os defeitos retangulares foram criados usando uma fresadora (Figura 3.1). Também foram monitoradas algumas características como a pressão interna e o volume de injeção de água no corpo de prova. A relação máxima da pressurização foi de 1.3 bar . min $^{-1}$. A variação dos parâmetros experimentais é apresentada na Tabela 3.4.

Tabela 3.4. Parâmetros experimentais (Benjamin et al., 2000).

\begin{tabular}{|c|c|c|c|}
\hline Diâmetro externo do duto (D), mm & 323,9 & & \\
\hline Espessura da parede (t), mm & 9,66 & $\mathrm{a}$ & 9,91 \\
\hline Relação 2R/t & 32,7 & $\mathrm{a}$ & 33,5 \\
\hline Grau (API 5L) & X60 & & \\
\hline Resistência ao escoamento (Y), $\mathrm{Nmm}^{-2}$ & 437,0 & $\mathrm{a}$ & 475,0 \\
\hline Resistência à tração $(\mathrm{T}), \mathrm{Nmm}^{-2}$ & 533,0 & $\mathrm{a}$ & 556,0 \\
\hline $\mathrm{Y} / \mathrm{T}$ & 0,83 & & \\
\hline 2/3 Energia de Impacto Charpy, J & n.a & & \\
\hline Máxima profundidade do defeito (d), mm & 6,53 & $\mathrm{a}$ & 6,89 \\
\hline $\mathrm{d} / \mathrm{t}$ & 0,67 & $\mathrm{a}$ & 0,70 \\
\hline Comprimento do defeito (2c), mm & 255,6 & $\mathrm{a}$ & 527,8 \\
\hline $2 \mathrm{c} /(\mathrm{Rt})^{0.5}$ & 6,42 & $\mathrm{a}$ & 13,3 \\
\hline Largura do defeito, mm & 95,3 & & \\
\hline Pressão de ruptura, $\mathrm{Nmm}^{-2}$ & 11,301 & $\mathrm{a}$ & 14,401 \\
\hline
\end{tabular}

\subsection{University of Waterloo (2000)}

Cronin e Pick (2000) realizaram 40 ensaios de ruptura em dutos que foram removidos de serviço, já que continham defeitos de corrosão (defeitos reais). As propriedades do material foram determinadas por ensaios a tração uniaxial em corpos de prova orientados em forma longitudinal e transversal. Um mínimo de quatro corpos de prova em ambas as direções foram ensaiados. Para obter uma informação detalhada dos defeitos foram utilizados scanners automáticos de alta qualidade (desenvolvidos pela Universidade de Waterloo) que realizaram 
medições com alta precisão do perfil corroído nas suas três dimensões. Além disso, foi realizado um mapeamento da área afetada. O diâmetro e a espessura da parede foi medida em quatro lugares do tubo, antes de se realizar o ensaio de ruptura. Os tubos foram fechados com tampas soldadas para formar corpos de prova para ensaio. Estes corpos de prova foram cheios e pressurizados com água a razão de $6 \mathrm{in}^{3} / \mathrm{min}$ até a ocorrer a falha. A falha era fotografada e o lugar de iniciação da falha era determinada baseando-se em: a localização do afunilamento da ligação, abaulamento do duto e da fratura da superfície. A variação dos parâmetros experimentais encontra-se na Tabela 3.5.

Tabela 3.5. Parâmetros experimentais (Cronin; Pick, 2000).

\begin{tabular}{|c|c|c|c|}
\hline Diâmetro externo do duto (D), mm & 273,0 & $\mathrm{a}$ & 863,6 \\
\hline Espessura da parede (t), mm & 4,57 & a & 9,63 \\
\hline Relação 2R/t & 33,1 & $\mathrm{a}$ & 95,5 \\
\hline Grau (API 5L) & $\mathrm{X} 42$ & $\mathrm{a}$ & X55 \\
\hline Resistência ao escoamento (Y), $\mathrm{Nmm}^{-2}$ & 350,6 & $\mathrm{a}$ & 462,3 \\
\hline Resistência à tração $(\mathrm{T}), \mathrm{Nmm}^{-2}$ & 453,9 & a & 587,3 \\
\hline $\mathrm{Y} / \mathrm{T}$ & 0,75 & $\mathrm{a}$ & 0,85 \\
\hline 2/3 Energia de Impacto Charpy, $\mathrm{J}$ & n.a & & \\
\hline Profundidade máxima do defeito (d), mm & 1,60 & a & 4,62 \\
\hline $\mathrm{d} / \mathrm{t}$ & 0,25 & a & 0,72 \\
\hline Comprimento do defeito (2c), $\mathrm{mm}$ & 30,5 & $\mathrm{a}$ & 1432,6 \\
\hline $2 \mathrm{c} /(\mathrm{Rt})^{0.5}$ & 1,19 & $\mathrm{a}$ & 32,29 \\
\hline Largura do defeito, mm & n.a & & \\
\hline Pressão de ruptura, $\mathrm{Nmm}^{-2}$ & 7,88 & $\mathrm{a}$ & 25,23 \\
\hline
\end{tabular}

\subsection{NOVA e Universidade de Waterloo (1990)}

Mok et al. (1990) realizaram duas series de ensaios totalizando 18 ensaios de ruptura em dutos de aço X60 de $508 \mathrm{~mm}$ de diâmetro, 6,35 mm e 6,40 mm de espessura da parede. Destes ensaios 12 continham defeitos de corrosão artificial que tinham orientação longitudinal e helicoidal, 6 ensaios continham reparações e defeitos longitudinal e circunferencial. As duas séries de ensaios encontram-se na Tabela 3.6. Também, foi realizado um detalhamento nas 
medições de espessura da parede, profundidade, largura e comprimento do defeito do corpo de prova, conforme Tabela 3.7.

Tabela 3.6. Resumo de ensaios realizados (Mok et al., 1990).

\begin{tabular}{|c|c|c|c|c|c|c|}
\hline $\begin{array}{c}\text { Coleta de } \\
\text { dados }\end{array}$ & $\begin{array}{c}\text { Número } \\
\text { de } \\
\text { ensaios }\end{array}$ & Grau & $\begin{array}{c}\mathbf{D} \\
(\mathrm{mm})\end{array}$ & $\begin{array}{c}\mathbf{t} \\
(\mathrm{mm})\end{array}$ & $\begin{array}{c}\mathbf{d} \\
(\mathrm{mm})\end{array}$ & Tipo de defeito \\
\hline \multirow{3}{*}{$1^{\text {era }}$ Serie } & 2 & \multirow{6}{*}{ X60 } & \multirow{6}{*}{508} & & \multirow{6}{*}{2,54} & Circunferencial \\
\hline & 8 & & & 6,35 & & $\begin{array}{l}\text { Longitudinal e } \\
\text { Helicoidal }\end{array}$ \\
\hline & 1 & & & & & Fissura paralela \\
\hline \multirow{3}{*}{$2^{\text {da }}$ Serie } & 3 & & & \multirow{3}{*}{6,4} & & Longitudinal \\
\hline & 2 & & & & & Circunferencial \\
\hline & 2 & & & & & Reparado \\
\hline
\end{tabular}

Tabela 3.7. Parâmetros experimentais (Mok et al., 1990).

Diâmetro externo do duto (D), mm

Espessura da parede (t), mm

Relação 2R/t

Grau (API 5L)

Resistência ao escoamento (Y), $\mathrm{Nmm}^{-2}$

Resistência à tração $(\mathrm{T}), \mathrm{Nmm}^{-2}$

$\mathrm{Y} / \mathrm{T}$

2/3 Energia de Impacto Charpy, J

Profundidade do defeito (d), mm

$\mathrm{d} / \mathrm{t}$

Comprimento longitudinal do defeito (2c), mm

$2 \mathrm{c} /(\mathrm{Rt})^{0.5}$

Comprimento circunferencial do defeito, $\mathrm{mm}$

Pressão de ruptura, $\mathrm{Nmm}^{-2}$
508,0

6,35 a $\quad 6,76$

75,1 a 79,4

X60

427,0 a 455,0

571,0 a 637,0

$0,69 \quad \mathrm{a} \quad 0,77$

56,7 a 96,7

2,18 a 3,45

0,34 a 0,54

25,4 a 1114,0

9,28 a 27,0

25,4 a 204,8

7,992 a 15,833 


\subsection{University of Waterloo (1992)}

Chouchaoui e Pick (1992) realizaram 12 ensaios de ruptura em um duto de aço X46 de 304,8 mm de diâmetro, 6,35 mm de espessura da parede, que continha corrosão interna por pites. Estes ensaios foram realizados em dutos que foram removidos de serviço por causa da corrosão. O duto esteve em serviço transportando petróleo bruto desde 1956. Dois dos corpos de prova ensaiados continham corrosão por pites, também foi considerado que a separação dos defeitos era adequada para trabalhar de forma independente; os corpos de prova restantes continham corrosão simples. A resistência ao escoamento nos corpos de prova ensaiados era grande, devido à anisotropia do material. Os parâmetros dos ensaios encontram-se na Tabela 3.8.

Tabela 3.8. Parâmetros experimentais (Chouchaoui; Pick, 1992).

\begin{tabular}{lcccc}
\hline Diâmetro do duto (D), mm & 304,8 & & \\
Espessura da parede (t), mm & 5,58 & a & 6,16 \\
Relação 2R/t & 49,5 & a & 54,6 \\
Grau (API 5L) & X46 & & \\
Resistência ao escoamento (Y), Nmm ${ }^{-2}$ & 346,0 & a & 421,0 \\
Resistência à tração (T), Nmm ${ }^{-2}$ & 441,2 & a & 512,7 \\
Y/T & 0,68 & a & 0,85 \\
2/3 Energia de Impacto Charpy, J & n.a & & \\
Profundidade do defeito por pit (d), mm & 1,99 & a & 4,68 \\
d/t & 0,33 & a & 0,79 \\
Comprimento do defeito por pit (2c), mm & 26,0 & a & 59,0 \\
2c/(Rt) & 0,5 & a & 1,94 \\
Pressão de ruptura, Nmm ${ }^{-2}$ & 4,13 & a & 16,95 \\
\hline
\end{tabular}

\subsection{University of Waterloo (1994-1996)}

Chouchaoui e Pick (1994) realizaram 2 conjuntos de ensaios totalizando 14 ensaios de ruptura em dutos de aço X46 de 304,8 mm de diâmetro, 6,35 mm de espessura da parede, que continham defeitos de corrosão artificial por pites. O primeiro conjunto continha 6 ensaios, 
onde os defeitos tinham fundo plano e uma forma circular de diâmetro 20,9; 55,5 ou 106,9 $\mathrm{mm}$.

Estes defeitos apresentavam as seguintes características: (1) um pit pequeno no meio da área corroída. (2) um pit deslocado em uma direção longitudinal ou circunferencial. Os pites de menor profundidade eram de 39\% da espessura da parede e os de maior profundidade eram de $67 \%$ da espessura da parede. O segundo conjunto continha 8 ensaios onde os defeitos de corrosão por pites eram alinhados em forma circunferencial. Estes defeitos continham fundo plano e a profundidade era o 59\% da espessura da parede, sua forma era em forma elíptica com um diâmetro maior de 19,90 mm e um menor de 19,71 mm aproximadamente. Um terceiro conjunto foi realizado por Chouchaoui e Pick (1996), onde se obtiveram 8 ensaios de ruptura em um duto com as mesmas características assumidas por Chouchaoui e Pick (1994). Estes defeitos continham fundo plano e a profundidade era de $58 \%$ da espessura da parede, também eram de forma elíptica com um diâmetro menor de 19,98 mm e um maior de 20,81 mm aproximadamente. Os defeitos dos três conjuntos foram criados por uma fresadora (Figura 3.1). A variação dos parâmetros experimentais obtidos por Chouchaoui e Pick (1994) encontra-se na Tabela 3.9 e Tabela 3.10 e para Chouchaoui e Pick (1996) encontra-se na Tabela 3.11.

Tabela 3.9. Parâmetros experimentais para o primeiro conjunto (Chouchaoui; Pick, 1994).

\begin{tabular}{lccc}
\hline Diâmetro do duto (D), mm & 324,0 & a & 325,0 \\
Espessura da parede (t), mm & 6,59 & a & 6,85 \\
Relação 2R/t & 47,4 & a & 49,3 \\
Grau (API 5L) & X46 & & \\
Resistência ao escoamento (Y), Nmm ${ }^{-2}$ & 359,0 & a & 403,0 \\
Resistência à tração (T), Nmm ${ }^{-2}$ & 470,3 & a & 508,9 \\
Y/T & 0,75 & a & 0,81 \\
2/3 Energia de Impacto Charpy, J & n.a & & \\
Profundidade do pit (d), mm & 4,22 & a & 4,64 \\
d/t & 0,64 & a & 0,69 \\
Comprimento do defeito (2c), mm & 56,0 & a & 108,0 \\
2c/(Rt) & 1,68 & a & 3,27 \\
Pressão de ruptura, Nmm ${ }^{-2}$ & 13,98 & a & 16,50 \\
\hline
\end{tabular}


Tabela 3.10. Parâmetros experimentais para o segundo conjunto (Chouchaoui; Pick, 1994).

\begin{tabular}{lccc}
\hline Diâmetro do duto (D), mm & 323,0 & a & 324,0 \\
Espessura da parede (t), mm & 6,01 & a & 6,40 \\
Relação 2R/t & 50,6 & a & 53,9 \\
Grau (API 5L) & X46 & & \\
Resistência ao escoamento (Y), Nmm ${ }^{-2}$ & 356,0 & a & 421,0 \\
Resistência à tração (T), Nmm ${ }^{-2}$ & 451,5 & a & 496,8 \\
Y/T & 0,77 & a & 0,93 \\
2/3 Energia de Impacto Charpy , J & n.a & & \\
Profundidade do pit (d), mm & 3,23 & a & 3,79 \\
d/t & 0,50 & a & 0,61 \\
Comprimento do defeito (2c), mm & 19,35 & a & 20,1 \\
2c/(Rt) & 0,620 & a & 0,637 \\
Pressão de ruptura, Nmm ${ }^{-2}$ & 14,16 & a & 19,44 \\
\hline
\end{tabular}

Tabela 3.11. Parâmetros experimentais para o terceiro conjunto (Chouchaoui; Pick, 1996).

\begin{tabular}{|c|c|c|c|}
\hline Diâmetro do duto (D), mm & 322,0 & $\mathrm{a}$ & 325,0 \\
\hline Espessura da parede (t), mm & 6,16 & $\mathrm{a}$ & 6,45 \\
\hline Relação 2R/t & 50,4 & $\mathrm{a}$ & 56,6 \\
\hline Grau (API 5L) & $\mathrm{X} 46$ & & \\
\hline Resistência ao escoamento (Y), $\mathrm{Nmm}^{-2}$ & 356,0 & a & 381,0 \\
\hline Resistência à tração $(\mathrm{T}), \mathrm{Nmm}^{-2}$ & 447,5 & $\mathrm{a}$ & 470,4 \\
\hline $\mathrm{Y} / \mathrm{T}$ & 0,79 & a & 0,82 \\
\hline 2/3 Energia de Impacto Charpy , J & n.a & & \\
\hline Profundidade do pit (d), mm & 3,05 & $\mathrm{a}$ & 3,79 \\
\hline $\mathrm{d} / \mathrm{t}$ & 0,47 & $\mathrm{a}$ & 0,61 \\
\hline Comprimento do defeito (2c), mm & 19,69 & $\mathrm{a}$ & 20,82 \\
\hline $2 \mathrm{c} /(\mathrm{Rt})^{0.5}$ & 0,610 & $\mathrm{a}$ & 0,643 \\
\hline Pressão de ruptura, $\mathrm{Nmm}^{-2}$ & 13,87 & $\mathrm{a}$ & 17,61 \\
\hline
\end{tabular}




\subsection{University of Waterloo (1998)}

Roberts e Pick (1998) realizaram 10 ensaios de ruptura em dutos de aço X42 de 323,85 mm de diâmetro e 9,65 mm de espessura da parede, que continham defeitos de corrosão artificial por pites. Os corpos de prova eram de $6 \mathrm{ft}$ de comprimento especialmente projetados para ensaios a tração ou compressão. Os defeitos de corrosão artificiais foram criados por uma fresadora (Figura 3.1). Os parâmetros experimentais são apresentados na Tabela 3.12.

Tabela 3.12. Parâmetros experimentais (Roberts; Pick, 1998).

\begin{tabular}{|c|c|c|c|}
\hline Diâmetro do duto (D), mm & 323,9 & & \\
\hline Espessura da parede $(\mathrm{t}), \mathrm{mm}$ & 9,65 & & \\
\hline Relação 2R/t & 33,6 & & \\
\hline Grau (API 5L) & $\mathrm{X} 42$ & & \\
\hline Resistência ao escoamento (Y), $\mathrm{Nmm}^{-2}$ & 334,4 & & \\
\hline Resistência à tração $(\mathrm{T}), \mathrm{Nmm}^{-2^{*}}$ & 571,6 & & \\
\hline $\mathrm{Y} / \mathrm{T}$ & 0,59 & & \\
\hline 2/3 Energia de Impacto Charpy, $\mathrm{J}$ & n.a & & \\
\hline Profundidade do defeito (d), mm & 5,69 & $\mathrm{a}$ & 8,53 \\
\hline $\mathrm{d} / \mathrm{t}$ & 0,59 & $\mathrm{a}$ & 0,88 \\
\hline Comprimento do defeito (2c), mm & 30,5 & $\mathrm{a}$ & 35,6 \\
\hline $2 \mathrm{c} /(\mathrm{Rt})^{0.5}$ & 0,771 & a & 0,899 \\
\hline Pressão de ruptura, $\mathrm{Nmm}^{-2}$ & 26,5 & $\mathrm{a}$ & 29,2 \\
\hline
\end{tabular}

\subsection{Transgas (2000)}

Nehoda e Horalek (2000) realizaram 17 ensaios de ruptura em dutos com diferentes geometrias contendo defeitos por corrosão artificial nas paredes dos corpos de prova, defeitos de corrosão real, fissuras e laminações. A variação dos parâmetros experimentais encontra-se na Tabela 3.13. 
Tabela 3.13. Parâmetros experimentais (Nehoda; Horalek, 2000).

\begin{tabular}{|c|c|c|c|}
\hline Diâmetro do duto (D), mm & 325,0 & $\mathrm{a}$ & 1020,0 \\
\hline Espessura da parede (t), mm & 8,0 & $\mathrm{a}$ & 16,0 \\
\hline Relação 2R/t & 40,6 & $\mathrm{a}$ & 89,5 \\
\hline Grau (API 5L) & $\mathrm{X} 60$ & $\mathrm{a}$ & $\mathrm{X} 70$ \\
\hline Resistência ao escoamento (Y), $\mathrm{Nmm}^{-2}$ & n.a & & \\
\hline Resistência à tração $(\mathrm{T}), \mathrm{Nmm}^{-2^{*}}$ & n.a & & \\
\hline 2/3 Energia de Impacto Charpy, J & n.a & & \\
\hline Profundidade do defeito (d), mm & 3,3 & $\mathrm{a}$ & 10,0 \\
\hline Relação d/t & 0,29 & $\mathrm{a}$ & 0,68 \\
\hline Comprimento do defeito (2c), mm & 115,0 & $\mathrm{a}$ & 790,0 \\
\hline $2 \mathrm{c} /(\mathrm{Rt})^{0.5}$ & 1,69 & $\mathrm{a}$ & 11,8 \\
\hline Pressão de ruptura, $\mathrm{Nmm}^{-2}$ & 8,10 & $\mathrm{a}$ & 27,7 \\
\hline
\end{tabular}

\subsection{Det Norske Veritas (2000)}

Bjørnoy et al. (2000) realizaram 2 ensaios experimentais em um duto de aço X52 de 324 mm de diâmetro nominal, 10,3 de espessura da parede que continha defeitos de corrosão artificial externa sujeito a pressão interna. Os defeitos de corrosão longitudinal foram criados por uma fresadora (Figura 3.1). Os parâmetros são encontrados na Tabela 3.14.

\subsection{British Gas (1999) (Linepipe Corrosion Group Sponsored Project)}

Fu e Batte (1999) realizaram 81 ensaios de ruptura e 52 ensaios de expansão do anel em dutos com defeitos de corrosão artificial sujeito a pressão interna. Também, incluído 6 ensaios de ruptura e 16 ensaios do anel que foram fornecidos pela sponsors of the project. Na Tabela 3.15 encontra-se a variação dos parâmetros experimentais. 
Tabela 3.14. Parâmetros experimentais (Bjørnoy et al., 2000)

\begin{tabular}{lcccc}
\hline Diâmetro do duto (D), mm & 324,0 & & \\
Espessura da parede (t), mm & 10,25 & a & 11,10 \\
Relação 2R/t & 29,2 & a & 31,6 \\
Grau (API 5L) & X52 & & \\
Resistência ao escoamento (Y), Nmm ${ }^{-2}$ & 380,0 & & \\
Resistência à tração (T), Nmm ${ }^{-2}$ & 514,0 & & \\
Y/T & 0,74 & & \\
2/3 Energia de Impacto Charpy, J & 69,0 & & \\
Profundidade do defeito (d), mm & n.a & & \\
d/t & 0,30 & a & 0,70 \\
Comprimento do defeito (2c), mm & 12,0 & a & 243,0 \\
2c/(Rt) & 0.5 & 0,294 & a & 5,95 \\
Pressão de ruptura, Nmm & \\
\hline
\end{tabular}

Tabela 3.15. Parâmetros experimentais (Fu; Batte, 1999)

\begin{tabular}{lccc}
\hline Diâmetro do duto (D), mm & 219,2 & & 914,4 \\
Espessura da parede (t), mm & 9,5 & a & 22,5 \\
Relação 2R/t & 8,6 & a & 47,6 \\
Grau (API 5L) & X52 & a & X65 \\
Resistência ao escoamento (Y), Nmm ${ }^{-2}$ & n.a & & \\
Resistência à tração (T), Nmm & n.a & & \\
Y/T & n.a & & \\
2/3 Energia de Impacto Charpy, J & n.a & & \\
Profundidade do defeito (d), mm & 5,08 & a & 22,86 \\
d/t & 0,20 & a & 0,90 \\
Comprimento do defeito (2c), mm & 19,0 & a & 812,8 \\
2c/(Rt) & n.5 & & \\
Pressão de ruptura, Nmm & \\
\hline
\end{tabular}

É importante lembrar que a base de dados pode ser encontrada no documento "The Pipeline

Defect Assessment Manual Joint Industry Project” (Cosham, 2002). 


\section{INFERÊNCIA ESTATÍSTICA}

\subsection{Variável Aleatória}

Uma variável aleatória real é uma função que associa um valor real a cada ponto de um espaço amostral. Uma variável aleatória pode adquirir qualquer valor definido por um intervalo, tal que o evento $\{\boldsymbol{X}=x\}$ indica que a variável aleatória $\boldsymbol{X}$ assume o valor de $x$. O evento $\{\boldsymbol{X} \leq x\}$ indica que a variável aleatória $\boldsymbol{X}$ assume qualquer valor menor ou igual do que $x$. Cabe destacar que uma variável aleatória é representada por uma letra maiúscula e uma realização é representada por uma letra minúscula.

O espaço amostral $\Omega$ é o domínio da variável aleatória, mas dependendo desse espaço amostral $\Omega$ a variável aleatória pode ser do tipo discreta ou contínua. Ela é discreta, quando $\Omega$ é formado por um número finito ou infinito contável de pontos amostrais, e contínua quando o $\Omega$ é formado por um numero infinito de pontos. Pode-se dizer que uma variável aleatória real $\boldsymbol{X}(\omega)$ é uma função real que atribui a cada ponto amostral $\omega$ de um espaço amostral $\Omega$ um valor real $x$, tal que o conjunto $\{\boldsymbol{X} \leq x\}$ é um evento para qualquer número real $x$. Nestes casos pode ser observado que o espaço amostral $\Omega$ é o domínio de $\boldsymbol{X}(\omega)$ (Beck, 2012).

\subsubsection{Função de distribuição acumulada de probabilidade}

Quando se quer conhecer a probabilidade de ocorrência de um conjunto definido por $\{\boldsymbol{X} \leq x\}$; a probabilidade deste evento depende apenas de $x$, que nos leva à existência da função $F_{X}(x)=P[\{X \leq x\}]$. Essa função é considerada como função de distribuição acumulada de probabilidades (FDA) da variável aleatória $\boldsymbol{X}$, definida para o intervalo $-\infty \leq x \leq+\infty$. 
A Equação (4.1) segundo Montgomery e Runger (2003) define a função de distribuição acumulada de uma variável aleatória $\boldsymbol{X}$ como:

$F_{X}(x)=P[\{X \leq x\}]=\int_{-\infty}^{x} f_{X}(x) d x$,

onde $f_{X}(x)$ é a função de densidade de probabilidade da variável aleatória $\boldsymbol{X}$, que será apresentada na seguinte seção.

\subsubsection{Função de densidade de probabilidade}

A derivada da função acumulada de probabilidade em relação a $x$ define a função de densidade de probabilidade (FDP) de $\boldsymbol{x}$, que é $f_{X}(x)$, sendo obtida como:

$f_{X}(x)=\frac{d F_{X}(x)}{d x}$

A função de densidade de probabilidade é usada para descrever uma variável aleatória, já que ela pode ser discreta ou contínua. Da teoria de Probabilidade (Montgomery e Runger, 2003) sabe-se que $f_{X}(x)$ é uma função que satisfaz as seguintes condições:

$$
\begin{aligned}
& f_{X}(x) \geq 0 ; \\
& \int_{-\infty}^{\infty} f_{X}(x) d x=1 ; \\
& P[\{a \leq X \leq b\}]=\int_{a}^{b} f_{X}(x) d x .
\end{aligned}
$$

\subsubsection{Momentos de uma variável aleatória}

Para conseguir caracterizar uma variável aleatória $\boldsymbol{X}$ a partir da função de densidade de probabilidade é necessário definir os momentos. O momento de ordem $k$ para uma variável aleatória contínua se define como: 
$E\left[X^{k}\right]=\mu^{k}=\int_{-\infty}^{+\infty} x^{k} f_{X}(x) d x$

A generalização do valor esperado de $\boldsymbol{X}$, sendo um momento de primeira ordem é obtido como:

$E[X]=\mu=\int_{-\infty}^{+\infty} x f_{X}(x) d x$

onde a média ou valor esperado de $\boldsymbol{X}$, são denotados por $\mu$ ou $E[$.].

Os momentos centrais de ordem $k$ para variáveis aleatórias contínuas são obtidos em relação à média $\mu$, conforme a seguinte expressão:

$E\left[(X-\mu)^{k}\right]=m^{k}=\int_{-\infty}^{+\infty}(x-\mu)^{k} f_{X}(x) d x$

A variância se representa como o momento central de segunda ordem como:

$E\left[(X-\mu)^{2}\right]=m^{2}=\int_{-\infty}^{+\infty}(x-\mu)^{2} f_{X}(x) d x$.

Dessa maneira, a variância também pode ser escrita da seguinte forma:

$\operatorname{Var}[X]=E\left[(X-\mu)^{2}\right]=\sigma^{2}$.

A variância e desvio padrão medem a dispersão da variável em torno da média, de tal forma que $\sigma=\sqrt{\operatorname{Var}[X]}$, sendo $\sigma$ o desvio padrão. 


\subsubsection{Média e Variância de amostras}

Quando é tomada uma amostra de tamanho $n$ de uma população os parâmetros da amostra como a média $\bar{\mu}$ e a variância $\bar{\sigma}^{2}$ podem ser determinadas como:

$\bar{\mu}=\frac{1}{n} \sum_{i=1}^{n} x_{i}$

$\bar{\sigma}^{2}=\frac{1}{n-1} \sum_{i=1}^{n}\left(x_{i}-\bar{\mu}\right)^{2}$

Neste caso, tem-se que a variância apresenta uma divisão pelo termo $n-1$. Isto acontece porque a variância esta sendo medida pela distância dos pontos $x_{i}$ em relação média da amostra $\bar{\mu}$, e não em relação à média populacional $\mu$. Portanto, para corrigir a tendenciosidade é utilizada a Equação (4.12).

\subsubsection{Distribuição de Probabilidade Normal ou Gaussiana}

Uma variável aleatória $\boldsymbol{X}$ normalmente distribuída ou gaussiana é simétrica, possuindo dois parâmetros que são os próprios momentos, média $(\mu)$ e desvio padrão $(\sigma)$. A função de distribuição de probabilidade (FDP) para a distribuição normal é dada por:

$$
f_{X}(x)=\frac{1}{\sigma \sqrt{2 \pi}} \exp \left[\frac{-1}{2}\left(\frac{x-\mu}{\sigma}\right)^{2}\right] \quad-\infty \leq x \leq \infty \text {. }
$$

A função de distribuição cumulativa de probabilidade é representada como:

$$
f_{X}(x)=\int_{-\infty}^{x} \frac{1}{\sigma \sqrt{2 \pi}} \exp \left[\frac{-1}{2}\left(\frac{z-\mu}{\sigma}\right)^{2}\right] d z
$$


A função cumulativa de probabilidade só pode ser avaliada por integração numérica, já que não possui solução analítica; geralmente ela é resolvida por tabelas encontradas na literatura ou por análise numérica.

Por outro lado, existe uma alternativa equivalente, onde são apresentadas as funções em termos de uma distribuição normal com média nula $(\mu=0)$ e desvio padrão unitário $(\sigma=1)$, bem conhecido, como distribuição normal padrão. Portanto, qualquer variável aleatória que possua a forma $X \sim N(\mu, \sigma)$ pode ser transformada em variável normal padrão $Y$ da forma:

$Y=\frac{X-\mu}{\sigma}$

A partir das Equações (4.16) e (4.17) obtém-se as funções de probabilidades, dada por:

$$
\begin{array}{ll}
f_{Y}(y)=\phi(y)=\frac{1}{\sqrt{2 \pi}} \exp \left[-\frac{y^{2}}{2}\right] & -\infty \leq x \leq \infty ; \\
F_{Y}(y)=\int_{-\infty}^{y} \phi(z) d z & -\infty \leq x \leq \infty,
\end{array}
$$

onde $\phi($.) é a função cumulativa normal padrão. A função de densidade normal padrão pode ser vista na Figura 4.1.

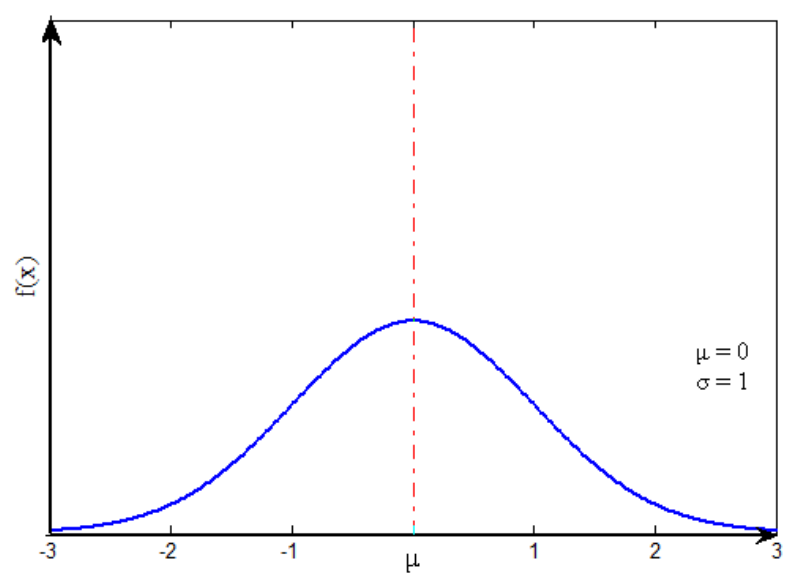

Figura 4.1. Função de densidade de probabilidade normal padrão. 


\subsubsection{Distribuição log-normal $\operatorname{LN}(\lambda, \xi)$}

Uma variável $\boldsymbol{X}$ possui distribuição log-normal, quando o $\ln (x)$ segue uma distribuição normal. Tais funções de probabilidade são dadas por:

$$
\begin{aligned}
& f_{X}(x)=\frac{1}{\xi x \sqrt{2 \pi}} \exp \left[-\frac{1}{2}\left(\frac{\ln (x)-\lambda}{\xi}\right)\right] \quad 0 \leq x \leq \infty, \\
& F_{X}(x)=\Phi\left[\frac{\ln (x)-\lambda}{\xi}\right] \quad 0 \leq x \leq \infty,
\end{aligned}
$$

sendo $\lambda$ o valor esperado de $\ln (x), \xi$ o desvio padrão. Estes parâmetros são obtidos a partir dos momentos, por:

$$
\begin{aligned}
& \lambda=\ln (\mu)-0.5 \xi^{2} ; \\
& \xi=\sqrt{\ln \left(1+(\sigma / \mu)^{2}\right)} .
\end{aligned}
$$

Os momentos de uma variável log-normal são obtidos a partir dos parâmetros por:

$$
\begin{aligned}
& \mu=\exp \left[\lambda+0.5 \xi^{2}\right] \\
& \sigma=\mu \sqrt{\exp \left[\xi^{2}\right]-1}
\end{aligned}
$$

Na análise de confiabilidade é importante conhecer os parâmetros da distribuição equivalente, obtidos a partir das expressões a seguir:

$$
\begin{aligned}
& \mu^{\text {neq }}=x(1-\ln (x)+\lambda) ; \\
& \sigma^{\text {neq }}=x \xi .
\end{aligned}
$$




\subsubsection{Gumbel para máximos ou tipo I - EVI $\left(u_{n}, \beta\right)$}

A distribuição de valor extremo ou Gumbel surge quando se toma o logaritmo natural de uma variável que apresente distribuição Weibull. Se a variável $x$ apresenta distribuição Weibull

com parâmetros $u_{n}$ (escala) e $\beta$ (forma) então a variável $X=\ln (x)$ tem uma distribuição Gumbel, com funções de probabilidades:

$$
\begin{aligned}
& f X_{n}(x)=\beta \exp \left[-\beta\left(x-u_{n}\right)-e^{-\beta\left(x-u_{n}\right)}\right] \quad-\infty \leq x \leq \infty, \\
& F X_{n}(x)=\exp \left[-e^{-\beta\left(x-u_{n}\right)}\right] \quad-\infty \leq x \leq \infty,
\end{aligned}
$$

sendo, os parâmetros de distribuição os seguintes:

$$
\begin{aligned}
& u_{n}=\mu-\frac{\gamma}{\beta}, \\
& \beta=\frac{\pi}{\sqrt{6}} \frac{1}{\sigma} .
\end{aligned}
$$

A média e a variância da variável aleatória $\boldsymbol{X}$ são dadas por:

$$
\begin{aligned}
& \mu=u_{n}+\frac{\gamma}{\beta} \\
& \sigma=\frac{\pi}{\sqrt{6}} \frac{1}{\beta}
\end{aligned}
$$

onde: $\gamma=0,577216$ (constante de Euler). 


\subsection{Testes de hipótese}

\subsubsection{Teste de hipótese de Kolmogorov-Smirnov}

Os métodos estatísticos tradicionais são baseados em suposições paramétricas. Isto se deve, ao fato que os dados proveem da mesma família paramétrica (Normal, exponencial, etc.). Portanto, alguns fenômenos não necessariamente podem ser atribuídos a uma distribuição conhecida. O método não paramétrico não é baseado em famílias com parâmetros de distribuição de probabilidade. Este método não faz suposições das distribuições de probabilidade das variáveis que estão sendo avaliadas.

O teste de Kolmogorov-Smirnov é um teste de aderência não paramétrico que mede o grau de concordância existente entre a distribuição de um conjunto de dados de determinada distribuição teórica específica. O teste é usado para saber se os valores amostrais podem provavelmente ser considerados de uma mesma população com uma suposta distribuição teórica. O teste é uma poderosa ferramenta para verificar a aderência de grandes amostras, admitindo que a distribuição da variável testada seja contínua, já que os valores críticos para distribuições discretas não são encontrados em tabelas. Mediante o teste, ou seja, comparando a distribuição de frequência acumulada teórica (FDA) com a distribuição de frequência acumulada observada, se determina o ponto de maior divergência entre as distribuições.

Seja $F_{X}$ a função de distribuição de frequências acumulada contínua de uma população, e seja $F_{0}(X)$ a função específica de distribuição de frequência acumulada teórica representada como:

$F_{0}\left(x_{i}\right)=\mathrm{P}\left(X \leq x_{i}\right)$,

onde $F_{n}$ é a distribuição de frequências observada acumulada de uma amostra aleatória $X_{1}, X_{2}, \ldots, X_{n}$ de $n$ observações. Para o teste é necessário uma distribuição de frequência acumulada observada que pode ser obtida como:

$$
F_{n}(x)=\frac{1}{n} \sum_{i=1}^{n} I_{\{(-\infty, x]\}}\left(x_{i}\right),
$$


onde $I_{A}$ é a função indicadora que é representada por:

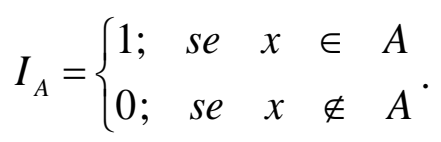

As hipóteses utilizadas pelo teste são consideradas a partir de:

$\mathrm{H}_{0}$ : A população de onde provêm as amostras segue uma distribuição especifica $\left[F_{X}=F_{0}\right.$ para algum $\left.x\right]$.

$\mathrm{H}_{1}$ : A população de onde provêm as amostras não segue uma distribuição especifica $\left[F_{X} \neq F_{0}\right.$ para algum $\left.x\right]$.

No teste Kolmogorov-Smirnov compara-se as distribuições de frequência relativa acumulada da amostra e a distribuição de frequência acumulada teórica, que se esperaria que satisfizesse a hipótese nula. No teste se considera a maior das diferenças das distribuições e verifica-se a normalidade da amostra podendo ser obtida através de:

$D=\max \left|F_{0}(x)-F_{n}(x)\right|$,

onde $D$ representa a distância máxima vertical (Figura 4.2), entre a comparação das duas distribuições $F_{0}(x)$ e $F_{n}(x)$.

\subsubsection{Teste de hipótese de Anderson-Darling (A-D)}

O teste de hipótese Anderson-Darling é utilizado para confirmar, se um conjunto de dados pertence a uma mesma população com uma distribuição específica. O teste A-D é uma modificação do teste Kolmogorov-Smirnov (teste K-S), pois dá mais peso as caudas em relação ao teste K-S. 


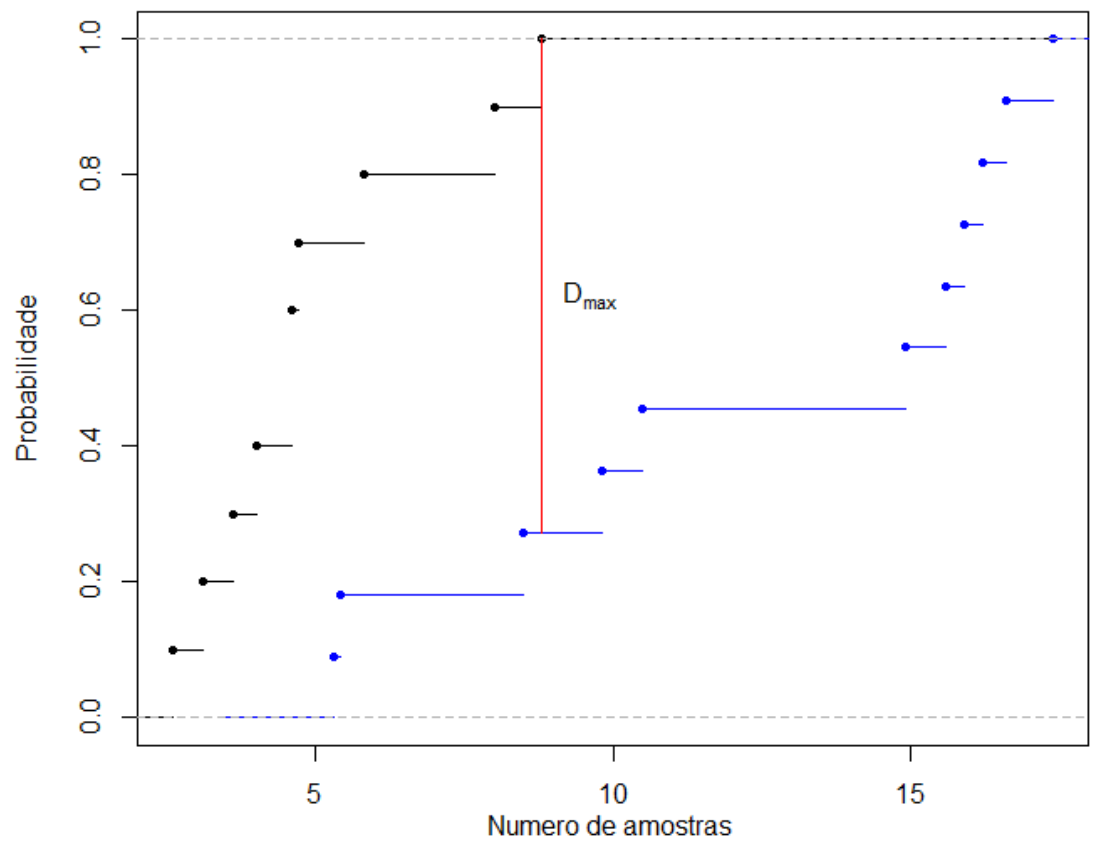

Figura 4.2. Ajustamento de uma função de distribuição hipotética $F$ à função de distribuição empírica $F_{n}$.

Ao se comparar com o teste apresentado anteriormente (teste K-S), pode-se dizer que é um teste que não depende dos valores críticos da distribuição específica que esta sendo testada. Porém, o teste A-D utiliza valores críticos calculados a partir de uma distribuição específica. Com essas características tem-se uma vantagem de ter maior sensibilidade, mas também possui a desvantagem, os valores críticos sempre devem ser calculados para cada tipo de distribuição. O teste de hipótese verifica a adequabilidade da distribuição às hipóteses (Equação (4.29)). O teste A-D é definido como:

$$
A^{2}=n \int_{-\infty}^{\infty} \frac{\left[F_{n}(x)-F_{0}(x)\right]^{2}}{\left[F_{0}(x)\left(1-F_{0}(x)\right)\right]} d F_{0}(x),
$$

onde $F_{n}$ é a função empírica acumulada e $F_{0}$ é a função específica de distribuição de frequência acumulada teórica escolhida. A Equação (4.31) pode ser escrita em uma forma equivalente, dada por:

$$
A_{n}^{2}=-n-\frac{1}{n} \sum_{i=1}^{n} S_{i}
$$


onde $S_{i}$ é uma função obtida a partir de:

$S_{i}=(2 i-1)\left[\ln F\left(x_{i}\right)+\ln \left(1-F\left(x_{N+1-i}\right)\right)\right]$,

sendo $\mathrm{F}$ a distribuição cumulativa dos dados. Cabe lembrar que os valores da amostra $x_{i}$ devem encontrar-se em ordem crescente.

\subsubsection{Teste de hipótese Qui-quadrado}

O teste de hipótese qui-quadrado $\left(\chi^{2}\right)$ é um teste de aderência considerado não paramétrico associado entre variáveis, cujas observações são discretas. No teste, verifica-se a existência de discrepância entre a distribuição de frequências observadas e a distribuição das frequências teóricas. O teste é utilizado para comprovar se duas variáveis aleatórias estão correlacionadas entre si. O modelo estatístico do teste é dado por:

$\chi^{2}=\sum_{i=1} \frac{\left(F_{n}-F_{0}\right)^{2}}{F_{0}}$.

Esta expressão indica a semelhança existente entre as distribuições, sendo necessário ter um critério de avaliação da hipótese:

- Se $\chi^{2} \leq m$, quer dizer que a hipótese é aceita e as distribuições são da mesma população.

- Se $\chi^{2}>>m$, é bem provável que a hipótese seja rejeitada, indicando que as distribuições são de populações diferentes.

\subsection{Regressão Linear e Não-Linear}

\subsubsection{Regressão Linear}

A regressão linear é uma modelagem estatística utilizada para estudar a relação entre duas ou mais variáveis, onde estas variáveis são consideradas como: variável independente e variável de resposta, podendo ser definidas a partir de outras variáveis envolvidas no problema. 
Quando as variáveis são relacionadas, pode-se representar por um modelo matemático que associa as variáveis dependentes com as independentes. O modelo linear probabilístico utilizado pode ser representado por:

$Y=\beta_{0}+\beta_{1} \cdot x+\varepsilon$,

onde $Y$ é a variável de resposta ou variável dependente, $x$ é variável explicativa ou variável independente, e $\beta$ representa os coeficientes de regressão, sendo $\beta_{0}$ uma constante que representa a interseção da reta com o eixo vertical, e $\beta_{1}$ a constante que representa a inclinação da reta. O termo $\varepsilon$ inclui todos os fatores residuais mais os possíveis erros de medição. Ela é uma variável com um comportamento aleatório. O erro aleatório possui uma distribuição normal com média nula $(E[\varepsilon]=0)$ e variância constante que deve ser determinada $\left(\operatorname{Var}[\varepsilon]=\sigma^{2}\right)$.

Para obter os coeficientes de regressão pode ser utilizado o método dos mínimos quadrados (Figura 4.3). Este método realiza uma técnica de optimização que minimiza a soma dos quadrados, resultando a mínima diferença entre o valor estimado e as observações.

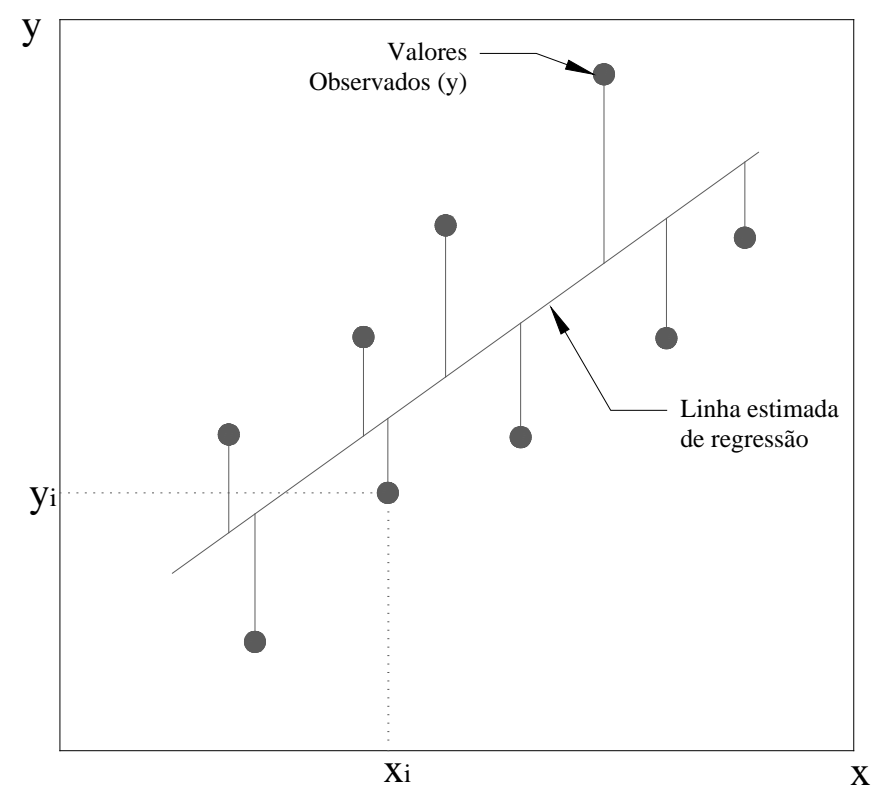

Figura 4.3. Desvio dos dados em relação ao modelo estimado de regressão [Montgomery e Runger, 2003 - adaptado]. 
Portanto, o modelo de regressão ajustado pode ser representado como:

$\hat{y}_{i}=\hat{\beta}_{0}+\hat{\beta}_{1} \cdot x_{i}+e_{i}$,

onde $\hat{\beta}_{0}$ uma estimativa da interseção obtida pelo método dos mínimos quadrados, o qual é obtido por:

$\beta_{0}=\bar{y}-\hat{\beta}_{1} \cdot \bar{x}$.

Portanto, as médias amostrais de $x$ e $y$, podem ser calculadas como:

$\bar{y}=\frac{1}{n} \sum_{i=1}^{n} y_{i}$

$\bar{x}=\frac{1}{n} \sum_{i=1}^{n} x_{i}$

O coeficiente que mede a inclinação do modelo de regressão é obtido pelo método dos mínimos quadrados, dado por:

$\hat{\beta}_{1}=\frac{\sum_{i=1}^{n} y_{i} x_{i}-\frac{\left(\sum_{i=1}^{n} y_{i}\right)\left(\sum_{i=1}^{n} x_{i}\right)}{n}}{\sum_{i=1}^{n} x_{i}^{2}-\frac{\left(\sum_{i=1}^{n} x_{i}\right)^{2}}{n}}$.

Entretanto, os resíduos estimados a partir do ajuste são avaliados por:

$e_{i}=\hat{y}_{i}-\hat{y}_{i}$. 


\subsubsection{Regressão não-linear}

Às vezes é muito importante a utilização de modelos de regressão não-linear, já que em algumas situações não é possível descrever um fenômeno através de um modelo de regressão linear. Um método de regressão é não linear, quando pelo menos um dos seus parâmetros ou a variável dependente não pode ser representado em forma linear. Estes modelos não lineares tem uma vantagem porque os parâmetros obtidos podem ser facilmente interpretáveis. Enfim, os modelos não lineares se representam como:

$Y_{i}=f\left(x_{i}, \gamma\right)+\varepsilon_{i}$,

sendo que as variáveis envolvidas se representam em forma matricial como:

$$
\underset{q \times 1}{x_{i}}=\left[\begin{array}{c}
x_{i 1} \\
x_{i 2} \\
\vdots \\
x_{i q}
\end{array}\right] \quad \underset{p \times 1}{\gamma}=\left[\begin{array}{c}
\gamma_{0} \\
\gamma_{1} \\
\vdots \\
\gamma_{p-1}
\end{array}\right] \text {, }
$$

onde $f\left(x_{i}, \gamma\right)$ é a função não linear esperada, $x_{i}$ é o vetor de observações da variável independente (constantes conhecidas), $\gamma$ é o vetor de parâmetros do modelo $\gamma=\left(\gamma_{0}, \gamma_{1} \ldots, \gamma_{k}\right)^{T}$, e $\varepsilon_{i}$ é o termo do erro independente que se representa com uma distribuição normal de média nula e variância constante. Segundo Kutner et al. (2005) a importância dos modelos de regressão não-linear, é que o número de parâmetros não é necessariamente relacionado ao número de variáveis independentes $(x)$ do modelo estudado.

Para a estimação dos parâmetros é utilizado o método dos mínimos quadrados ou método de máxima verossimilhança, já que se requer dos critérios de minimização já mencionados anteriormente. Portanto, será utilizado o método dos mínimos quadrados que é representado por:

$Q=\sum_{i=1}^{n}\left[Y_{i}-f\left(x_{i}, \gamma\right)\right]^{2}$ 
onde $f\left(x_{i}, \gamma\right)$ é a média da variável resposta para $i$ da função de resposta não linear $f(x, \gamma)$. No critério dos mínimos quadrados, $Q$ deve ser minimizado em relação aos parâmetros da regressão linear $\gamma_{0}, \gamma_{1}, \ldots, \gamma_{p-1}$, para assim obter as estimativas do modelo.

\subsubsection{Heteroscedasticidade}

Quando é utilizado um modelo de regressão, seja linear ou não linear, o ideal é ter um conjunto de dados com comportamento homoscedastico (homo=igual, scedastico= dispersão). Neste comportamento a variância do erro é constante para todas as observações, conforme Equação (4.45). A homoscedasticidade é uma propriedade fundamental que é assumida na análise de regressão.

$$
E\left(u_{i}^{2}\right)=\sigma^{2} \quad i=1,2, \ldots, n .
$$

A maioria dos testes paramétricos supõe homoscedasticidade na variância dos erros. Isto é feito para que os modelos de regressão possam ser aplicados a qualquer conjunto de dados. Para esclarecer a homoscedasticidade, na Figura 4.4a é possível observar sua existência, pois a variância dos erros é uniforme para o modelo de regressão utilizado.

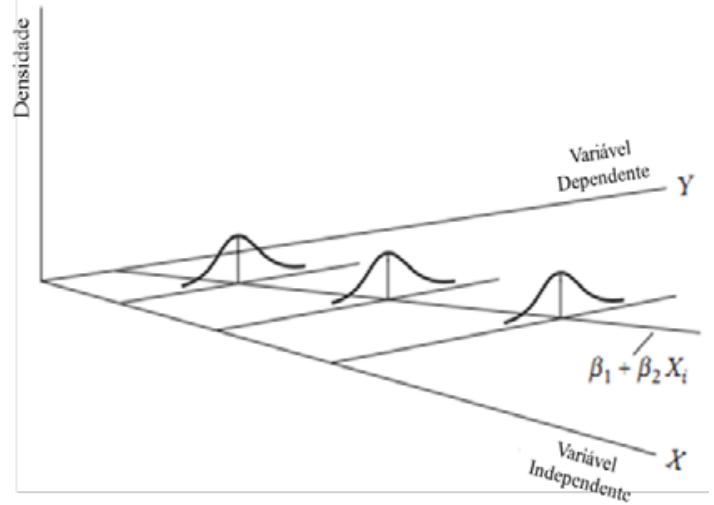

(a)

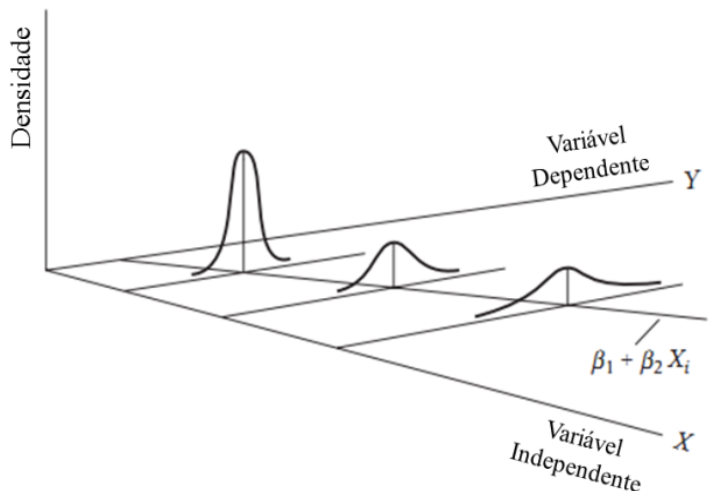

(b)

Figura 4.4. Distribuição (a) Homoscedastica. (b) Heteroscedastica.

[Gujarati e Porter, 2009 - adaptado]. 
Quando a variância do erro aumenta para cada observação se diz que existe heteroscedasticidade como representado na Figura 4.4b. A heteroscedasticidade é um problema estatístico que aparece com o aumento da variância na medida em que a variável independente aumenta (Equação (4.46)). A heteroscedasticidade apresenta-se como uma forte dispersão dos dados em torno ao modelo de ajuste, como observado na Figura 4.4b.

$$
E\left(u_{i}^{2}\right)=\sigma_{i}^{2}
$$

Para a detecção deste problema estatístico, há alguns métodos que ajudam a avaliar, se o conjunto de dados possui alguma tendência de aumento na variância. Alguns destes métodos para detectar tal presença são: o método gráfico, o teste de Glejser, o teste de coeficiente de correlação de Spearman, o teste de Goldfeld-Quandt, o teste de Breusch-Pagan-Godfrey, o teste geral de heteroscedasticidade de White, o teste de Koenker-Basset (KB) e o teste de Park. O método gráfico, o teste de White e o teste de KB são baseados no valor quadrático dos resíduos $\left(u_{i}{ }^{2}\right)$. Por meio destes resíduos, avalia-se se os dados apresentam um padrão sistemático. Quando nos dados se apresenta um padrão sistemático (Figura 4.5), afirma-se que há existência de heteroscedasticidade.
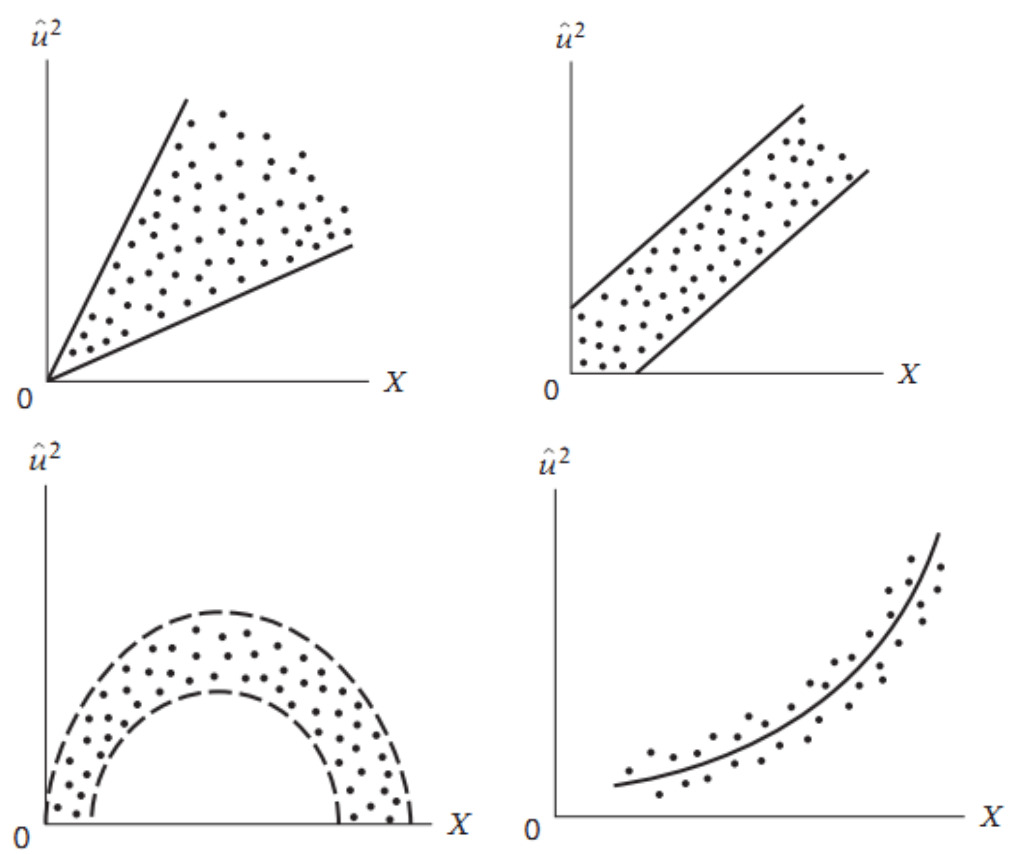

Figura 4.5. Padrões hipotéticos do valor quadrático dos resíduos estimados. [ Gujarati e Porter, 2009 - adaptado]. 
Para a solução deste problema estatístico, pode-se aplicar o método dos mínimos quadrados ponderados (MQP). Ressalta-se que o método dos mínimos quadrados ponderados é um método direto, que se utiliza para fazer correção da variância. Um modelo probabilístico linear múltipla utilizado é representado como:

$Y_{i}=\beta_{0}+\beta_{1} x_{i 1}+\cdots+\beta_{p-1} X_{i, p-1}+\varepsilon_{i}$,

onde $\beta_{0}, \beta_{1}, \ldots, \beta_{p-1}$ são os parâmetros, $x_{i 1}, x_{i 2}, \ldots, x_{i, p-1}$ são as constantes conhecidas, e $\varepsilon_{i}$ é a variável erro independente que apresenta uma distribuição $N\left(0, \sigma_{i}^{2}\right)$. Os estimadores do método dos mínimos quadrados podem ser obtidos pela redução dada por:

$\sum \hat{\varepsilon}_{i}^{2}=\sum\left(Y_{i}-\hat{\beta}_{0}-\hat{\beta}_{1} x_{i}\right)^{2}$

podendo ser escrita da seguinte forma:

$\sum\left(\frac{\hat{\varepsilon}_{i}}{\sigma_{i}}\right)^{2}=\sum\left[\left(\frac{Y_{i}}{\sigma_{i}}\right)-\hat{\beta}_{0}\left(\frac{1}{\sigma_{i}}\right)-\hat{\beta}_{1}\left(\frac{x_{i}}{\sigma_{i}}\right)\right]^{2}$.

O método dos mínimos quadrados faz uma redução para obter os estimadores da função de ajuste. Em contrapartida, o método dos mínimos quadrados ponderados faz uma redução do valor quadrático da soma ponderada dos resíduos, sendo representada por:

$\sum w_{i} \hat{\varepsilon}_{i}^{2}=\sum w_{i}\left(Y_{i}-\hat{\beta}_{0}^{*}-\hat{\beta}_{1}^{*} x_{i}\right)^{2}$,

onde $\hat{\beta}_{0}^{*}, \hat{\beta}_{1}^{*}$ são os estimadores obtidos a partir do método dos mínimos quadrados ponderados e $w_{i}$ são as ponderações que são inversamente proporcionais à variância e pode ser obtida como:

$w_{i}=\frac{1}{\sigma_{i}^{2}}$. 
Quando há presença de heteroscedasticidade, as variâncias são diferentes para cada observação. Isto pode ser representado por:

$\operatorname{Var}\left[\varepsilon_{i} \mid X_{i}\right]=\operatorname{Var}\left[Y_{i} \mid X_{i}\right]=\sigma_{i}^{2}$

Ao derivar a Equação (4.50) em relação aos $\hat{\beta}_{0}^{*}, \hat{\beta}_{1}^{*}$ obtém-se:

$\frac{\partial \sum w_{i} \hat{\varepsilon}_{i}^{2}}{\partial \hat{\beta}_{0}^{*}}=2 \sum w_{i}\left(Y_{i}-\hat{\beta}_{0}^{*}-\hat{\beta}_{1}^{*} x_{i}\right)(-1) ;$
$\frac{\partial \sum w_{i} \hat{\varepsilon}_{i}^{2}}{\partial \hat{\beta}_{1}^{*}}=2 \sum w_{i}\left(Y_{i}-\hat{\beta}_{0}^{*}-\hat{\beta}_{1}^{*} x_{i}\right)\left(-x_{i}\right)$.

Igualando a zero as Equações (4.53) e (4.54) pode-se obter as equações normais:

$$
\begin{aligned}
& \sum w_{i} Y_{i}=\hat{\beta}_{0}^{*} \sum w_{i}+\hat{\beta}_{1}^{*} \sum w_{i} x_{i} ; \\
& \sum w_{i} x_{i} Y_{i}=\hat{\beta}_{0}^{*} \sum w_{i} x_{i}+\hat{\beta}_{1}^{*} \sum w_{i} x_{i}^{2} .
\end{aligned}
$$

Finalmente, para obter os estimadores $\hat{\beta}_{0}{ }^{*}, \hat{\beta}_{1}{ }^{*}$ devem ser resolvidas as equações simultaneamente, para assim obter:

$$
\begin{aligned}
& \hat{\beta}_{1}^{*}=\frac{\left(\sum w_{i}\right)\left(\sum w_{i} x_{i} Y_{i}\right)-\sum w_{i} x_{i} \sum w_{i} Y_{i}}{\left(\sum w_{i}\right)\left(\sum w_{i} x_{i}^{2}\right)-\left(\sum w_{i} x_{i}\right)^{2}} ; \\
& \hat{\beta}_{0}^{*}=\frac{\sum w_{i} Y_{i}}{\sum w_{i}}-\frac{\sum w_{i} x_{i}}{\sum w_{i}}\left[\frac{\left(\sum w_{i}\right)\left(\sum w_{i} x_{i} Y_{i}\right)-\sum w_{i} x_{i} \sum w_{i} Y_{i}}{\left(\sum w_{i}\right)\left(\sum w_{i} x_{i}^{2}\right)-\left(\sum w_{i} x_{i}\right)^{2}}\right] .
\end{aligned}
$$

Estes estimadores são minimizados, e também garantem a presença de homoscedasticidade no conjunto de dados estudados. 


\subsection{Estados limites}

Segundo Beck (2012), uma estrutura deve cumprir uma determinada função estrutural durante sua vida útil, com um nível adequado de segurança e de maneira economicamente viável. Os requisitos básicos são: serviço, segurança, robustez, econômico e social.

Um modo de falha descreve uma maneira como um componente pode falhar, levando a estrutura a um estado indesejável, dando origem aos estados limites. Os estados limites podem ser de serviço ou último. Os estados limites de serviço correspondem às condições normais de uso que levam a danos, deformações, vibrações excessivas e outras condições que possam levar ao desconforto do usuário. O estado limite último corresponde a requisitos de segurança, que podem causar o uso da capacidade máxima, deformação da estrutura que podem levar o colapso ou não, tais características podem levar a estrutura a ficar em total insegurança.

Estes requisitos são importantes numa estrutura e podem ser quantificados através das equações de estado limite. Estas equações de estado limite $g()$ devem ser estabelecidas para cada modo de falha, $\boldsymbol{g}_{i}(\boldsymbol{X})$, que é função das variáveis do projeto $\boldsymbol{X}=\left[X_{1}, X_{2}, \ldots, X_{n}\right]$. A equação de estado limite é dada por:

$g(X)=g\left(X_{1}, X_{2}, \ldots, X_{n}\right)$.

A equação de estado limite é escrita de tal forma, que exista uma fronteira entre os domínios de falha e segurança. Os domínios são dados por: 


$$
\begin{aligned}
& D_{f}=\{x \mid g(x) \leq 0\} ; \\
& D_{s}=\{x \mid g(x)>0\} .
\end{aligned}
$$

Na Figura 5.1 apresenta-se a fronteira entre os domínios de falha e segurança no espaço amostral das variáveis aleatórias.

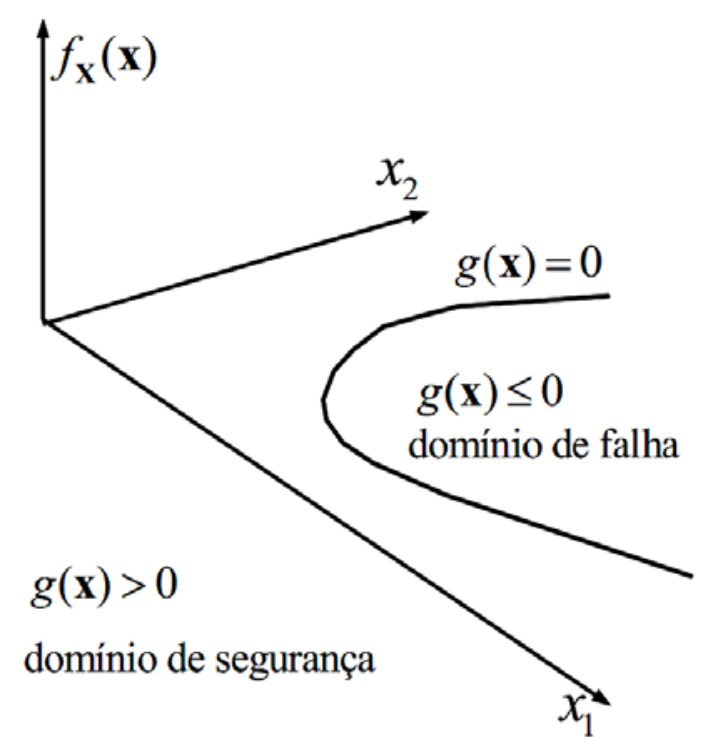

Figura 5.1. Equação de estado limite e domínios de falha e segurança [Beck, 2012].

A formulação apresentada nas Equações (5.2) e (5.3) se aplica para problemas independentes do tempo.

\subsection{Problema fundamental de confiabilidade estrutural independente do tempo}

O problema fundamental de confiabilidade também pode ser resolvido através da variável margem de segurança $(M)$. Relacionando duas variáveis aleatórias não correlacionadas às quais são a resistência $(R)$ e solicitação $(S)$. Tratando-se $M$ como uma variável aleatória sendo definida pela expressão a seguir:

$M=R-S$. 
Assim, a probabilidade de falha para tal problema é calculada a partir de $M$, e apresentada por:

$P_{f}=P[\{M \leq 0\}]=\int_{-\infty}^{0} f_{M}(m) d m=F_{M}(0)$.

Se $R$ e $S$ são variáveis aleatórias normais independentes, podem-se calcular os parâmetros da variável $M$, da seguinte forma:

$\mu_{M}=\mu_{R}-\mu_{S}$

$\sigma_{M}=\sqrt{\sigma_{R}^{2}+\sigma_{S}^{2}}$.

Por meio da transformação de Hasofer-Lind, a variável aleatória normal $M$ pode ser transformada em uma variável normal padrão $Y$, que possui média nula e desvio padrão unitário. A variável normal padrão $Y$ é obtida como:

$Y=\frac{M-\mu_{M}}{\sigma_{M}}$.

Através da Equação (5.8) é possível avaliar probabilidades associadas à variável normal padrão, por meio da função de distribuição acumulada normal padrão, $\Phi($ ). Logo, tem-se a expressão:

$P f=P[\{M \leq 0\}]=P\left(Y \leq-\frac{\mu_{M}}{\sigma_{M}}\right)=\Phi\left(-\frac{\mu_{M}}{\sigma_{M}}\right)=\Phi(-\beta)$.

Assim, pode-se obter:

$\beta \equiv \frac{\mu_{M}}{\sigma_{M}}=\frac{\mu_{R}-\mu_{S}}{\sqrt{\sigma_{R}^{2}+\sigma_{S}^{2}}}$. 
Observa-se na Equação (5.10) que a razão $\frac{\mu_{M}}{\sigma_{M}}$ é a distância entre o ponto $m=0$ e a origem da distribuição $Y$, esta medida é chamada como índice de confiabilidade, representada por $\beta$ (Beck, 2012).

O índice de confiabilidade $(\beta)$ representa uma medida geométrica da probabilidade de falha, que minimiza a distância entre o ponto de projeto e a origem do espaço normal padrão $Y$ conforme Figura 5.2.

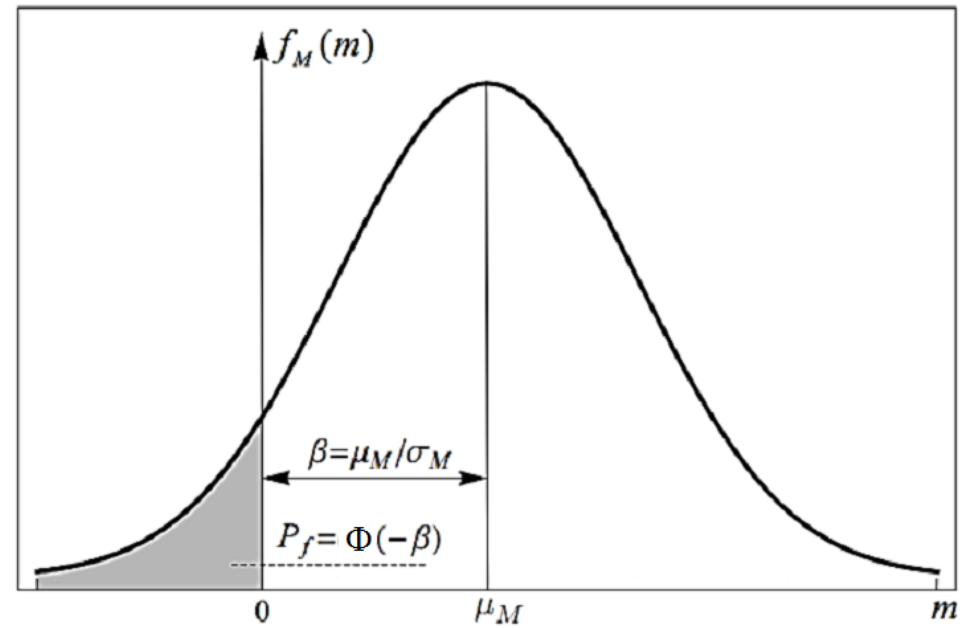

Figura 5.2. Probabilidade de em termos da variável normal padrão $Y$. [Beck, 2012 adaptado]

O ponto de projeto (design point ou Most Probable (failure) Point (MPP)) pode ser definido como o ponto sobre a superfície de falha que denota a menor distância à origem do espaço normal padrão. No espaço normal padrão a função de distribuição de probabilidades conjunta atinge simetria radial. O ponto de projeto é o ponto sobre o domínio de falha com maior probabilidade de ocorrência, ou seja, é o ponto que guarda o maior conteúdo de probabilidade.

\subsection{Aproximação de Primeira Ordem e Segundo Momento (FOSM)}

Na análise pela aproximação de Primeira Ordem e Segundo Momento (FOSM) a equação de estado limite é linearizada no ponto de projeto por uma função linear. Neste ponto a transformação das variáveis por Hasofer-Lind é utilizada (Equação (5.8)). Assim, realiza-se a 
transformação das variáveis aleatórias $R$ e $S$ nas variáveis no espaço normal padrão $Y_{1}$ e $Y_{2}$ através da Equação (5.8). A expressão margem de segurança é apresentada pela Figura 5.3 e dada por:

$$
m(r, s)=r-s=g\left(y_{1}, y_{2}\right)=y_{1} \sigma_{R}+\mu_{R}-y_{2} \sigma_{S}-\mu_{S} .
$$
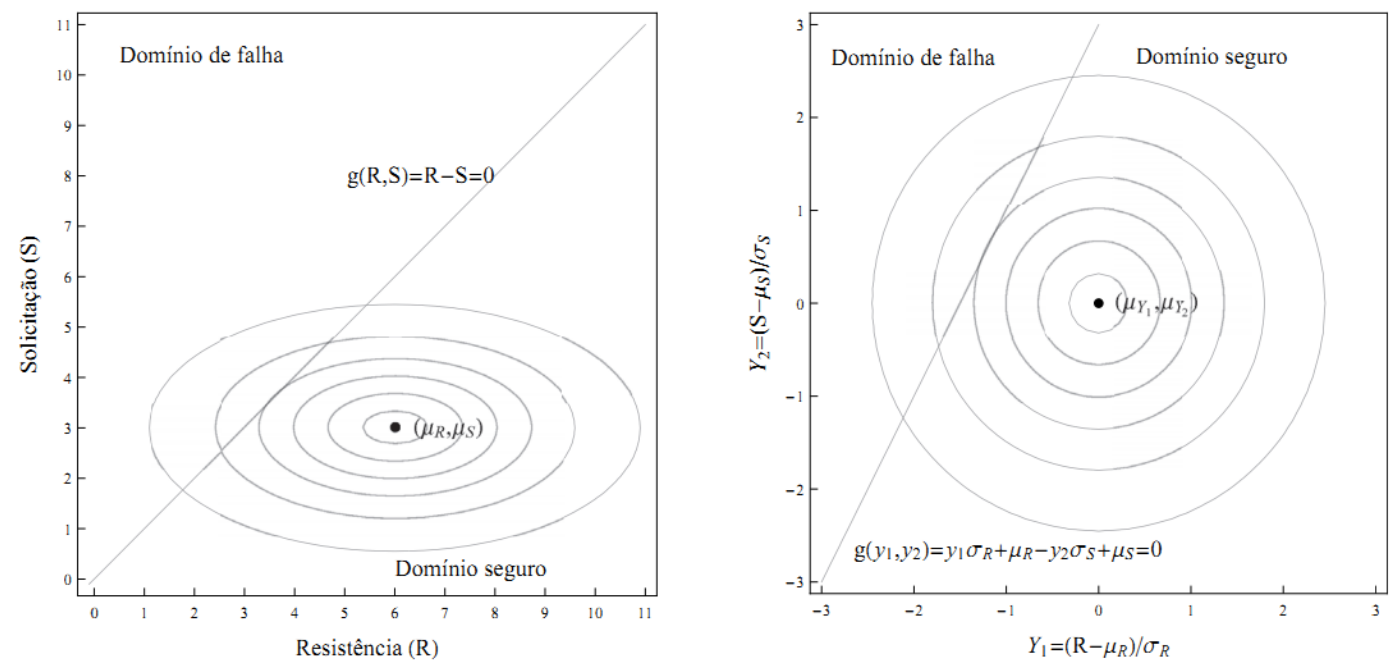

Figura 5.3. Transformação das variáveis $\mathrm{R}$ e $\mathrm{S}$ em variáveis normais padrão $Y_{1}, Y_{2}$ [Beck, 2012].

Fazendo $m\left(y_{1}, y_{2}\right)=0$ sendo uma linha reta apresentada na Figura 5.3, é dada por:

$y_{1} \sigma_{R}+\mu_{R}-y_{2} \sigma_{S}-\mu_{S}=0$.

O quadrado da distância entre um ponto qualquer $\left(y_{1}, y_{2}\right)$ e a origem é dado por $d^{2}=y_{1}^{2}+y_{2}^{2}$. Realizando a derivada em relação a $y_{1}$ e igualando a zero, obtemos as coordenadas $y_{1}^{*}$ do ponto sobre a equação $m\left(y_{1}, y_{2}\right)=0$ mais próxima da origem, sendo dada por:

$$
\begin{aligned}
& y_{1}^{*}=-y_{2} \frac{\sigma_{R}}{\sigma_{S}} ; \\
& y_{1}^{*}=-\frac{\sigma_{R}\left(\mu_{R}-\mu_{S}\right)}{\sigma_{R}^{2}+\sigma_{S}^{2}} .
\end{aligned}
$$


Derivando em relação a $y_{2}$ e igualando a zero, obtemos as coordenadas $y_{2}{ }^{*}$ do ponto sobre a equação $m\left(y_{1}, y_{2}\right)=0$ mais próxima da origem, como:

$y_{2}^{*}=-y_{1} \frac{\sigma_{S}}{\sigma_{R}}$

$y_{2}^{*}=-\frac{\sigma_{S}\left(\mu_{R}-\mu_{S}\right)}{\sigma_{R}^{2}+\sigma_{S}^{2}}$.

Substituindo as Equações (5.14) e (5.16) na expressão $d^{2}=y_{1}{ }^{2}+y_{2}{ }^{2}$ e tomando a raiz, obtém-se a mínima distância da superfície de falha à origem, da seguinte forma:

$d_{\min }=\frac{\mu_{R}-\mu_{S}}{\sqrt{\sigma_{R}^{2}+\sigma_{S}^{2}}}$.

Nota-se que a Equação (5.17) é igual à Equação (5.10). As duas indicam o termo índice de confiabilidade.

\subsection{Algoritmo de Hasofer, Lind, Rackwitz e Fiessler}

A fórmula recursiva está baseada na aproximação de um ponto qualquer y à superfície $g(y)=0$ e na perpendicularização entre o valor $y$ e a tangente à superfície no ponto (Beck, 2012). Sendo $y_{k}$ um ponto inicial qualquer que se encontra por fora da superfície de falha. A expansão da equação de estado limite se aproxima com os termos de primeira ordem na série de Taylor, conforme equação a seguir:

$\tilde{g}\left(y_{k+1}\right) \approx g\left(y_{k}\right)+\nabla g\left(y_{k}\right)^{T} \cdot\left(y_{k+1}-y_{k}\right)$,

sendo $\nabla g\left(y_{k}\right)$ o gradiente da equação de estado limite (no espaço normal padrão), avaliado no ponto $y_{k}$. Para a equação linearizada deve-se encontrar um novo ponto $y_{k+1}$, de tal forma que $\tilde{g}\left(y_{k+1}\right)=0$. O valor inicial do índice de confiabilidade é obtido como $\beta_{k}=\sqrt{y_{k}^{T} \cdot y_{k}}$ e o 
vetor de cossenos diretores da equação de estado limite $\left(\alpha_{k}\right)$ avaliado no ponto $y_{k}$ é dado por:

$\alpha_{k}=\frac{\nabla g\left(y_{k}\right)}{\left\|\nabla g\left(y_{k}\right)\right\|}$.

Portanto, o novo ponto pode ser encontrado através da expressão a seguir:

$$
y_{k+1}=-\alpha_{k}\left[\beta_{k}+\frac{g\left(y_{k}\right)}{\left\|\nabla g\left(y_{k}\right)\right\|}\right] \text {. }
$$

O termo entre colchetes representa a nova aproximação do índice de confiabilidade $\left(\beta_{k+1}\right)$. A Equação (5.20) é utilizada de forma iterativa ate atingir a convergência em $y$ ou em $\beta$. Embora, o algoritmo HLRF seja o mais utilizado para encontrar o ponto de projeto, ele não garante a convergência, nem garante que seja encontrado o ponto de projeto. Portanto, devem ser feitas algumas modificações no algoritmo original.

\subsubsection{Aproximação de Primeira Ordem (FORM)}

O FORM é um método utilizado em confiabilidade para calcular a probabilidade de falha por meio da transformação das variáveis aleatórias.

O FORM utiliza a maior parte dos resultados apresentados para o FOSM. No método FORM (First Order Reliability Method) a superfície de falha é aproximada por um hiperplano no ponto de projeto. É fundamental a utilização de toda informação estatística das variáveis envolvidas no problema, o que inclui variáveis com distribuições marginais não normais (não gaussianas) e coeficientes de correlação entre pares de variáveis.

O FORM se baseia na construção de uma função conjunta de distribuição de probabilidades $f_{X}(x)$ e a sua transformação para o espaço normal padrão $Y$. A transformação envolve a eliminação da correlação entre variáveis aleatórias e o cálculo das variáveis normais equivalentes. E ela representa um mapeamento um a um, levando os pontos do espaço de projeto $\mathbb{X}$ para o espaço normal padrão $\mathbb{Y}$. Para se construir a função conjunta de distribuição de probabilidades no espaço de projeto $\mathbb{X}$, geralmente são conhecidas duas propriedades, que 
são as distribuições de probabilidades marginais $f_{X_{i}}\left(x_{i}\right)$ de cada variável aleatória e a matriz de correlação $R_{X}$ entre pares de variáveis, dada por:

$$
R_{X}=\left[\begin{array}{cccc}
1 & \rho_{X_{12}} & \cdots & \rho_{X_{1 n}} \\
\rho_{X_{21}} & 1 & \cdots & \rho_{X_{2 n}} \\
\vdots & \vdots & \ddots & \vdots \\
\rho_{X_{n 1}} & \rho_{X_{n 2 i}} & \cdots & 1
\end{array}\right]
$$

sendo $n$ o número de variáveis aleatórias envolvidas no problema (Beck, 2012).

\subsubsection{Transformação composta utilizando o modelo de Nataf}

Este modelo é utilizado quando as variáveis aleatórias possuem qualquer tipo de distribuição. O princípio de aproximar a cauda da distribuição original pela cauda de uma distribuição normal equivalente é conhecido na literatura como o princípio de aproximação normal Principle of normal tail approximation (Ditlevsen, 1981). O princípio consiste em determinar, no ponto $x_{i}^{*}$, uma distribuição normal equivalente que preserva o conteúdo de probabilidades da distribuição original $F_{X_{i}}\left(x_{i}^{*}\right)$. Os parâmetros da distribuição normal equivalente são dados por:

$$
\begin{aligned}
& \sigma_{X_{i}}^{n e q}=\frac{\phi\left(z_{i}{ }^{*}\right)}{f_{X_{i}}\left(x_{i}{ }^{*}\right)}, \\
& \mu_{X i}^{n e q}=x_{i}{ }^{*}-z_{i}{ }^{*} \sigma_{X_{i}}^{\text {neq }},
\end{aligned}
$$

onde $\phi$ é a função de probabilidades normal padrão, $z^{*}$ é o ponto correspondente a $x^{*}$ no espaço normal padrão, $\sigma_{X_{i}}^{\text {neq }}$ é o desvio padrão da distribuição normal equivalente e $\mu_{X_{i}}^{\text {neq é a }}$ média da distribuição normal equivalente. Para transformar um ponto de $\mathbb{X} \rightarrow \mathbb{Z}$ ou o inverso utiliza-se:

$$
z_{i}^{*}=\Phi^{-1}\left[F_{X_{i}}\left(x_{i}^{*}\right)\right]
$$


Esta transformação deve ser realizada para cada uma das distribuições marginais, sendo válida para um ponto $x^{*}$, de tal forma que à medida que o algoritmo de busca do ponto de projeto avança o ponto $x^{*}$ muda. Esta transformação de $\mathbb{X} \rightarrow \mathbb{Z}$ pode ser definida matricialmente a partir do vetor de médias $M_{\text {neq }}$ e de uma matriz diagonal de desvios padrões $D_{\text {neq }}$, dado por:

$$
\begin{aligned}
M^{\text {neq }} & =\left\{\begin{array}{cccc}
\mu_{X_{1}}^{\text {neq }} & \mu_{X_{2}}^{\text {neq }} & \cdots & \mu_{X_{n}}^{\text {neq }}
\end{array}\right\}^{T} ; \\
D^{\text {neq }} & =\left[\begin{array}{cccc}
\sigma_{X_{1}}^{\text {neq }} & 0 & \cdots & 0 \\
0 & \sigma_{X_{2}}^{\text {neq }} & \cdots & 0 \\
\vdots & \vdots & \ddots & \vdots \\
0 & 0 & \cdots & \sigma_{X_{n}}^{\text {neq }}
\end{array}\right] .
\end{aligned}
$$

A Partir da Equação (5.26) obtêm-se as matrizes jacobianas, dada por:

$$
\begin{aligned}
& J_{Z X}=\left(D^{\text {neq }}\right)^{-1} ; \\
& J_{X Z}=D^{\text {neq }} .
\end{aligned}
$$

As transformações $\mathbb{X} \rightarrow \mathbb{Z}$ e $\mathbb{Z} \rightarrow \mathbb{X}$, são obtidas cômoda maneira a seguir:

$$
\begin{aligned}
& Z=J_{Z X} \cdot\left\{X-M^{\text {neq }}\right\} ; \\
& X=J_{X Z} \cdot Z+M^{\text {neq }} .
\end{aligned}
$$

A partir do modelo de Nataf, permite-se construir uma aproximação para a função conjunta de densidade de probabilidades $f_{X}(x)$ com a distribuição normal padrão multivariada e com a matriz de correlação $R_{z}$. A função de densidade de probabilidade $f_{X}(x)$, é dada por:

$$
f_{X}(x)=\phi_{n}\left(z, R_{z}\right) \frac{f_{X_{1}}\left(x_{1}\right) f_{X_{2}}\left(x_{2}\right) \ldots f_{X_{n}}\left(x_{n}\right)}{\phi\left(z_{1}\right) \phi\left(z_{2}\right) \ldots \phi\left(z_{n}\right)} .
$$

Os coeficientes de correlação $\rho_{i j}$ são obtidos utilizando a definição de covariância de maneira iterativa como é mostrada na expressão a seguir: 
$\rho_{X_{i j}}=\int_{-\infty}^{\infty} \int_{-\infty}^{\infty} z_{i} z_{j} \phi_{2}\left(z_{i}, z_{j}, \rho_{z_{i j}}\right) d z_{i} d z_{j}$

onde $\phi_{2}$ é a distribuição normal padrão bivariada. Portanto, a partir da Equação (5.32) podese obter $\rho_{Z_{i j}}$ em função do $\rho_{X_{i j}}$.

A última etapa consiste em eliminar a correlação entre os pares de variáveis aleatórias, passando-as do espaço $\mathbb{Z} \rightarrow \mathbb{Y}$. Este procedimento pode ser feito através de uma decomposição ortogonal ou através da decomposição de Cholesky da matriz de correlação. Tendo em vista o elevado custo computacional existente na decomposição ortogonal, será apresentada a fatoração de Cholesky que possui um custo computacional menor. Isto se deve ao número reduzido de operações matriciais e com vantagem para matrizes de correlação não cheias. Utilizando a decomposição de Cholesky de $C_{Z}$, tem-se que:

$C_{Z}=L \cdot L^{T}$

Assim, obtêm-se as matrizes jacobianas via decomposição Cholesky de $C_{Z}$ (Beck, 2012):

$$
\begin{aligned}
& J_{y z}=L^{-1} ; \\
& J_{z y}=L .
\end{aligned}
$$

Logo, a transformação resultante de $\mathbb{Y} \rightarrow \mathbb{Z}$ e $\mathbb{Z} \rightarrow \mathbb{Y}$ são:

$$
\begin{aligned}
& y=J_{y z} \cdot z, \\
& z=J_{z y} \cdot y .
\end{aligned}
$$




\subsubsection{Algoritmo FORM}

Para soluções de problemas de confiabilidade independentes do tempo via FORM, tem-se a realização das seguintes etapas:

- Determinação dos coeficientes de correlação equivalentes e da matriz de decomposição; determinação das matrizes jacobianas $J_{y z}$ e $J_{z y}$;

- Escolha o ponto inicial $x_{k}$ para $k=0$ (usualmente o ponto médio)

- Determinação dos parâmetros das distribuições normais equivalentes no ponto $x_{k}$ (matrizes $M^{\text {neq }}$ e $D^{\text {neq }}$ ).

- Atualização das matrizes jacobianas $J_{y x}$ e $J_{x y}$.

- Transformação do ponto $x_{k}$ de $\mathbb{X}$ para $\mathbb{Y}$.

- Avaliação de $g\left(x_{k}\right)$.

- Cálculo do gradiente $\left[\nabla g\left(y_{k}\right)\right]$

o Cálculo das derivadas parciais de $g(x)$ no espaço de projeto $\mathbb{X}$.

o Transformação do gradiente para o espaço normal padrão $\mathbb{Y}$.

o Cálculo dos fatores de sensibilidade $\alpha\left(y_{k}\right)$. Estes fatores podem ser utilizados para eliminar o problema de variáveis aleatórias que tenham pouca influência na probabilidade de falha.

- Cálculo de novo ponto $y_{k+1}$ pelos algoritmos HLRF;

- Transformação de $y_{k+1}$ para $\mathbb{X}$;

- Verificação do critério de convergência. Se:

$$
0 \quad 1+\left|\frac{\nabla g\left(y_{k+1}\right) \cdot y_{k+1}}{|| \nabla g\left(y_{k+1}\right)\|\cdot\| y_{k+1} \|}\right|<\in \quad \text { e } \quad\left|g\left(y_{k+1}\right)\right|<\delta,
$$

o algoritmo é interrompido, caso contrário retorna-se à determinação dos parâmetros com $k=k+1$ até atingir a convergência.

- Ao final, é feita a avaliação do índice de confiabilidade no ponto de projeto: $\beta=\left\|y^{*}\right\|$. 


\subsection{Análise das amostras}

Em todo estudo de dados o mais desejável é contar com uma grande base de dados, já que o erro estatístico pode ser minimizado à medida que se aumenta o tamanho da amostra. No presente trabalho a base de dados, apresentada no capítulo 3, consta de 427 dados obtidos de ensaios em dutos que contêm defeito de corrosão real e artificial. O tamanho da amostra é importante para obter uma dependência das variáveis fundamentais nos modelos estudados.

A seguir será apresentado um estudo preliminar para se determinar, se os defeitos reais e artificiais podem ser tratados em conjunto. Para isso, o teste de Kolmogorov-Smirnov (K-S) será utilizado e, portanto, ele determinará se as amostras pertencem a mesma população.

Na Figura 6.1 pode ser visto a função de distribuição acumulada da variável erro do modelo para os defeitos reais e artificiais.

A partir da Figura 6.1 e da Equação (4.30) pode ser obtido o parâmetro estatístico do teste KS. A Tabela 6.1, explicita os parâmetros do teste K-S calculados para os modelos semiempíricos que estão sendo avaliados. Estes parâmetros devem ser comparados com os valores $D_{\text {Critico }}$ que dependem de níveis de significância iguais a 1\%, 5\%, 10\%, 15\% e/ou 20\%, encontrados na tabela do ANEXO A. Segundo o critério apresentado da hipótese nula, tem-se que ela é aceita quando: $D_{\text {Max }} \leq D_{\text {Critico }}$ ou o valor-p é maior que o nível de significância que está sendo comparado. No entanto, a relação $D_{\text {Max }} \leq D_{\text {Critico }}$ deve ser a primeira analisada, e para o caso que ela é aceita não é necessário verificar o valor-p. 


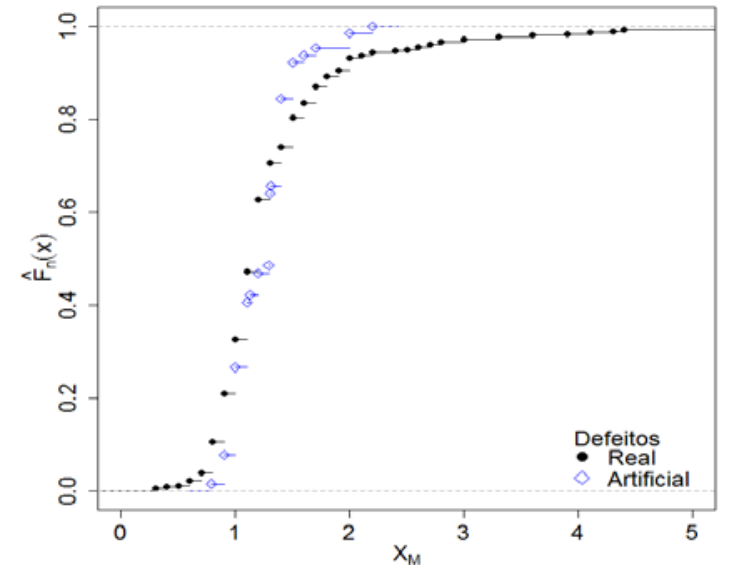

(a)

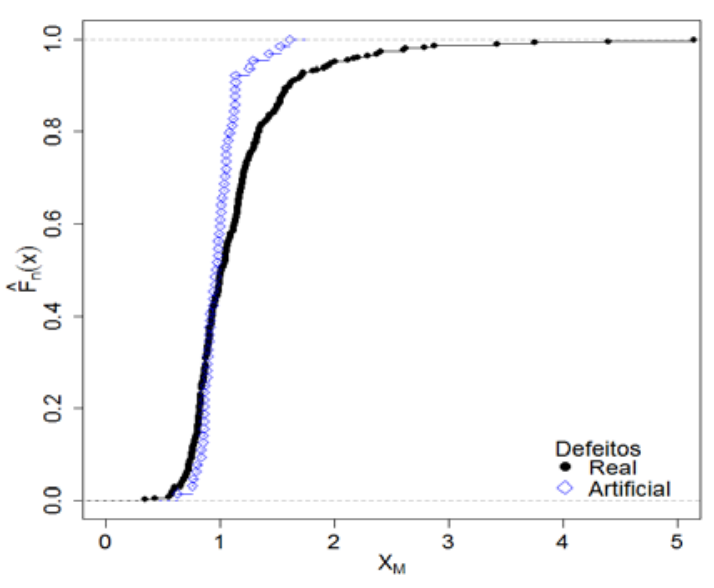

(c)

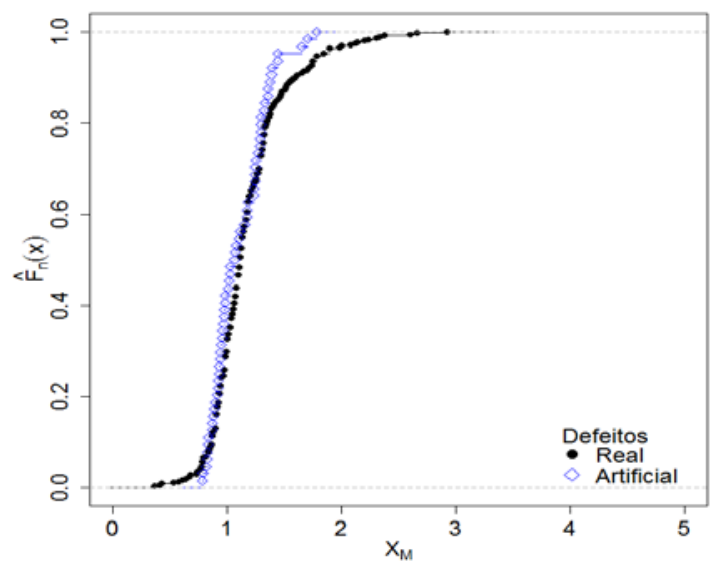

(b)

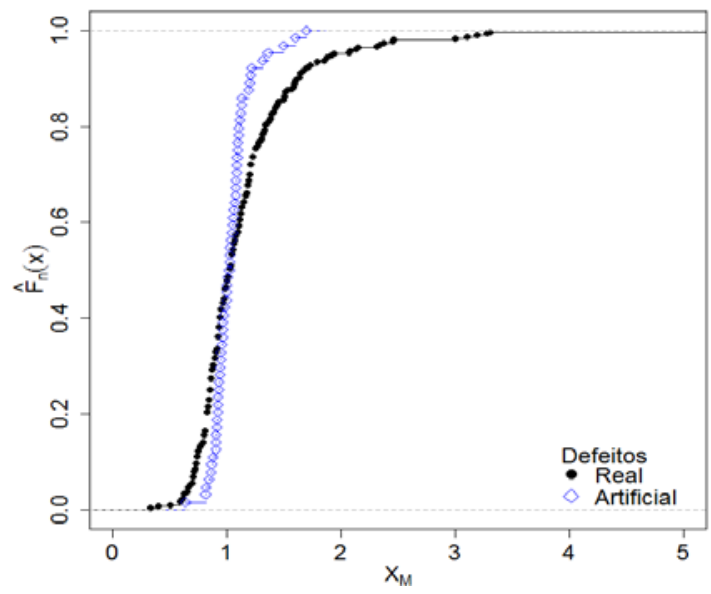

(d)

Figura 6.1. Função de distribuição acumulada de probabilidades pelos modelos semiempíricos (a) ASME B31G. (b) B31G modificado. (c) DNV RP F101. (d) PCORRC.

Tabela 6.1. Parâmetros estatísticos do teste Kolmogorov-Smirnov.

\begin{tabular}{lll}
\hline Modelo & D $_{\text {Max }}$ & Valor-p \\
\hline ASME B31G & 0,1583 & 0,1309 \\
ASME B31G modificado & 0,1346 & 0,2782 \\
DNV RP F101 & 0,3045 & 0,0001062 \\
PCORRC & 0,241 & 0,004314 \\
\hline
\end{tabular}

Seguindo o raciocínio, o modelo ASME B31G apresenta um $D_{\text {Max }}(0,1583)$ maior que o $D_{\text {Crit }}$ $(0,05184)$ para o nível de significância de $20 \%$. Entretanto, o valor-p $(0,1309)$ é maior que o nível de significância (10\%), o que confirma que estas amostras são da mesma população.

O mesmo acontece para o modelo B31G modificado, que apresenta um $D_{\operatorname{Max}}(0,1346)$ maior que o $D_{\text {Crit }}(0,05184)$ para o nível de significância de $20 \%$, e valor-p $(0,2782)$ maior que o 
nível de significância de 20\%. Fato que leva concluir que para este modelo as amostras também pertencem à mesma população.

Para os modelos DNV-RP-F101 e PCORRC o parâmetro estatístico $D_{\text {Max }}(0,3045)$ e $(0,241)$ é maior do que o $D_{\text {Critico }}(0,055)$ para o nível de significância $20 \%$. E o valor-p $(0.0001062)$ e $(0,004314)$, são menores que o nível de significância de $1 \%$. Isto confirma que as amostras não satisfazem a hipótese nula, e que elas não pertencem à mesma população.

Comprova-se, portanto, que as amostras com defeitos reais e defeitos artificiais, para os modelos ASME B31G e B31G modificado, podem ser tratadas em conjunto, tendo em vista que elas representam uma mesma população. Em contrapartida, os modelos DNV RP F101 e PCORRC devem ser analisados separadamente, e, portanto, apenas os defeitos reais serão aqueles utilizados para as análises.

O estudo de robustez apresentado (teste K-S) é importante porque gera uma maior confiança nas análises posteriores. Por meio dele determinou-se a quantidade de dados que será utilizada neste trabalho, que se encontra apresentada na Tabela 6.2.

Tabela 6.2. Quantidade de dados utilizada para as análises.

\begin{tabular}{llll}
\hline Modelo & Real & Artificial & Total \\
\hline ASME B31G & 363 & 64 & 427 \\
ASME B31G Modificado & & & \\
\hline DNV RP F101 & 311 & --- & 311 \\
PCORRC & & & \\
\hline
\end{tabular}

A seguir será apresentado um estudo que determina se existe ruptura ou vazamento no duto. Este estudo é feito a partir da razão $d / t$, que quando apresenta um valor próximo da unidade, existe ruptura ou vazamento. É apropriado utilizar os dados numa faixa que possa ser útil para as análises e estudos no futuro. Neste trabalho será tomado o critério da recomendação prática DNV RP F101, o qual prediz que a profundidade do defeito não pode exceder o 85\% da espessura da parede. Deste modo, os dados considerados são apenas aqueles que atendem à 
condição $d / t \leq 0,85$. Após esta filtragem tem-se uma menor quantidade de dados, como pode ser observado na Tabela 6.3.

Tabela 6.3. Quantidade de dados para a razão $d / t \leq 0.85$.

\begin{tabular}{lc}
\hline Modelo & Total \\
\hline ASME B31G & 405 \\
ASME B31G modificado & \\
\hline DNV RP F101 & 295 \\
PCORRC & 289 \\
\hline
\end{tabular}

Os modelos ASME B31G e B31G modificado possuem a mesma quantidade de dados, 405 cada. Os modelos DNV RP F101 e PCORRC apesar de conter parâmetros similares apresentam quantidades de dados diferentes, sendo 295 e 289, respectivamente.

\subsection{Caracterização da variável erro de modelo}

Após a avaliação estatística da variável erro de modelo por meio da Equação (2.1), realizou-se a caracterização desta variável aleatória. O ajuste de distribuição estatística foi realizado através do programa EasyFit 5.5, onde foram construídos histogramas e através destes foram realizados os testes de aderência, apresentados na seção 4.2. Para realizar os ajustes, se escolhe a distribuição que melhor se adequa ao comportamento da variável aleatória. $\mathrm{Na}$ Figura 6.2, são apresentados os histogramas que descrevem o comportamento da variável erro de modelo, junto com a função de densidade de probabilidades (FDP).

Na Tabela 6.4, encontram-se as distribuições de probabilidades e seus respetivos parâmetros que melhor se adequam a cada modelo. O valor médio, o desvio padrão e o COV são parâmetros estatísticos calculados para todos os modelos considerados. Estes resultados foram obtidos para as quantidades de dados apresentadas na Tabela 6.3. 


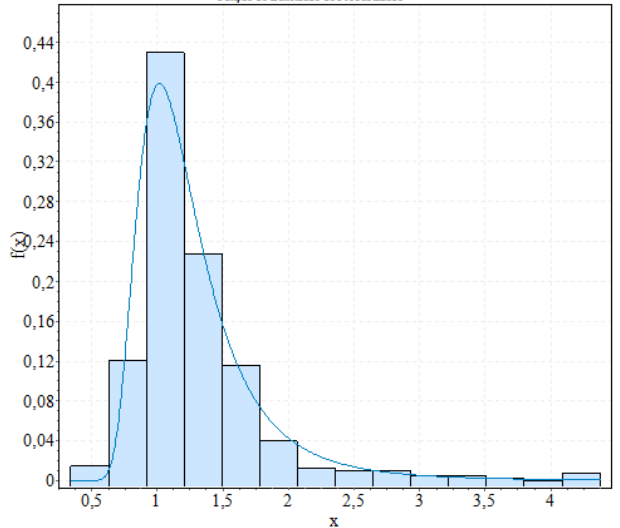

(a)

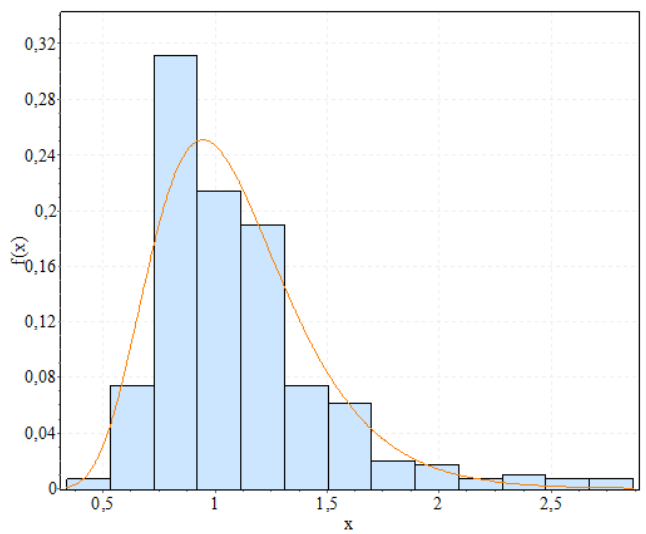

(c)

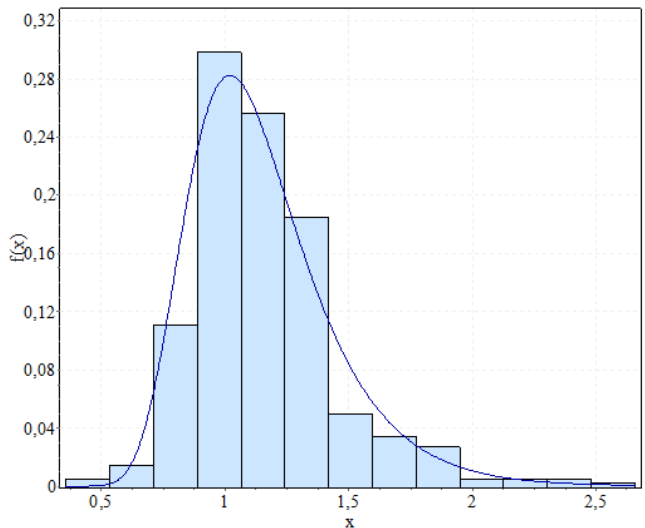

(b)

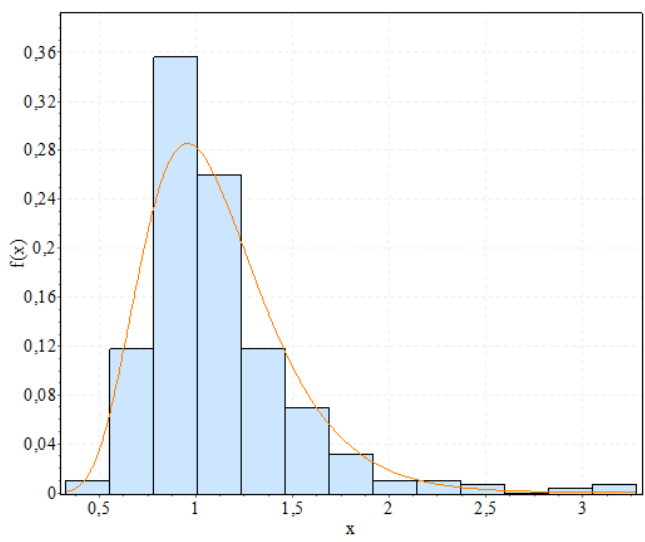

(d)

Figura 6.2. Histograma da variável erro de modelo e distribuição de probabilidades para os modelos (a) ASME B31G. (b) ASME B31G modificado. (c) DNV RP F101. (d) PCORRC.

Tabela 6.4. Caracterização da Variável Erro de Modelo $(d / t \leq 0,85)$.

\begin{tabular}{ccccc}
\hline MODELO & DISTRIBUIÇÃO & $\mu$ & COV & $\sigma$ \\
\hline B31G & Gen Extreme Value & 1,2889 & 0,3878 & 0,499 \\
B31G Mod & Gumbel & 1,152 & 0,256 & 0,295 \\
DNV & Lognormal & 1,0937 & 0,319 & 0,349 \\
PCORRC & Lognormal & 1,1089 & 0,324 & 0,359 \\
\hline
\end{tabular}

Analisando os resultados da Tabela 6.4, o modelo ASME B31G apresenta resultados excessivamente conservadores $(1,2889)$, tendo em vista que seu valor médio é bem maior que a unidade. Além disto, este modelo apresenta menor precisão na previsão estatística, pois o COV (em torno de 38\%) evidencia uma grande dispersão. Por outro lado, o modelo ASME B31G modificado é mais preciso que o anterior, pois a variável erro de modelo apresenta um 
valor médio (1,152), ou seja, menos conservador e COV (25,6\%) com menor dispersão que o modelo B31G.

Os modelos DNV e PCORRC apresentam valores altos de COV (31,9\% e 32,4\%) em comparação ao B31G modificado (25,5\%). Em contrapartida, o DNV e PCORRC apresentam um valor médio (1,0937 e 1,1089) menos conservador que os modelos B31G e B31G modificado (1,2889 e 1,152).

De maneira geral, comparando os quatro modelos, tem-se que o DNV é menos conservador que os B31G, B31G modificado e PCORRC. Ele apresenta um valor médio $(1,0937)$ um pouco maior que a unidade. Este modelo adota uma equação menos conservadora para obter o fator de dilatação e utiliza a resistência à tração última $\left(\sigma_{u}\right)$, enquanto os métodos B31G e B31G modificado utilizam a resistência de escoamento $\left(\sigma_{y}\right)$.

Os ajustes por meio dos testes estatísticos foram realizados para cada modelo (ASME B31G, B31G modificado, DNV, PCORRC), de modo a obter uma representação dos dados da variável aleatória erro de modelo para a quantidade de dados apresentada na Tabela 6.3.

\subsection{Comparação com resultados da literatura}

Os resultados obtidos por Zhou e Huang (2012), Tabela 6.5, mostram que, dentre os modelos apresentados, o B31G é o mais conservador associado a uma baixa precisão (média $(1,473)$ e COV (41,2\%)). O B31G modificado possui a mesma quantidade de dados do B31G, mas apresenta maior precisão, já que o COV (25,8\%) é menor. Ele também é menos conservador no valor médio dos dados $(1,297)$.

Com relação ao modelo DNV pode ser visto que ele apresenta um COV igual a 37,4\% e uma média igual a 1,233. Estes valores são relativamente maiores que todos os outros modelos analisados, exceto o B31G.

Ao analisar o modelo PCORRC os autores concluíram que ele é o menos conservador dentre os modelos de ruptura que não requerem o perfil do defeito. Ele apresenta o valor médio, próximo à unidade, igual a 1,079. 
Tabela 6.5. Estatística básica da variável erro de modelo (Zhou; Huang, 2012).

\begin{tabular}{lllll}
\hline Modelo & $\begin{array}{l}\text { Quantidade de dados } \\
\text { utilizados }\end{array}$ & Media & COV (\%) & Ajuste de distribuição \\
\hline B31G & 149 & 1,473 & 41,2 & Frechet \\
B31G Modificado & 149 & 1,297 & 25,8 & Gumbel \\
CPS & 41 & 1,228 & 19,8 & Frechet \\
Modelo CSA & 61 & 1,103 & 17,2 & Lognormal \\
DNV & 106 & 1,233 & 37,4 & Lognormal \\
PCORRC & 68 & 1,079 & 26,4 & Gumbel \\
RSTRENG & 98 & 1,067 & 16,5 & Normal \\
SHELL92 & 105 & 1,544 & 35,8 & Lognormal \\
\hline
\end{tabular}

Comparando os resultados apresentados na Tabela 6.4 e Tabela 6.5 é possível observar que eles são similares. Contudo, neste trabalho o modelo DNV é menos conservador, diferentemente do concluído no trabalho de Zhou e Huang (2012) que afirmam que seria o modelo PCORRC. Ressalta-se que o modelo é menos conservador quando o seu valor médio encontra-se acima, mas mais próximo da unidade.

A diferença entre os resultados pode ser explicada porque Zhou e Huang (2012) utilizam para o modelo PCORRC uma base de dados menor (68 dados) que para o DNV (106 dados). Em contrapartida, neste trabalho utiliza-se uma quantidade de 295 dados para o modelo DNV e 289 dados para o PCORRC. É importante destacar que quanto maior número de dados, menor o erro estatístico nas análises.

\subsection{Efeitos de variáveis no erro de modelo}

Nesta seção estudam-se como algumas variáveis do problema influenciam a precisão dos modelos semi-empíricos. As variáveis consideradas são: a resistência de escoamento mínima especificada (SMYS), a resistência ao escoamento do material $\left(\sigma_{y}\right)$, a resistência à tração mínima especificada (SMTS), a resistência à tração última do material $\left(\sigma_{u}\right)$, o diâmetro do duto (D), a espessura da parede do duto $(t)$, o fator de dilatação $(M)$ e o erro de modelo $\left(X_{M}\right)$. Na presente seção, serão analisadas algumas destas variáveis. 


\subsubsection{Erro de modelo versus resistência ao escoamento e resistência à tração última.}

A variável tensão de fluxo é calculada de forma diferente para cada modelo de pressão de ruptura. Para os modelos ASME B31G e ASME B31G modificado, utiliza-se a tensão de fluxo igual à tensão de escoamento. Nos modelos DNV RP F101 e PCORRC considera-se a tensão de fluxo igual à tensão à tração última. Na Figura 6.3a, b, c, e d tem-se o comportamento da variável erro de modelo em relação às tensões de fluxo, para os modelos: ASME B31G, B31G modificado, DNV RP F101 e PCORRC, respectivamente.

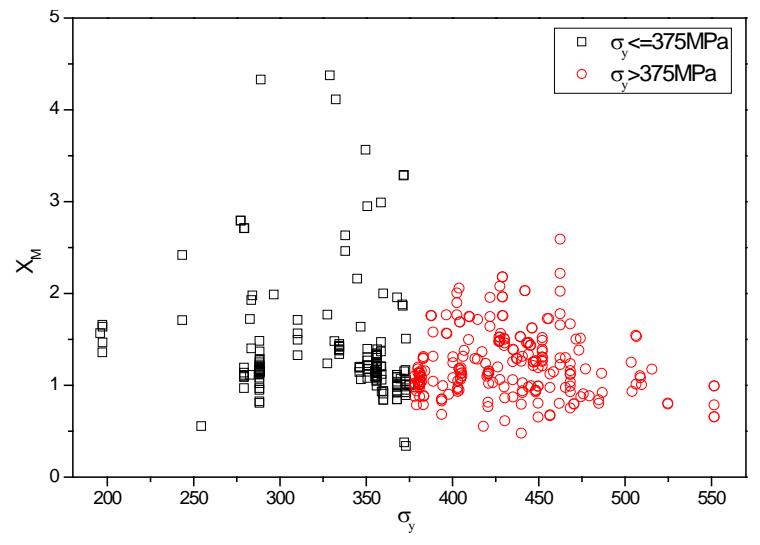

(a)

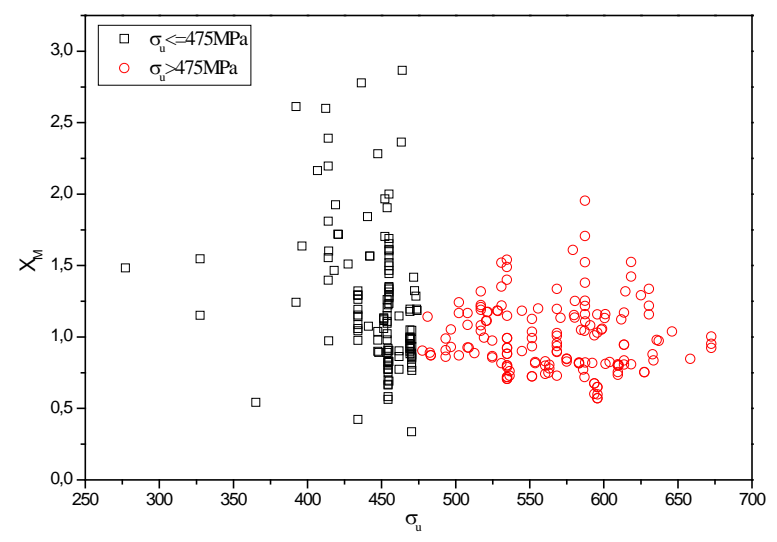

(c)

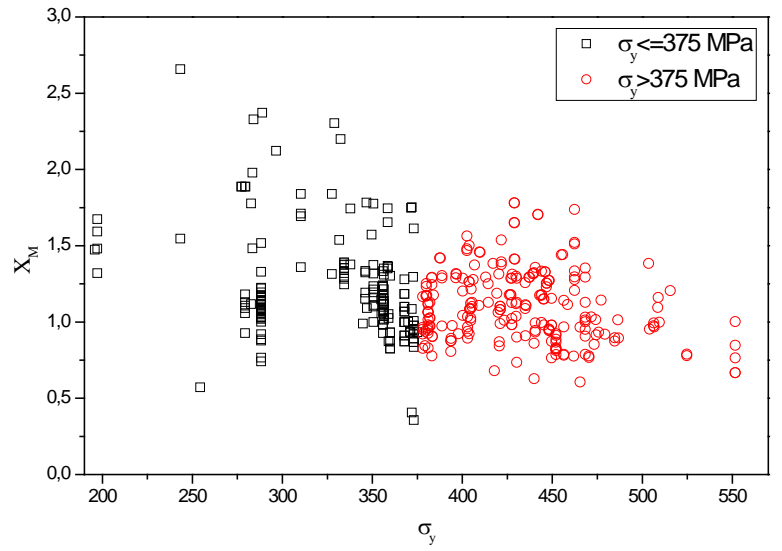

(b)

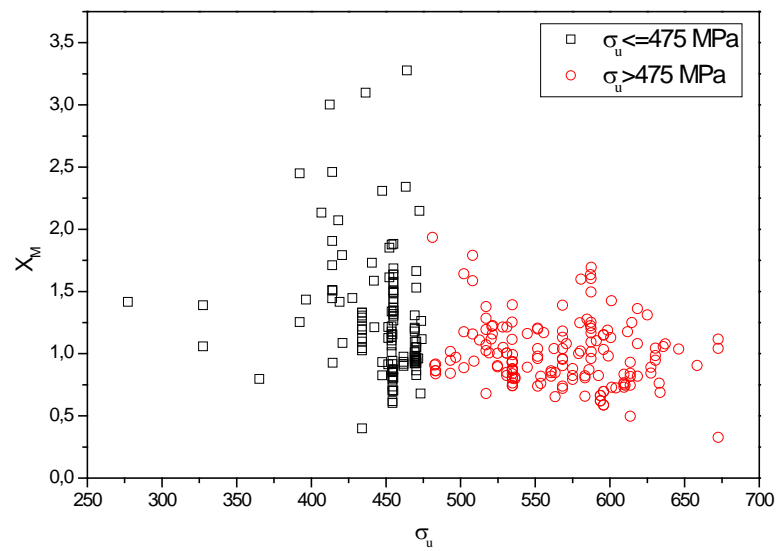

(d)

Figura 6.3. Comportamento do erro de modelo versus tensão de fluxo. (a) ASME B31G. (b) B31G modificado. (c) DNV RP F101. (d) PCORRC.

Na Figura 6.3a e Figura 6.3b, os dados se dividem em dois grupos de tensão de escoamento. Um primeiro grupo conta com 173 dados classificados para tensão de escoamento $\sigma_{y} \leq 375 \mathrm{MPa}$. O segundo contém 232 dados obtidos a partir da classificação considerando 
$\sigma_{y}>375 \mathrm{MPa}$. Após esta classificação, obtêm-se as medidas estatísticas para cada conjunto de dados com respeito à variável erro de modelo, conforme Tabela 6.6.

Tabela 6.6. Medidas estatísticas para a variável erro de modelo.

\begin{tabular}{ccccc}
\hline & \multicolumn{2}{c}{ B31G* $^{*}$} & \multicolumn{2}{c}{ B31G Modificado* } \\
$X_{M}$ & $\sigma_{y} \leq 375$ & $\sigma_{y}>375$ & $\sigma_{y} \leq 375$ & $\sigma_{y}>375$ \\
\hline$\mu$ & 1,382 & 1,219 & 1,228 & 1,096 \\
$\sigma$ & 0,640 & 0,358 & 0,353 & 0,229 \\
$\operatorname{COV}(\%)$ & 46,290 & 29,390 & 28,763 & 20,885 \\
\hline
\end{tabular}

*As unidades utilizadas para as tensões são em MPa.

Na Tabela 6.6 os dados apresentados descrevem o comportamento da variável erro de modelo para as diferentes classificações realizadas. No modelo B31G para tensões de escoamento $\sigma_{y} \leq 375 \mathrm{MPa}$, verifica-se que os dados da variável erro de modelo são conservadores. Isto acontece porque seu valor médio (1,382) é bem maior que a unidade. Também pode ser visto uma maior dispersão em relação ao tamanho da amostra, pois seu COV (46,290\%) é grande, conforme Figura 6.3a. Para $\sigma_{y}>375 \mathrm{MPa}$, os dados estatísticos para a variável erro de modelo encontram-se menos dispersos em relação ao tamanho da amostra (29,390\%) e são menos conservadores, pois seu valor médio $(1,219)$ é mais próximo da unidade.

Com relação ao B31G modificado para tensões de escoamento $\sigma_{y} \leq 375 M P a$, os dados da variável erro de modelo são conservadores, tendo em vista que seu valor médio $(1,228)$ é maior que a unidade. Este modelo apresenta uma maior dispersão, pois seu COV (28,763\%) é grande. Considerando $\sigma_{y}>375 \mathrm{MPa}$, verifica-se que os dados estatísticos para a variável erro de modelo encontram-se menos dispersos (20,885\%) e são menos conservadores, pois seu valor médio $(1,096)$ é mais próximo da unidade.

Comparando os dois modelos mencionados anteriormente, conclui-se que o B31G é o mais conservador, pois as médias do erro de modelo são maiores que as obtidos para o modelo B31G modificado. 
Para a Figura 6.3c e Figura 6.3d, os dados podem ser divididos em dois grupos de tensão à tração última. O primeiro grupo possui 132 dados obtidos a partir da classificação para tensão à tração última $\sigma_{u} \leq 475 \mathrm{MPa}$. O segundo grupo conta com 163 dados obtidos a partir da classificação dos dados para $\sigma_{u}>475 \mathrm{MPa}$. A partir desta classificação, são determinadas as medidas estatísticas para cada conjunto de dados com respeito à variável erro de modelo, conforme Tabela 6.7.

Tabela 6.7. Medidas estatísticas para a variável erro de modelo.

\begin{tabular}{|c|c|c|c|c|}
\hline \multirow{2}{*}{$X_{M}$} & \multicolumn{2}{|c|}{ DNV RP F101* } & \multicolumn{2}{|c|}{ PCORRC* } \\
\hline & $\sigma_{u} \leq 475$ & $\sigma_{u}>475$ & $\sigma_{u} \leq 475$ & $\sigma_{u}>475$ \\
\hline$\mu$ & 1,230 & 0,988 & 1,264 & 0,991 \\
\hline$\sigma$ & 0,477 & 0,237 & 0,490 & 0,257 \\
\hline COV (\%) & 38,780 & 24,018 & 38,778 & 25,900 \\
\hline
\end{tabular}

*As unidades utilizadas para as tensões são em Mpa.

Na Tabela 6.7 os dados descrevem o comportamento da variável erro de modelo para as diferentes classificações realizadas. No modelo DNV RP F101, para tensões à tração última $\sigma_{u} \leq 475 \mathrm{MPa}$, pode ser visto que os dados da variável erro de modelo são conservadores, pois seu valor médio $(1,230)$ é maior que a unidade. Estes dados apresentam uma maior dispersão em relação ao tamanho da amostra (38,780 \%), como observado na Figura 6.3c. Para $\sigma_{u}>475 \mathrm{MPa}$, os dados estatísticos da variável erro de modelo encontram-se menos dispersos em relação ao tamanho da amostra (24,018 \%) e os dados são menos conservadores, pois seu valor médio $(0,988)$ é próximo da unidade.

No modelo PCORRC, para tensões à tração última $\sigma_{u} \leq 475 \mathrm{MPa}$, verifica-se que os dados da variável erro de modelo são conservadores, já que o valor médio $(1,264)$ é maior que a unidade. Estes dados apresentam uma maior dispersão em relação ao tamanho da amostra (38,778\%), sendo grande em relação ao outro conjunto de dados, Figura 6.3d. Para $\sigma_{u}>475 \mathrm{MPa}$, os dados estatísticos da variável erro de modelo encontram-se menos dispersos $(25,90 \%)$ e são menos conservadores, pois seu valor médio $(0,991)$ é muito próximo da unidade. 


\subsubsection{Variável erro de modelo versus comprimento do defeito normalizado}

O comprimento do defeito normalizado é uma das variáveis importantes nos modelos de pressão de ruptura, já que representa a geometria do defeito de corrosão. Para obter o comprimento do defeito normalizado, deve-se aplicar a seguinte equação:

$$
L_{n}=\frac{L}{\sqrt{D t}}
$$

onde $L$ é o comprimento do defeito, $t$ é a espessura da parede e $D$ é o diâmetro do duto.

Para cada modelo de ruptura é usado um critério que determina se o comprimento do defeito normalizado é curto ou longo, para assim depois determinar a pressão de falha. Para um estudo detalhado, é realizada uma análise do comportamento da variável erro de modelo versus comprimento do defeito normalizado. Após obtidas estas variáveis, devem-se plotar os dados em um gráfico para cada modelo de pressão de ruptura e observar seu comportamento, conforme Figura 6.4.

Na Figura 6.4 pode-se observar que o comportamento do defeito normalizado apresenta duas tendências, que podem ser analisadas separadamente, considerando defeitos curtos e longos. Na Figura 6.4a, para comprimentos do defeito normalizado entre $0 \leq L_{n}<5$, os dados encontram-se agrupados. Para valores de $L_{n} \geq 5$ tem-se maior dispersão, e a variável erro de modelo apresenta comportamento crescente em relação ao comprimento do defeito normalizado.

Para a Figura 6.4b, c e d tem-se que para comprimento do defeito normalizado entre $0 \leq L_{n}<2,5$ os dados são mais próximos, apresentando pouca dispersão. Para valores de $L_{n} \geq 2,5$ os dados apresentam maior dispersão e seu comportamento é crescente em relação à variável erro de modelo. 


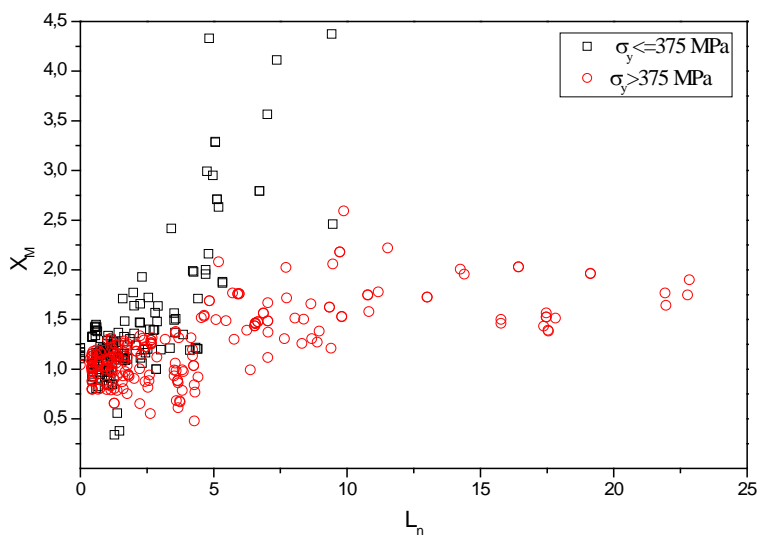

(a)

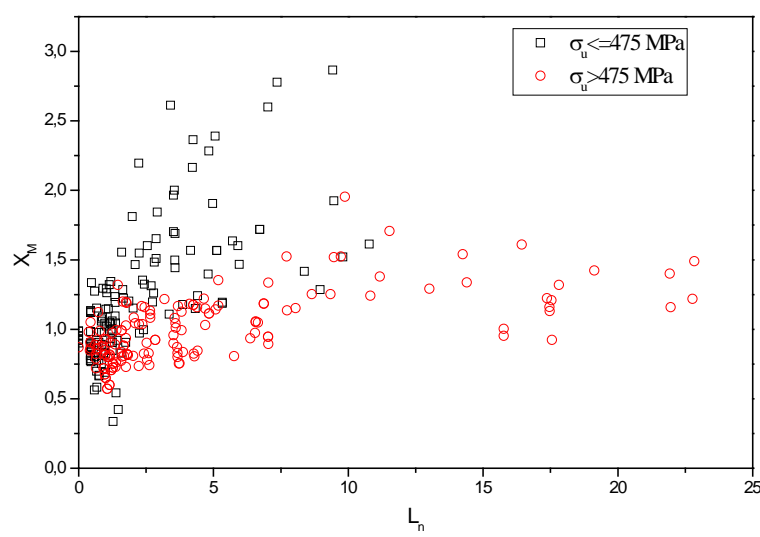

(c)

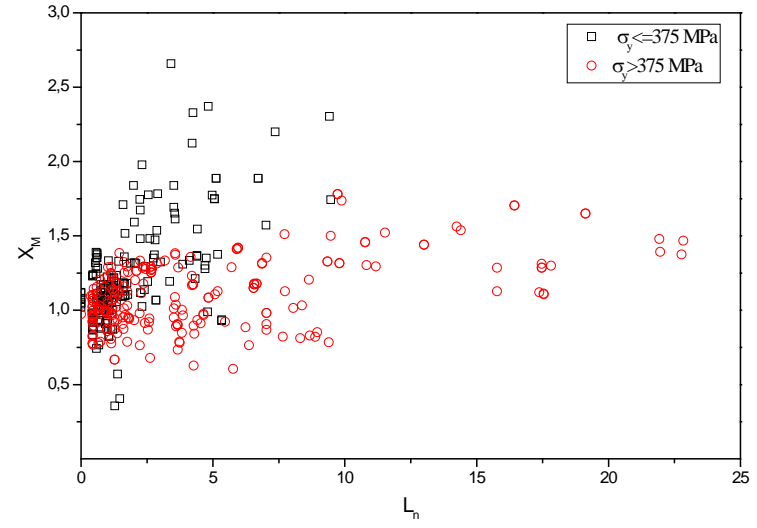

(b)

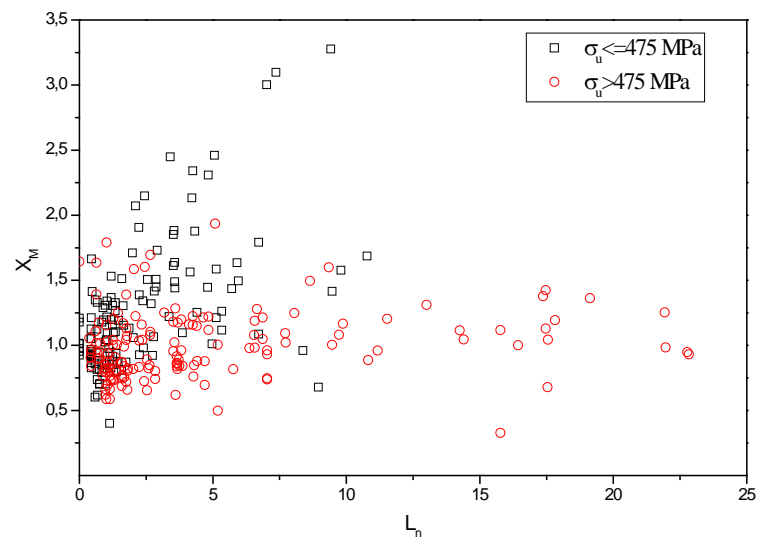

(d)

Figura 6.4. Comportamento da variável erro de modelo versus comprimento do defeito normalizado. (a) ASME B31G. (b) B31G modificado. (c) DNV-RP-F101. (d) PCORRC.

Embora a quantidade de dados utilizados por Zhou e Huang (2012) tenha sido menor que aqueles utilizados na presente pesquisa, no gráfico da variável erro do modelo versus comprimento normalizado eles apresentam comportamento semelhante, conforme Figura 6.5.

Na Figura 6.5d, observa-se que os dados apresentam um comportamento diferente aos encontrados na Figura 6.4d. Isto pode ser explicado devido aos poucos dados utilizados por Zhou e Huang (2012), que utilizam 68 dados para estas análises. Na Figura 6.4d o gráfico é realizado com 289 dados de defeitos de corrosão real. Destaca-se que com uma maior base de dados pode-se melhor representar a tendência dos dados no gráfico erro de modelo versus comprimento normalizado. 


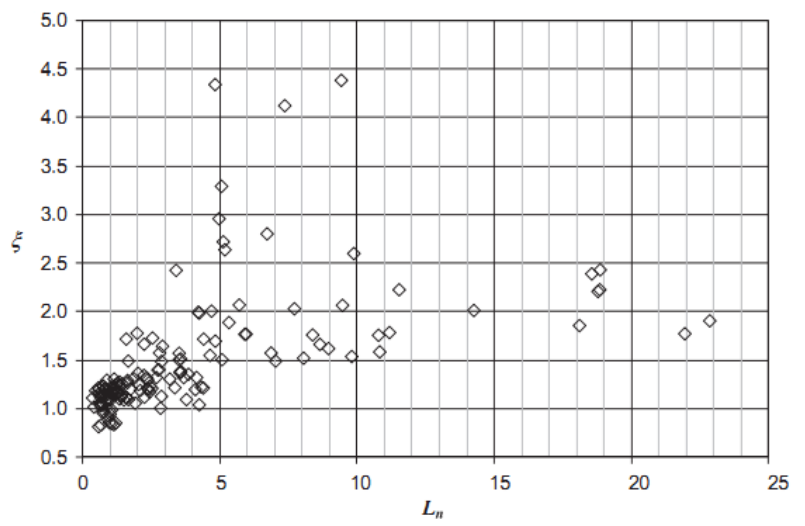

(a)

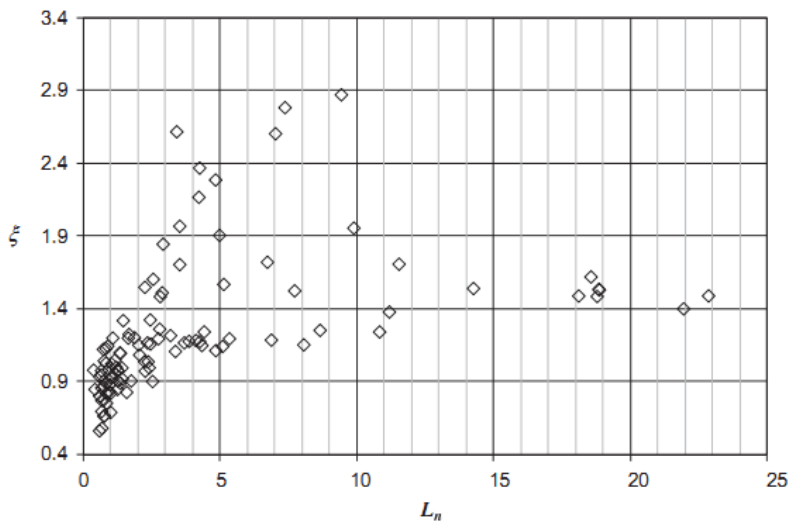

(c)

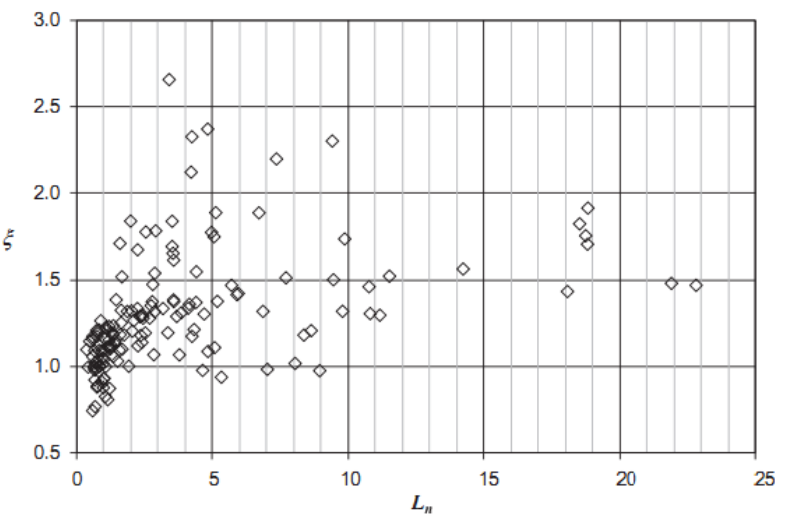

(b)

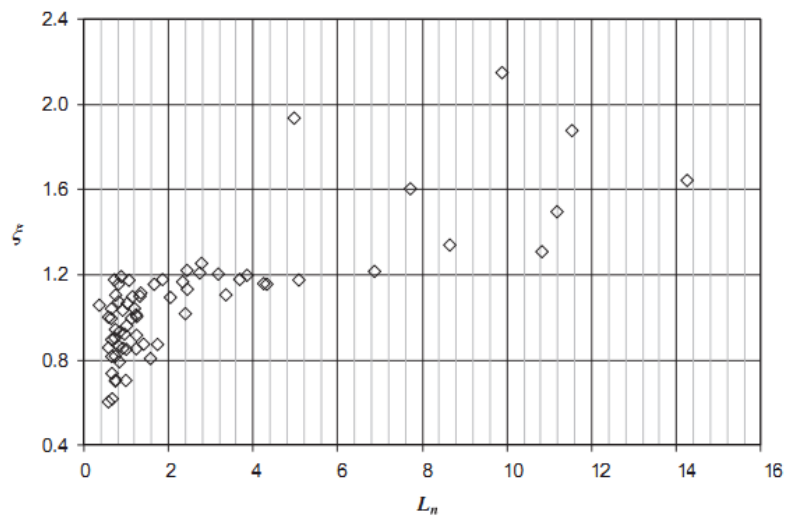

(d)

Figura 6.5. Comportamento da variável erro de modelo versus comprimento do defeito normalizado. (a) ASME B31G. (b) B31G modificado. (c) DNV-RP-F101. (d) PCORRC [Zhou; Huang, 2012].

Na seção 6.4.1, realizou-se uma classificação dos dados para tensões de escoamento $\sigma_{y} \leq 375 \mathrm{MPa}$ e $\sigma_{y}>375 \mathrm{MPa}$, sendo também classificadas para tensões à tração última de $\sigma_{u} \leq 475 \mathrm{MPa}$ e $\sigma_{u}>475 \mathrm{MPa}$. Da mesma forma a Figura 6.6, apresenta uma análise de regressão para os dados de tensão de escoamento $\sigma_{y}>375 \mathrm{MPa}$ e para tensões à tração última $\sigma_{u}>475 \mathrm{MPa}$, já que eles apresentam um comportamento menos conservador e uma tendência no conjunto dos dados. Esta análise é realizada para descrever o comportamento da variável erro de modelo versus comprimento do defeito normalizado. 


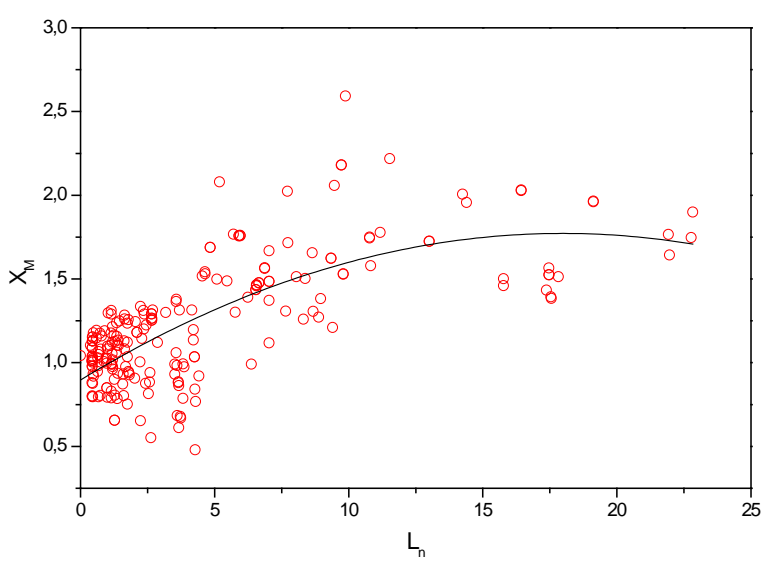

(a)

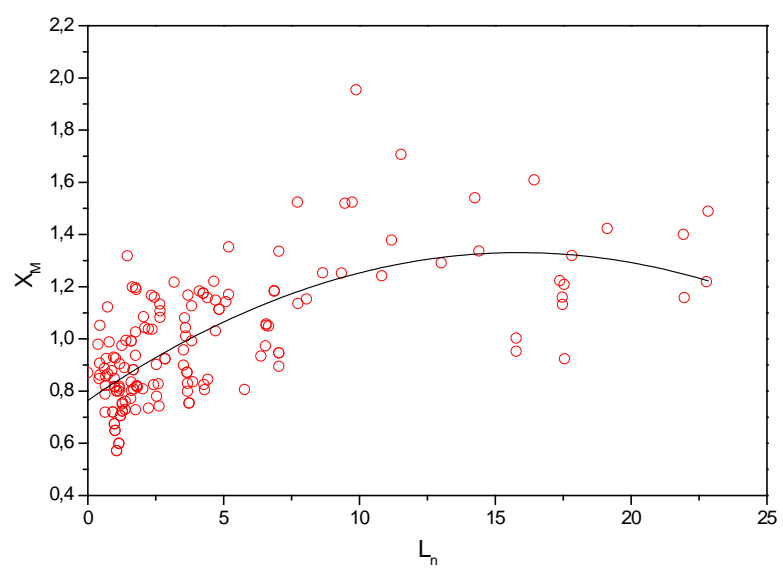

(c)

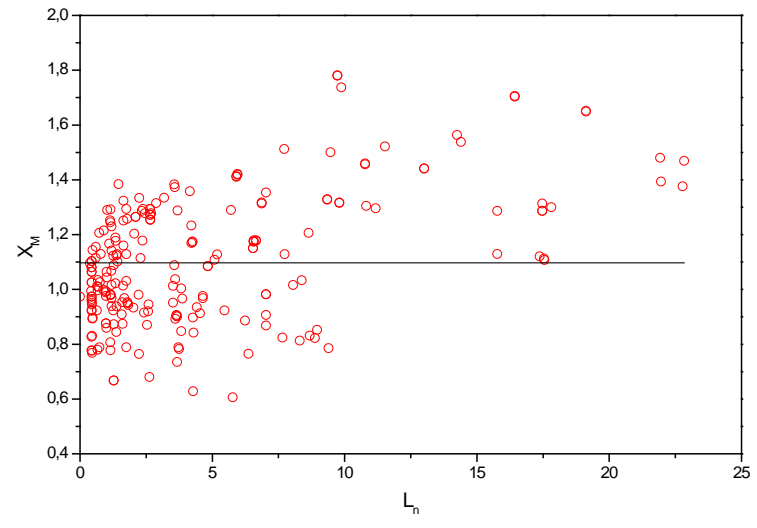

(b)

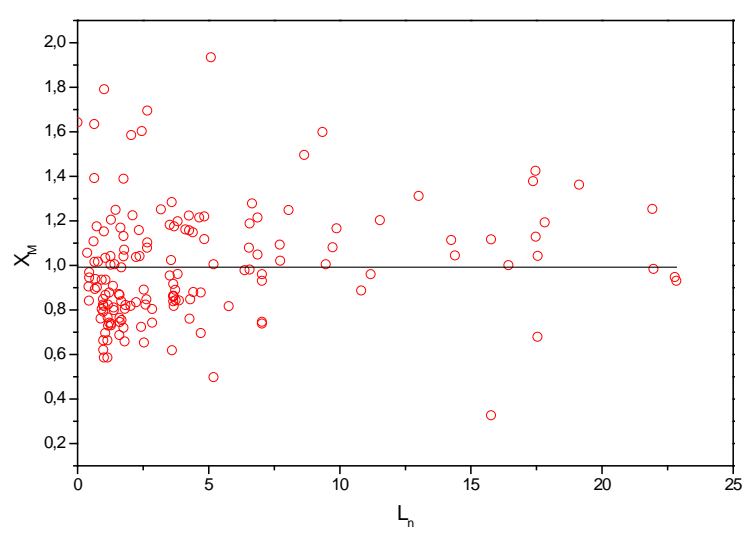

(d)

Figura 6.6. Regressão não-linear para erro de modelo versus comprimento do defeito normalizado. (a) ASME B31G. (b) B31G modificado. (c) DNV RP F101. (d) PCORRC.

Para os modelos ASME B31G e DNV RP F101 (Figura 6.6a e c), uma clara tendenciosidade é observada, para o comportamento da variável erro de modelo versus comprimento normalizado. Já para os modelos ASME B31G modificado e PCORRC, uma tendência não foi identificada.

A função que melhor representa o comportamento dos dados tem forma parabólica:

$$
X_{M}\left(L_{n}\right)=a+b \cdot\left(L_{n}\right)+c \cdot\left(L_{n}\right)^{2}
$$

onde os coeficientes do modelo não-linear utilizado encontram-se na Tabela 6.8. A Equação (6.2), juntamente com os coeficientes apresentados na Tabela 6.8, resultou em um bom ajuste de regressão, já que $\mathrm{R}^{2}$ não foi baixo apesar de se tratar de dados experimentais. 
Tabela 6.8. Coeficientes da regressão não-linear obtidos para o modelo ASME B31G e DNV RP F101.

\begin{tabular}{lll}
\hline & ASME B31G & DNV-RP-F101 \\
\hline$a$ & 0,89703 & 0,76452 \\
$b$ & 0,09734 & 0,07127 \\
$c$ & $-0,00271$ & $-0,00224$ \\
$\mathrm{R}^{2}$ & 0,5139 & 0,4635 \\
\hline
\end{tabular}

\subsubsection{Análise de regressão não-linear múltipla}

Dentre as variáveis que afetam significativamente o comportamento do erro de modelo, duas tem variação contínua ao longo do tempo, devido ao avanço da corrosão: a profundidade e o comprimento do defeito. Portanto, é importante saber como o erro de modelo se comporta em função destas duas variáveis. Desta maneira, optou-se pela realização de uma análise de regressão não-linear múltipla do erro de modelo versus profundidade relativa e comprimento normalizado do defeito. A análise de regressão não-linear múltipla é realizada apenas para a base de dados do modelo DNV RP F101. Será utilizado o modelo DNV, porque foi o modelo mais preciso e com resultados menos conservadores.

Para captar a relação que existe entre a variável erro de modelo e as duas variáveis

independentes ( $d / t$ e $L_{n}$ ), é necessária uma função que relacione não linearmente as variáveis utilizadas nesta análise:

$$
X_{M}=\frac{1}{a+b \cdot\left(\frac{d}{t}\right)^{c}}+e \cdot L_{n}+f \cdot\left(L_{n}\right)^{2}+g \cdot\left(\frac{d}{t}\right) \cdot\left(L_{n}\right) \text {, }
$$

onde as constantes $a, b, c, e, f, g$ encontram-se na Tabela 6.9. 
Tabela 6.9. Coeficientes do modelo de regressão múltipla.

\begin{tabular}{cc}
\hline Constante & Valor \\
\hline$a$ & 1,2517 \\
$b$ & 1,4442 \\
$c$ & 9,66252 \\
$e$ & 0,04278 \\
$f$ & $-0,00155$ \\
$g$ & 0,03407 \\
\hline
\end{tabular}

Estes coeficientes foram obtidos pelo método de mínimos quadrados. Esta análise de regressão múltipla apresenta um coeficiente de determinação pequeno de $R^{2}=0.47$. Isto significa que $47 \%$ da variação do erro de modelo em função de $d / t$ e $L_{n}$ é descrita pelo modelo de regressão. O coeficiente de determinação é usado como medida de qualidade do ajuste, embora o fato dele ser pequeno não indique que o ajuste seja ruim. Nem sempre a função de ajuste adotada é a mais adequada, já que para problemas físicos complexos é difícil encontrar um modelo que represente totalmente o comportamento dos dados.

O plano de regressão para o modelo (Equação (6.3)) que descreve a relação entre as variáveis $d / t, L_{n}, X_{M}$ encontra-se plotada com os dados utilizados nesta análise, conforme Figura 6.7.

Na Figura 6.7a, observa-se que para variações da razão $d / t$ entre 0,4 e 0,8 tem-se uma variação muito menor em $X_{M}$ quando $L_{n}=2$ do que quando $L_{n}=22$. 


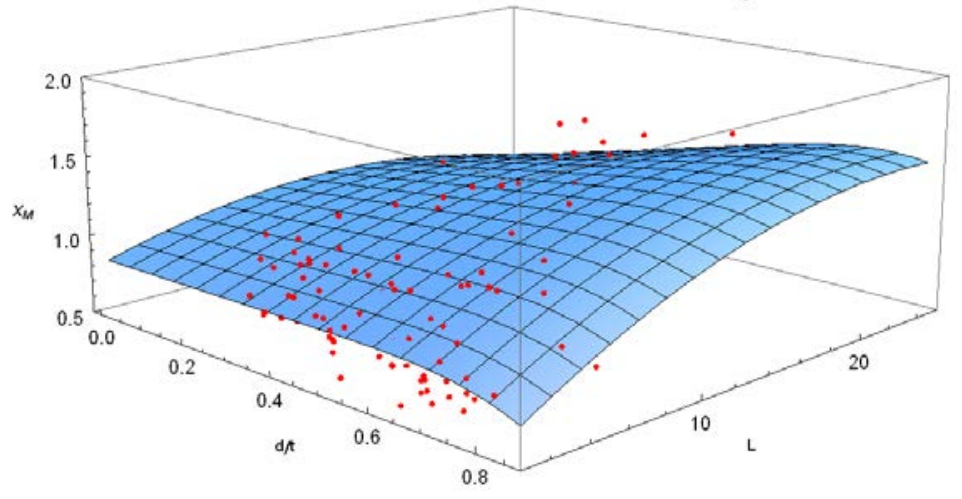

(a)

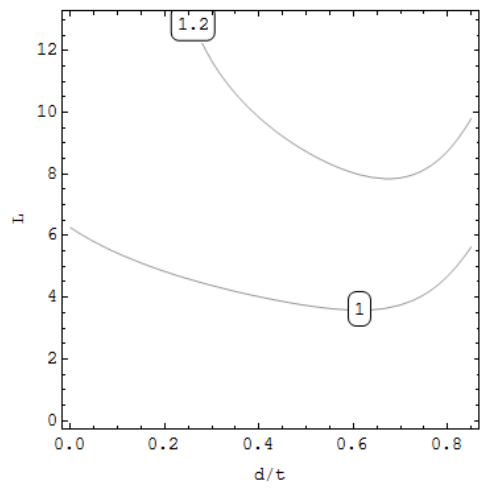

(b)
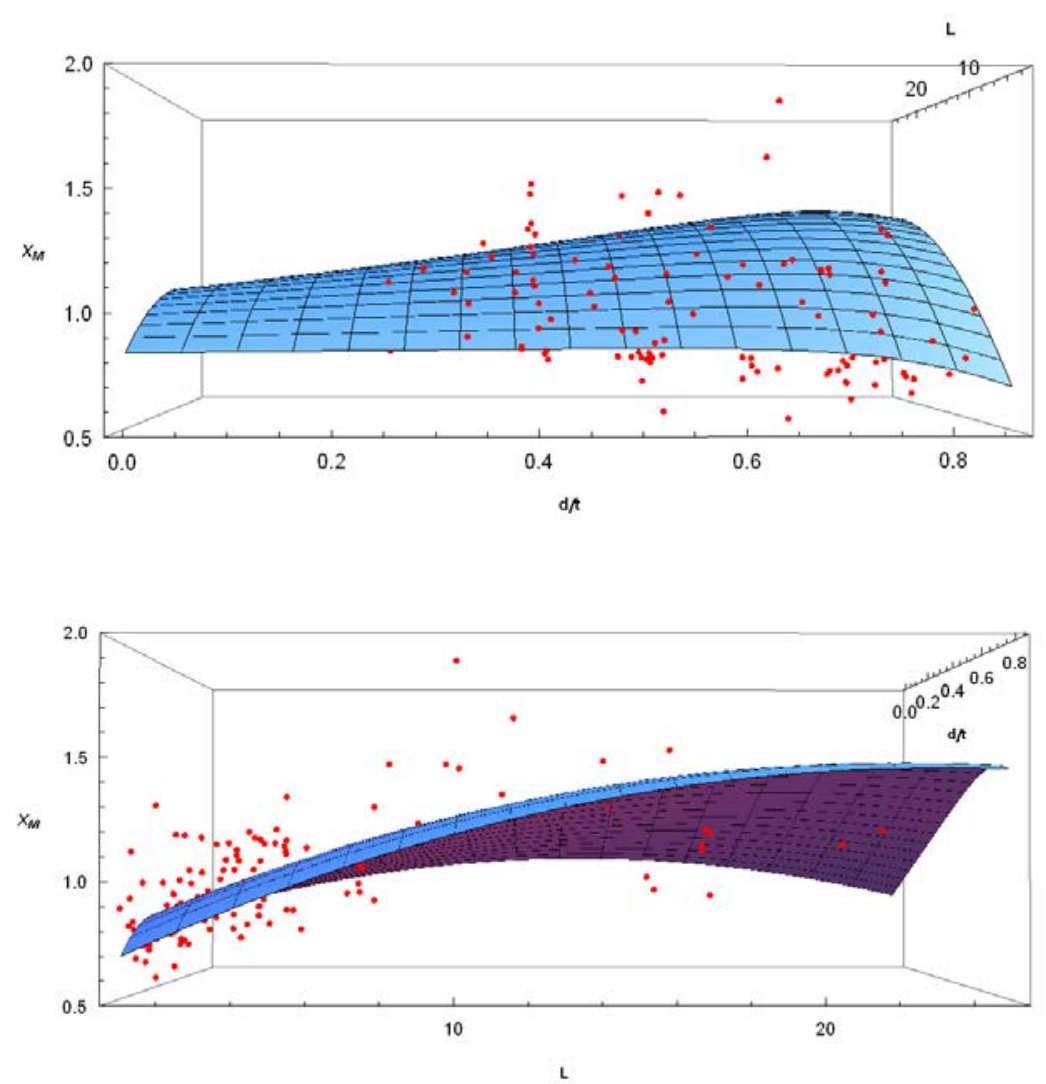

(c)

Figura 6.7. Análise de regressão não-linear múltipla. (a) Vista oblíqua do modelo de regressão. (b) Gráfico de curvas de nível. (c) Vistas laterais. 


\subsubsection{Modelos de regressão não lineares para erro de modelo versus profundidade relativa do defeito.}

A variável erro de modelo ( $X_{M}$ ) é obtida avaliando e comparando as pressões de ruptura medidas em ensaios de ruptura em dutos a escala real (full-scale), com resultados obtidos a partir dos modelos de predição de ruptura.

Como a corrosão evolui com o tempo, a profundidade dos defeitos aumenta. Assim, para fazer previsões da segurança de um duto ao longo do tempo, é necessário saber como a incerteza do modelo evolui com o aumento da profundidade do defeito. Sabe-se também que o aumento da profundidade é mais deletério para a segurança do duto do que o aumento do comprimento do defeito.

Quando a razão $d / t$ está próxima da unidade, significa que o duto se encontra próximo da falha, podendo apresentar ruptura ou vazamento. Quando a razão $d / t$ é próxima de zero, significa que o defeito de corrosão é incipiente, com pequeno potencial de causar falha do duto. Neste estudo será utilizado um modelo de regressão não linear para todos os modelos, já que eles apresentam quase o mesmo comportamento dos dados. Os modelos de ajuste não linear utilizados neste trabalho, foram obtidos a partir de testes com funções que melhor representasse o comportamento dos dados. Portanto, o modelo não linear para representar o comportamento da variável erro do modelo em função da razão $d / t$, é dado por:

$$
X_{M i}=\frac{1}{a+b \cdot(d / t)^{c}}
$$

\subsubsection{Modelo DNV RP F101}

Nesta seção é apresentado um estudo do comportamento da variável erro de modelo com a razão $d / t$, para o modelo da norma DNV RP F101. A quantidade de dados utilizada é de 311 defeitos reais (Tabela 6.2). Na Figura 6.8 é possível observar uma relação de dependência 
entre estas variáveis, com uma variação crescente do erro de modelo $\left(X_{M}\right)$ em função da razão $d / t$. O gráfico corresponde aos dados disponíveis para o estudo das variáveis (Tabela 6.2). Devido à dispersão dos dados para razão $d / t$ próxima da unidade, será utilizada uma recomendação prática da DNV RP F101, segundo a qual a profundidade do defeito não pode exceder $85 \%$ da espessura da parede, ou seja, $d / t \leq 0,85$. Desta maneira, após filtrar a base de dados, obtém-se 295 dados de defeitos reais, conforme Tabela 6.3. Em seguida é realizada uma análise de regressão não-linear, já que uma regressão linear não descreve as tendências observadas. A análise é apresentada na Figura 6.9a. A Figura 6.9b mostra a variação dos resíduos em relação à razão $d / t$.

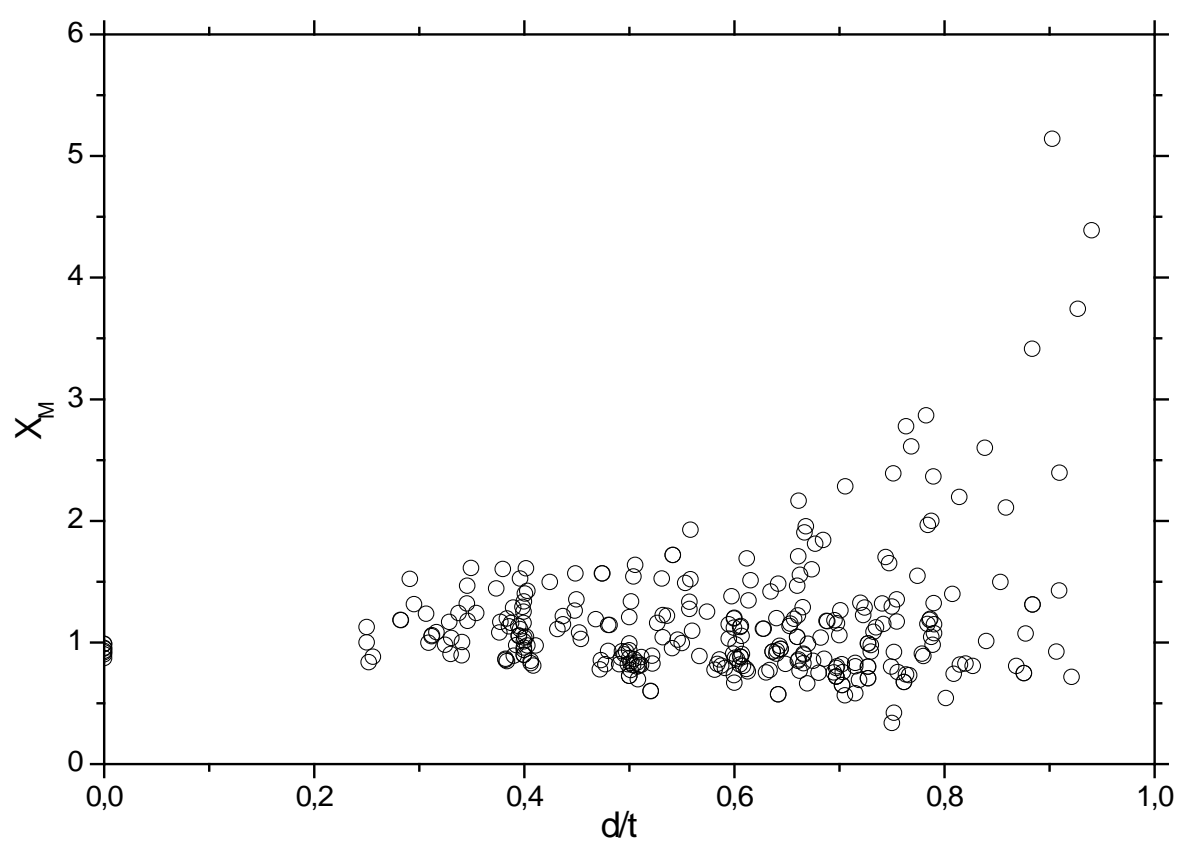

Figura 6.8 Comportamento do erro de modelo versus razão $d / t$, modelo DNV.

Na Figura 6.9a, verifica-se que a função não-linear encontrada e seu respectivo ajuste dos dados obtido pelo método dos mínimos quadrados ordinários (MQO), não descrevem o problema físico. Isto acontece porque a discrepância dos dados em relação à linha de ajuste é grande. Embora, o desvio padrão seja pequeno $(\sigma=0.3773)$ não indica que os dados estejam próximos da média (Figura 6.9b). Nestas situações, é difícil encontrar um modelo de regressão que se ajuste ao comportamento dos dados, já que apresentam grandes variações. Os 
coeficientes do modelo de regressão não-linear utilizados (Equação (6.4)) são apresentados na Tabela 6.10.

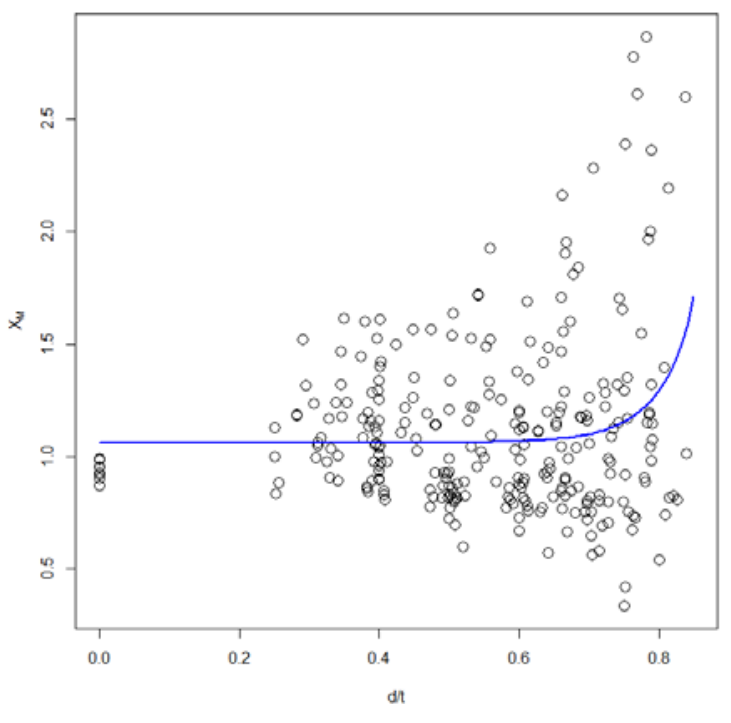

(a)

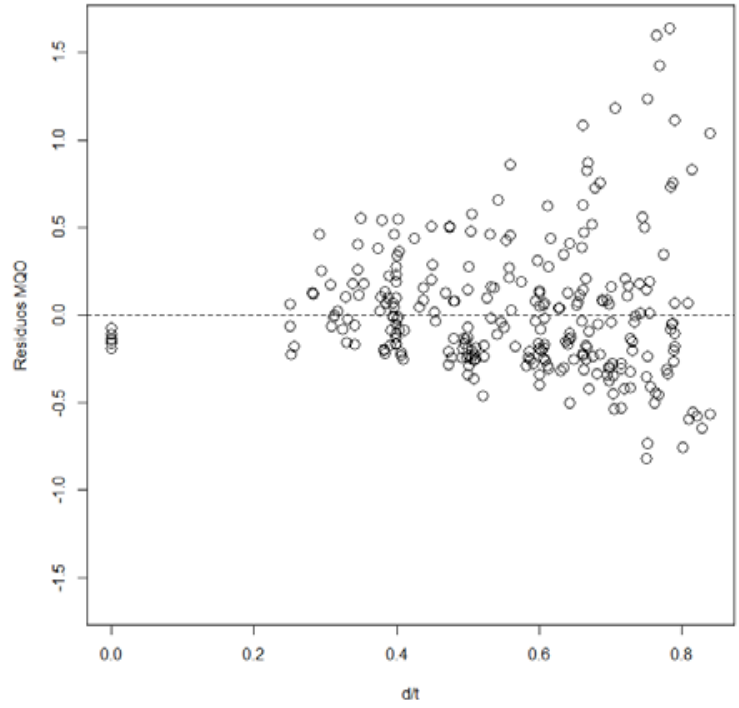

(b)

Figura 6.9. (a) Regressão não-linear da variável $X_{M}$ versus razão $d / t$.

(b) Variação dos resíduos em relação à razão $d / t$.

Tabela 6.10. Coeficientes ajustados do modelo não-linear, DNV RP F101.

\begin{tabular}{ccc}
\hline Coeficientes & MQO & MQP \\
\hline$a$ & 0,94173 & 0,93411 \\
$b$ & $-2,74745$ & $-5,06091$ \\
$c$ & 12,53682 & 15,58201 \\
\hline
\end{tabular}

Considerando o modelo não-linear, é possível encontrar alguns problemas que estão envolvidos nos dados. Analisando a Figura 6.9b, tem-se que o desvio padrão da variável erro de modelo em relação à razão $d / t$ não é uniforme. A não uniformidade gera um tipo de problema que é possível encontrar em áreas das ciências sociais, economia e em aplicações da ciência biológica. Tal problema apresenta um comportamento de tendência crescente nos erros, indicando a violação da hipótese de homogeneidade da variância, que neste caso ocorre em forma de funil ou megafone. Devido à violação do pressuposto de homoscedasticidade, o gráfico dos resíduos do modelo é usado para verificar se há presença de heteroscedasticidade. 
Na presença de heteroscedasticidade, devem ser tomadas medidas corretivas, já que trata-se de um problema estatístico que deve ser solucionado. A heterocedasticidade é um problema físico que ocorre quando o modelo de regressão utilizado apresenta variâncias não iguais para as observações. A variância não constante para as observações pode ser causada por: coleta inadequada de dados, incorreta transformação de dados, dados atípicos, assimetria na distribuição das variáveis, etc.

Para encontrar uma solução do problema estatístico, utilizaram-se diversos modelos de transformação Box-Cox, mas os resultados obtidos não foram satisfatórios, no sentido de se obter variância uniforme.

Para aplicar as medidas corretivas, primeiro é necessário garantir a existência da heteroscedasticidade aplicando algum dos métodos existentes. Neste trabalho, é utilizado o método gráfico, que consiste em realizar uma análise de regressão com o suposto de variância constante, e em seguida obter o valor quadrático dos resíduos estimados. Em seguida, estes valores são dispostos em um gráfico com a variável independente (Figura 6.10), observandose se os mesmos apresentam um padrão sistemático.

Na Figura 6.10, é possível confirmar a existência de heteroscedasticidade, já que o valor quadrático dos resíduos apresenta um padrão definido. Este padrão pode ser comparado com outros já estabelecidos na Figura 4.5.

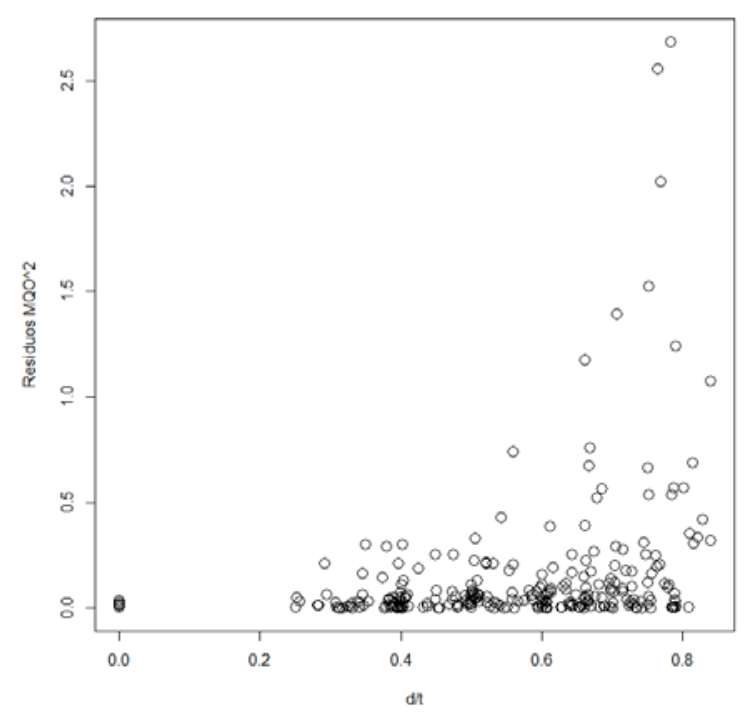

Figura 6.10. Valor quadrático dos resíduos estimados pelo método dos MQO. 
A medida corretiva para este fenômeno estatístico consiste em utilizar o método dos mínimos quadrados ponderados (MQP). Este método aplica diferentes pesos a cada observação. Estes pesos são inversamente proporcionais à variância, para assim, obter uma variância homogênea. O método MQP, é uns dos métodos diretos para corrigir a heteroscedasticidade. Aplicando o método MQP, uma primeira etapa consiste em aplicar o valor absoluto aos resíduos estimados pelo método MQO, e em seguida realizar uma análise de regressão das observações, para assim obter uma função estimada do desvio padrão, conforme Figura 6.11.

Na Figura 6.11, aplica-se um modelo de regressão não-linear às observações do desvio padrão, que descreve uma relação entre $\sigma_{i}$ e razão $d / t$. Este modelo não-linear apresenta o seguinte comportamento:

$$
\hat{s}=\frac{1}{6.368-7.286 \cdot\left(\frac{d}{t}\right)^{2.155}},
$$

onde $\hat{s}$ é a função estimada da análise de regressão do desvio padrão esperado. A partir da função estimada para o desvio padrão, obtêm-se os pesos utilizados para realizar a ponderação a cada observação, conforme Equação (4.51).

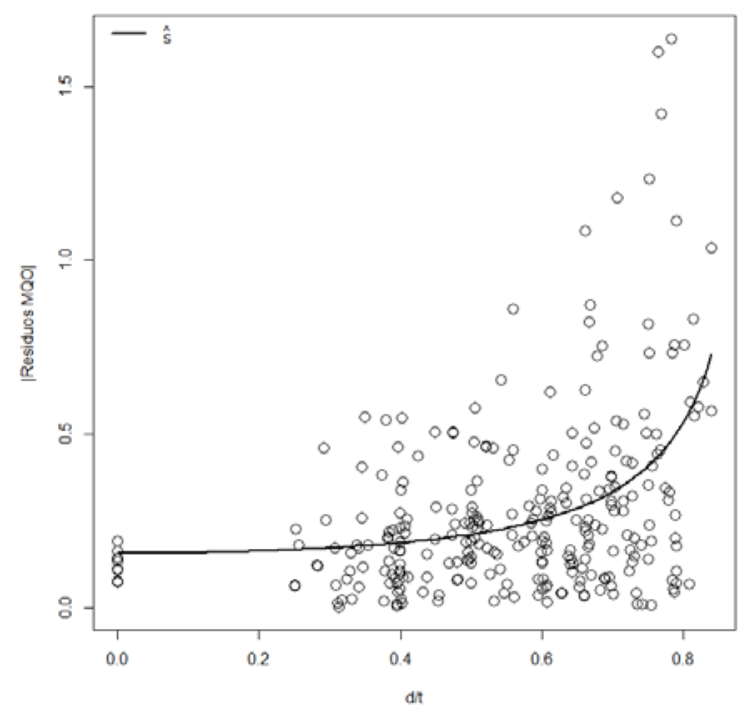

Figura 6.11. Valor absoluto do resíduo estimado pelo método MQO versus razão $d / t$. 
Segundo Gujarati e Porter (2009), o peso atribuído a cada observação é inversamente proporcional à variância. Portanto, observações que pertencem a uma população com desvio padrão grande terão ponderações relativamente pequenas. Por outro lado, para observações que pertencem a uma população com desvio padrão pequeno deve ser aplicada uma ponderação proporcionalmente grande. Os pesos que serão aplicados nas observações são apresentados na Figura 6.12.

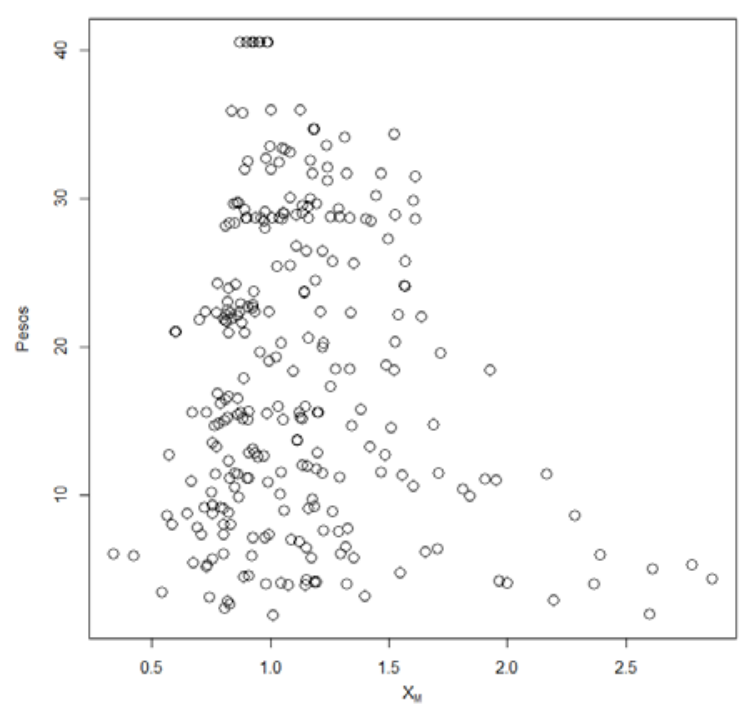

Figura 6.12. Pesos obtidos para cada observação.

Proporcionando os pesos ao método MQP, é possível realizar uma regressão não-linear e estimar os coeficientes que possuem variância mínima (Tabela 6.10).

Na Figura 6.13a, é encontrada a função do modelo de regressão não-linear, ajustado pelo método MQP. O ajuste realizado apresentou um desvio padrão $(\sigma=1,259)$ grande, que indica que os dados estão mais espalhados em comparação ao método MQO $(\sigma=0,3773)$, mas sem o fenômeno de heteroscedasticidade (Figura 6.13b). O modelo de regressão utilizado é apresentado na Equação (6.4) e seus coeficientes são apresentados na Tabela 6.10 (terceira coluna).

Na Figura 6.13b, os resíduos estimados pelo método dos MQP encontram-se distribuídos aleatoriamente em torno do zero, sem comportamento ou tendência significativa. A partir disto, conclui-se que os resultados dos resíduos estimados pelo método MQP satisfazem o suposto de homoscedasticidade do modelo não-linear. 


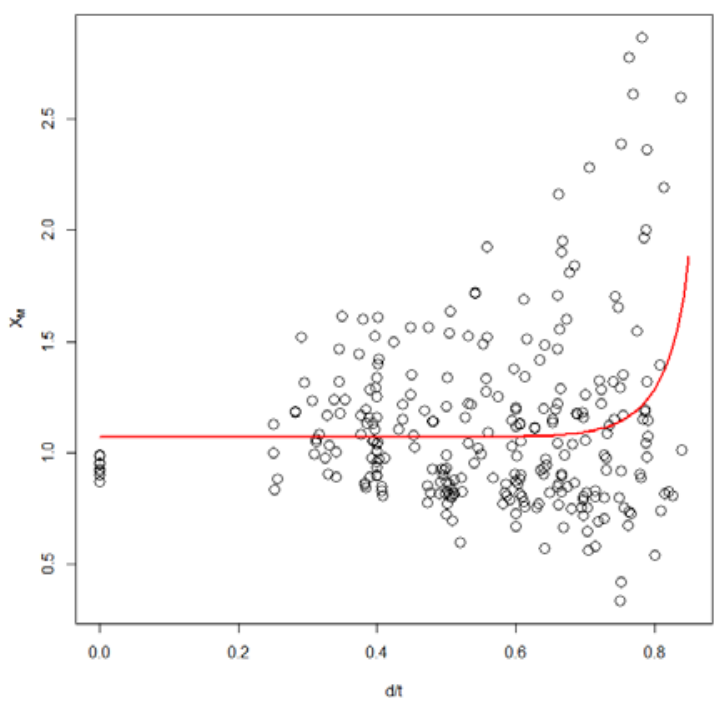

(a)

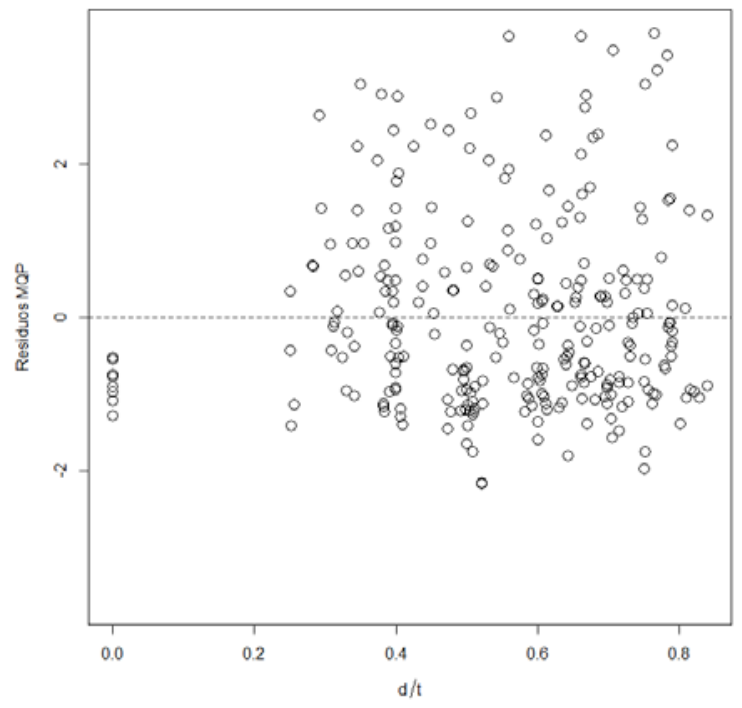

(b)

Figura 6.13. (a) Regressão não-linear das variáveis $X_{M}$ versus razão $d / t$.

(b) Variância dos erros em relação à razão $d / t$.

Finalmente, nas Figura 6.9b e Figura 6.13b, apresenta-se uma grande diferença entre os resíduos obtidos a partir dos métodos de ajuste MQO e MQP. Estes dois métodos não podem ser comparados, pois as variáveis são diferentes depois de serem aplicados os pesos. Mesmo assim, as pequenas diferenças nos coeficientes são suficientes, para obter homoscedasticidade nos resíduos (Figura 6.13b). Com a solução deste tipo de problema é possível ter uma maior confiança nos ajustes realizados.

\subsubsection{Modelo PCORRC}

A base de dados utilizada para o modelo PCORRC possui 311 dados de defeitos reais (Tabela 6.2). A partir destes dados, é possível encontrar um modelo de regressão que descreve o problema físico envolvido. A quantidade de dados de defeitos reais é apresentada na Figura 6.14 .

Os dados apresentados na Figura 6.14 correspondem à totalidade das observações disponíveis para as análises do modelo PCORRC (Tabela 6.2). Após aplicar o mesmo critério de filtragem utilizado para o modelo DNV $(d / t \leq 0,85)$ classificou-se a base de dados, obtendo-se 289 
dados de defeitos reais (Tabela 6.3). Para uma adequada modelagem, deve-se encontrar um modelo que represente o comportamento que apresentam estes dados. Portanto, realiza-se uma análise de regressão não-linear para quantificar o comportamento de $X_{M}$ com a razão $d / t$. Esta análise de regressão é apresentada na Figura 6.15a, e seus respectivos resíduos na Figura 6.15b.

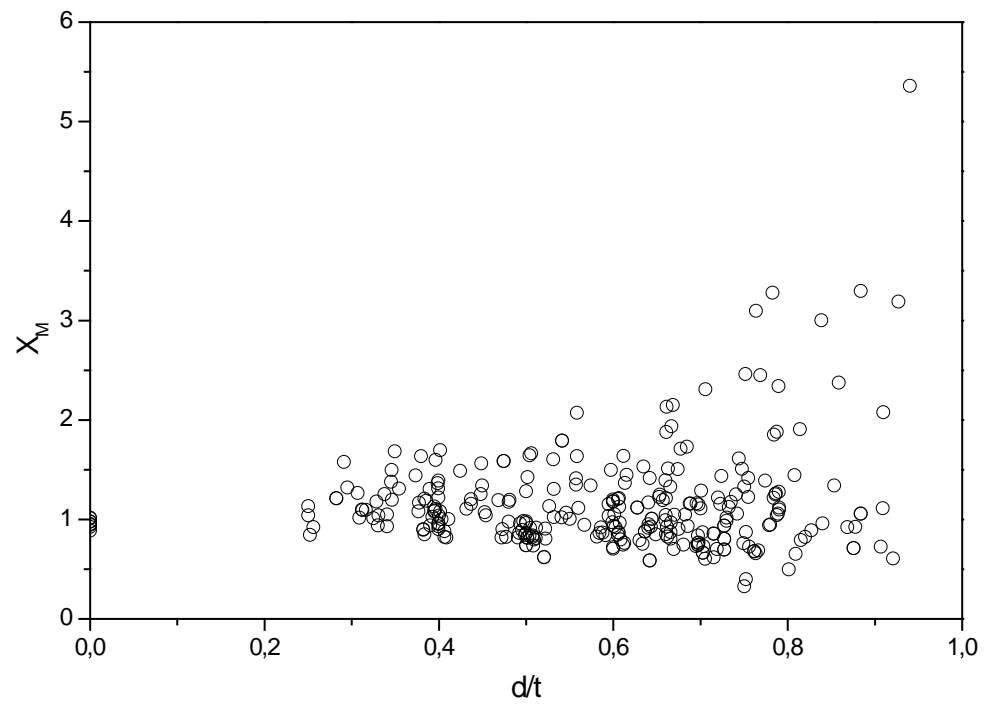

Figura 6.14. Comportamento da variável erro de modelo versus razão $d / t$, modelo PCORRC.
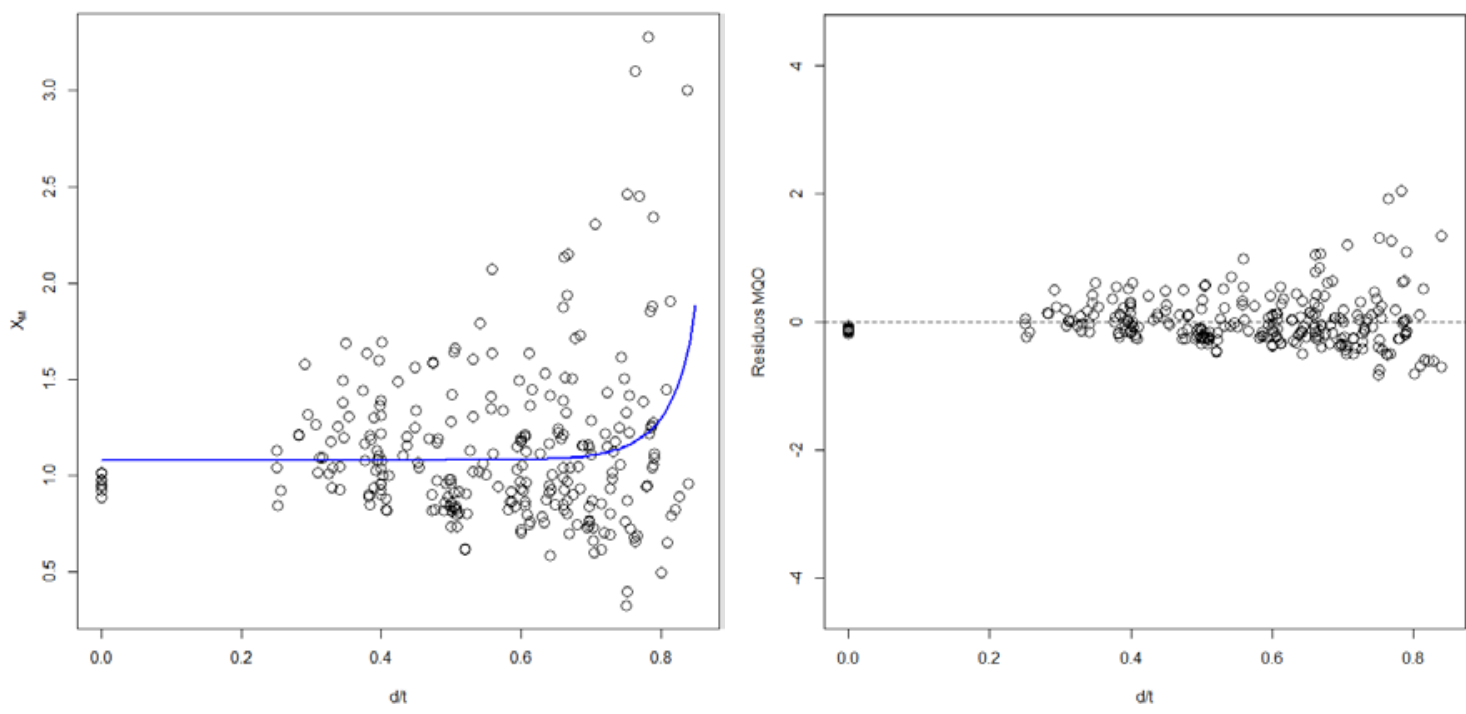

Figura 6.15. (a) Regressão não-linear das variáveis $X_{M}$ versus razão $d / t$.

(b) Variância do erro proporcional à razão $d / t$. 
Para a análise de regressão não-linear é utilizado o modelo apresentado na Equação (6.4), já que os dados apresentam um comportamento similar ao modelo DNV. Desta maneira, obtémse os coeficientes do modelo de regressão a partir do método de ajuste MQO, apresentados na Tabela 6.11. Na Figura 6.15a, o ajuste do modelo de regressão não-linear realizado, não representa completamente o problema físico. Isto acontece porque a discrepância dos dados é grande em relação à linha de ajuste. Portanto, analisando as características estatísticas da análise de regressão, pode-se dizer que o desvio padrão $(\sigma=0.3954)$ é pequeno, mas não indica que os resíduos estejam em torno da média. Isto é observado na Figura 6.15b que representa a variância em relação à variável dependente.

Na Figura 6.15b, os dados não apresentam variância uniforme, este é um problema similar ao apresentado no modelo DNV. Para verificar a presença de heteroscedasticidade é utilizado o método gráfico, conforme descrito anteriormente.

Tabela 6.11. Coeficientes ajustados da regressão não-linear, PCORRC.

\begin{tabular}{ccr}
\hline Coeficientes & \multicolumn{1}{l}{ MQO } & \multicolumn{1}{c}{ MQP } \\
\hline$a$ & 0,92442 & 0,91842 \\
$b$ & $-4,77815$ & $-7,92435$ \\
$c$ & 15,36325 & 17,9405 \\
\hline
\end{tabular}

Na Figura 6.16 é aplicado o método gráfico, podendo ser visto que o valor quadrático dos resíduos estimados pelo método MQO apresenta um comportamento crescente. Realizando uma comparação da Figura 6.16 e a Figura 4.5, constata-se que os resíduos apresentam um padrão sistemático, confirmando a presença de heteroscedasticidade.

Para contornar o problema de heteroscedasticidade, é aplicado o método dos Mínimos Quadrados Ponderados (MQP). Neste sentido, primeiramente deve-se obter uma função de regressão estimada do desvio padrão, a qual é apresentada na Figura 6.17, sendo o modelo utilizado para o ajuste dos dados: 


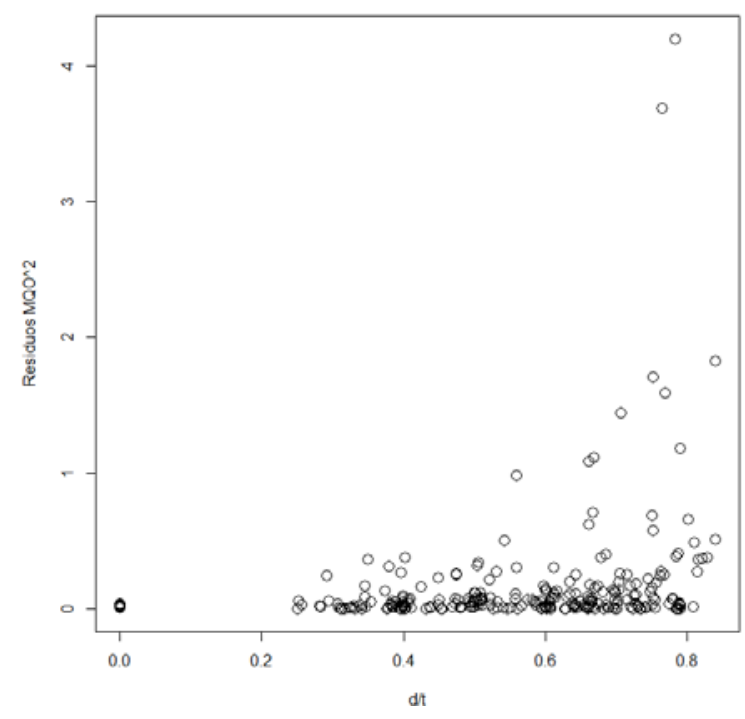

Figura 6.16. Valor quadrático dos resíduos estimados pelo método dos MQO.

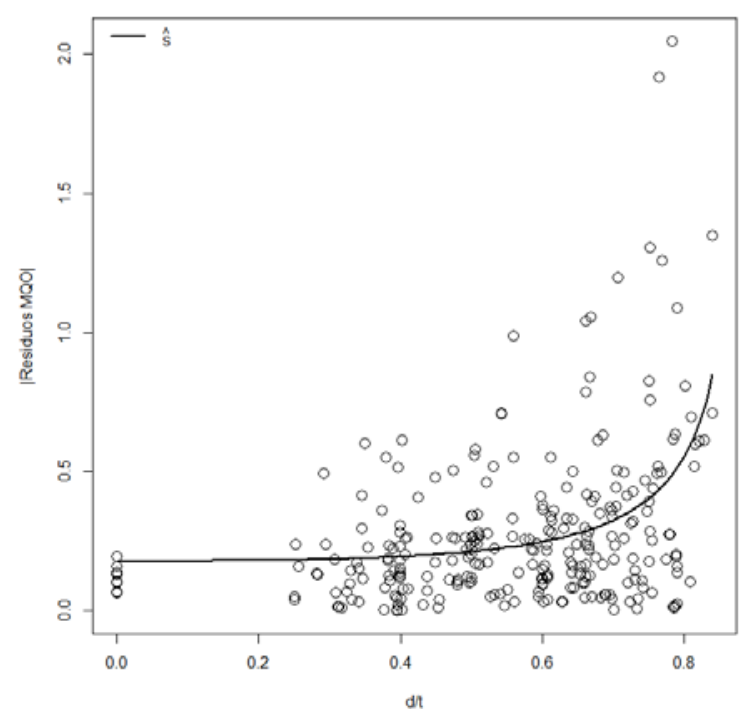

Figura 6.17. Valor absoluto do residual estimado pelo método dos MQO versus razão $d / t$.

$$
\hat{s}=\frac{1}{5.61-7.5682 \cdot\left(\frac{d}{t}\right)^{3.0653}},
$$

onde $\hat{s}$ é a função não linear do desvio padrão esperado.

Em seguida, são determinados os pesos que devem ser aplicados a cada observação, conforme Equação (4.51). A ponderação dos pesos depende da variação do erro que apresenta cada 
observação. A partir do modelo de regressão do desvio padrão, têm-se os pesos para cada observação, conforme Figura 6.18.

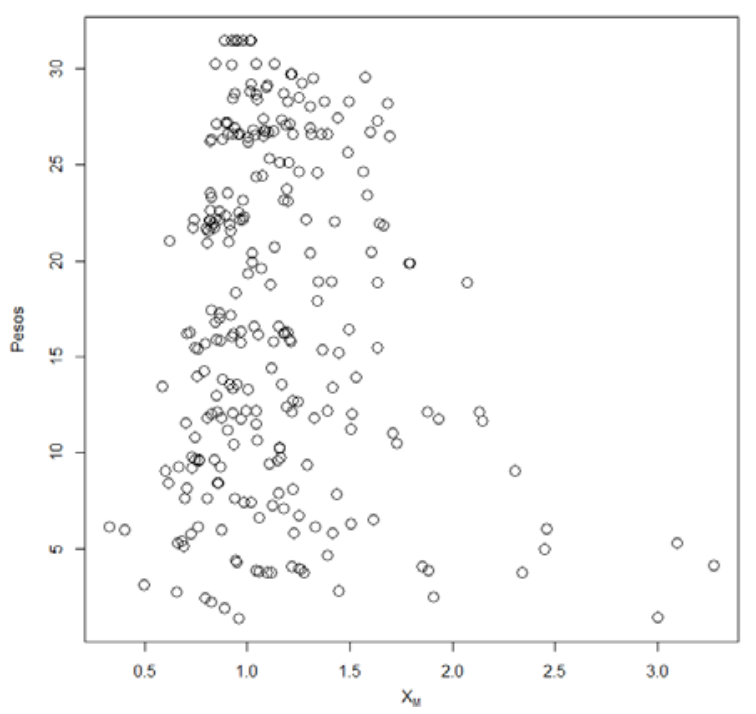

Figura 6.18. Pesos obtidos para cada observação.

Assim, proporcionando os pesos ao método MQP, é possível realizar uma análise de regressão com os dados já ponderados e assim, estimar os coeficientes que diminuem a variância. Para análise de regressão não-linear é utilizada a Equação (6.4) e os coeficientes são apresentados na Tabela 6.11.

Na Figura 6.19a, é realizada a análise de regressão não-linear, obtendo a função que melhor se ajusta aos dados pelo método MQP. Este ajuste apresenta uma grande dispersão $(\sigma=1.259)$, indicando que os dados estão mais espalhados em comparação ao método MQO $(\sigma=0.3954)$, mas sem o fenômeno de heteroscedasticidade (Figura 6.19b).

Na Figura 6.19b, os resíduos estimados pelo método MQP, encontram-se distribuídos aleatoriamente em torno do zero, tendo um comportamento ou tendência mais homogênea. Portanto, conclui-se que os resíduos estimados pelo método MQP satisfazem o suposto de homoscedasticidade do modelo não-linear. 

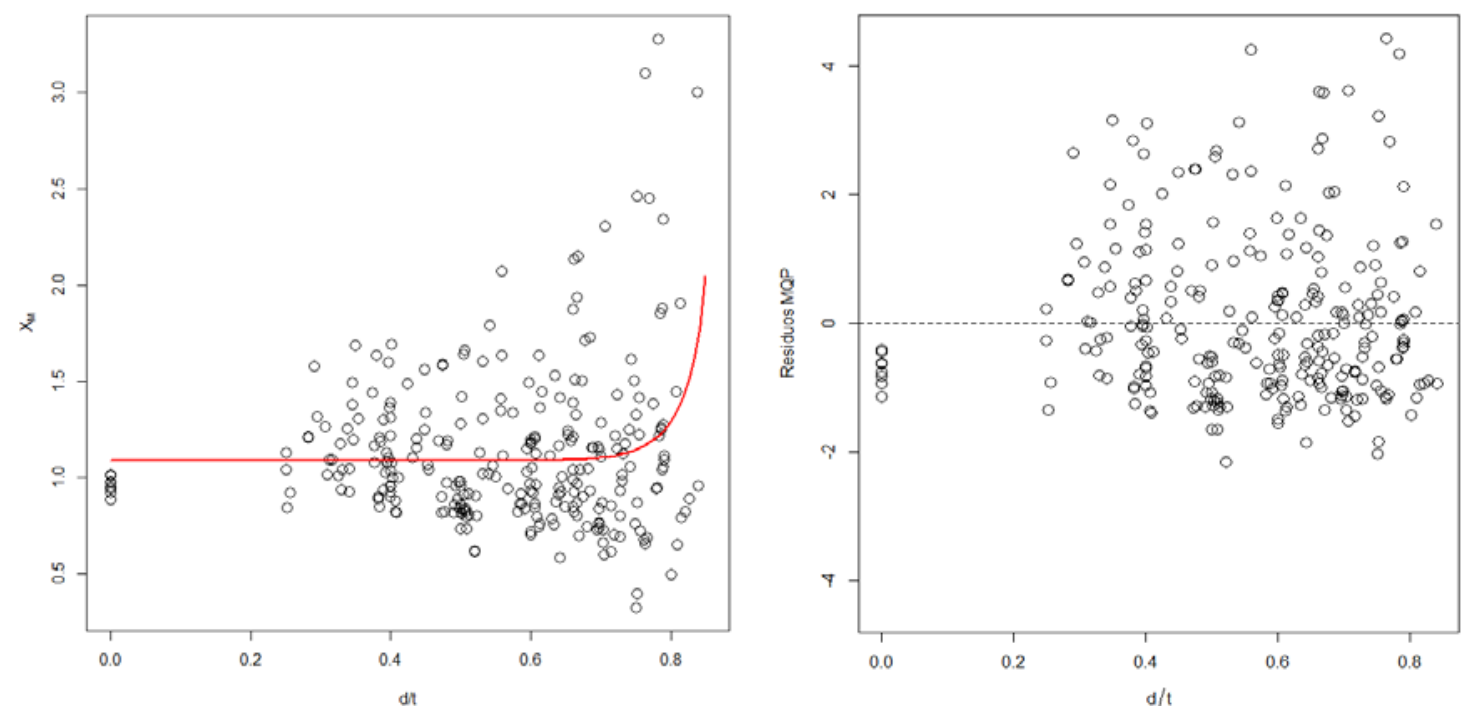

Figura 6.19. (a) Regressão não linear das variáveis $X_{M}$ versus razão $d / t$;

(b) Variância do erro uniforme proporcional à razão $d / t$.

Nas Figura 6.15b e Figura 6.19b, pode ser vista a diferença entre os resíduos dos modelos de regressão não linear ajustados pelos métodos MQO e MQP. Os coeficientes apresentam uma pequena variação que satisfazem os suposto de homoscedasticidade (Tabela 6.11).

De maneira geral, verifica-se que ambos os métodos apresentam comportamentos similares, porém, foi destacado que o método de ajuste MQO apresenta heteroscedasticidade. Isto torna o método de ajuste MQP o mais adequado para representar o problema físico dos dados estudados. É importante destacar que tais métodos não podem ser comparados, já que após a ponderação dos pesos as variáveis não são as mesmas.

\subsubsection{Modelo ASME B31G}

O modelo ASME B31G, possui na totalidade 405 dados de defeitos reais e artificiais (Tabela 6.2). O comportamento da variável erro de modelo $X_{M}$ e a razão $d / t$, são encontrados na Figura 6.20.

Para um estudo detalhado, é importante refinar a base de dados, já que a partir de certo valor da razão $d / t$, a variável erro de modelo $\left(X_{M}\right)$ começa aumentar de maneira desproporcionada. Assim, aplica-se o critério de filtragem dos dados utilizado no modelo DNV $(d / t \leq 0,85)$. A filtragem realizada ajuda a excluir os dados que possam gerar alguma 
tendência nas análises posteriores. Após a filtragem obtém-se 405 dados (Tabela 6.4), que representam o comportamento de defeitos reais e artificiais. Ressaltasse que para defeitos de corrosão com perda de espessura da parede entre $10 \%$ e $80 \%$ o duto deve ser reparado ou substituído segundo a ASME B31G. Esta limitação não contaminam os resultados devido a que o volumem de dados é muito baixo. Em seguida, deve-se encontrar um modelo que descreva a tendência dos dados. O modelo utilizado para a análise de regressão não-linear tem a forma da Equação (6.4). Esta análise de regressão não-linear é apresentada na Figura 6.21a com seus respectivos resíduos na Figura 6.21b.

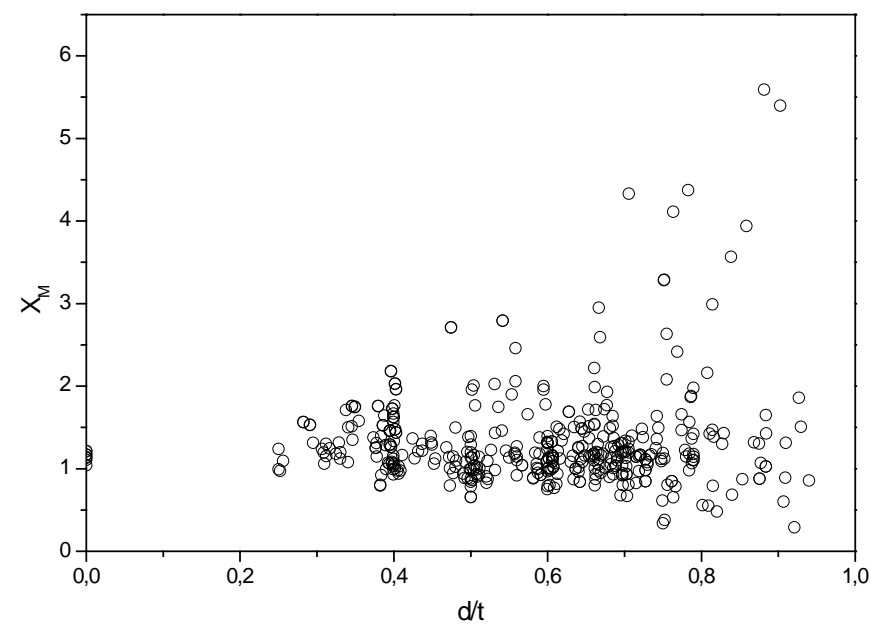

Figura 6.20. Comportamento da variável erro de modelo versus a razão $d / t$.
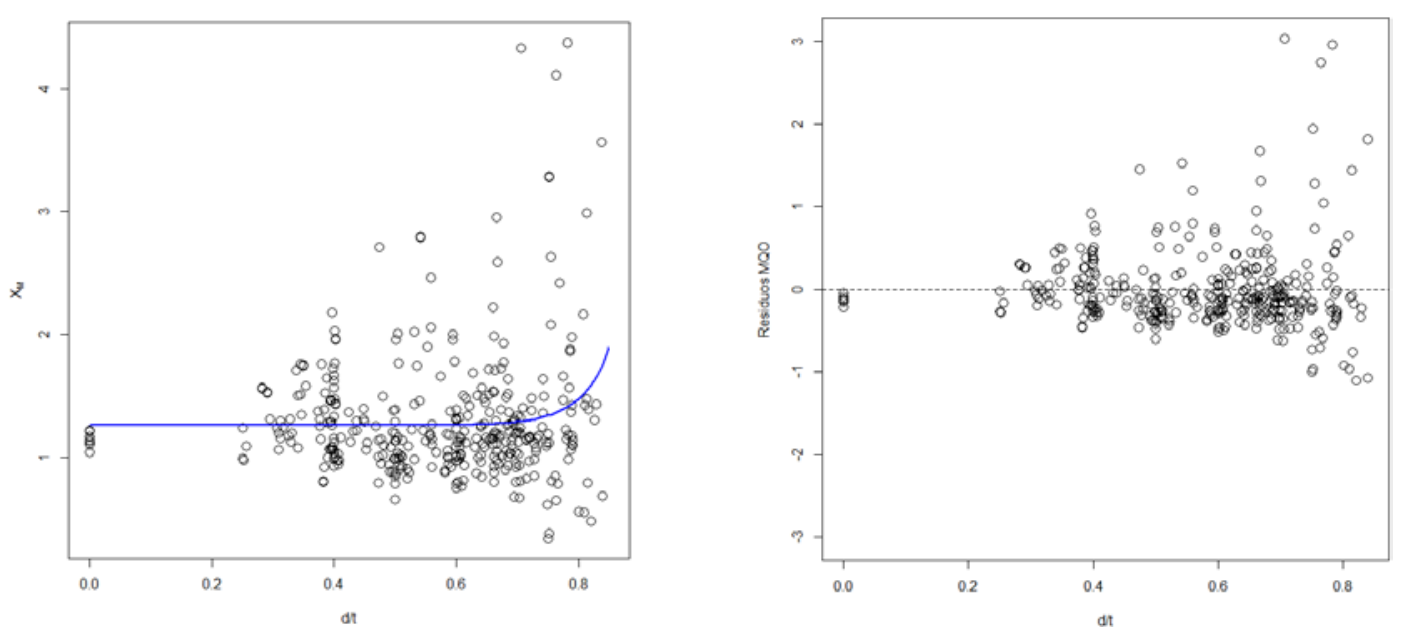

Figura 6.21.(a) Regressão não linear das variáveis $X_{M}$ versus razão $d / t$. (b) Variância do erro proporcional a $d / t$. 
A partir da função de ajuste é possível obter um desvio padrão de $\sigma=0.5008$, o que indica que os dados não estão próximos da média, conforme Figura 6.21b. A partir da Figura 6.21b é possível afirmar que não se satisfaz o suposto de homoscedasticidade. Na Tabela 6.12 apresentam-se os coeficientes obtidos por meio do ajuste pelo método dos MQO.

Após obter o modelo de regressão não-linear, ficam em evidência alguns problemas estatísticos envolvidos nos dados. Na Figura 6.21b observa-se que não existe variância uniforme nos resíduos. A não uniformidade pode existir pela presença de heteroscedasticidade. A existência pode ser confirmada a partir do método gráfico ou outro teste aplicado (Figura 6.22).

Tabela 6.12. Coeficientes ajustados da regressão não linear, modelo ASME B31G.

\begin{tabular}{ccc}
\hline Coeficientes & MQO & MQP \\
\hline$a$ & 0,79417 & 0,79120 \\
$b$ & $-2,48987$ & $-4,49474$ \\
$c$ & 13,75591 & 16,3964 \\
\hline
\end{tabular}

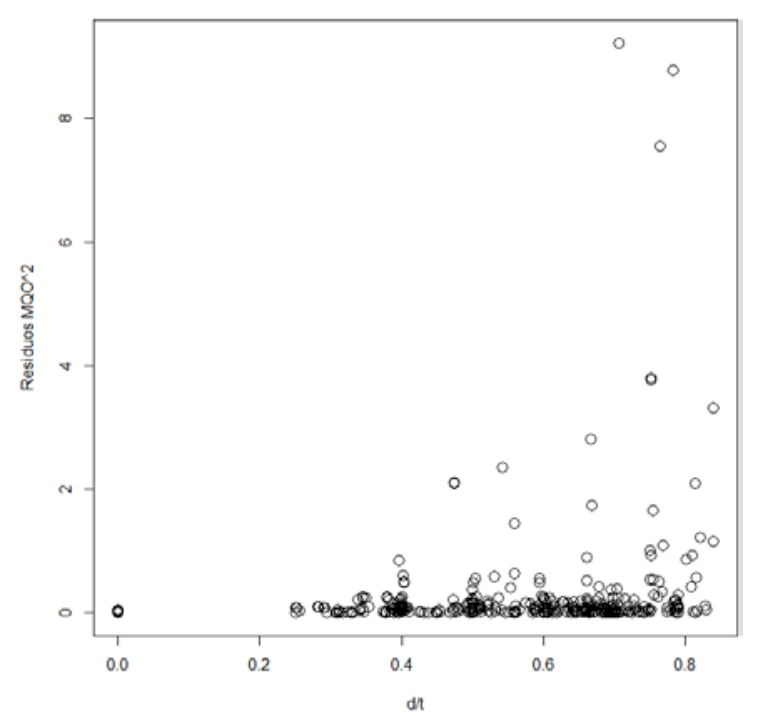

Figura 6.22. Resíduos estimados pelo MQO ao quadrado.

A Figura 6.22 apresenta um padrão sistemático definido. O comportamento dos dados pode ser comparado com os padrões sistemáticos apresentados na Figura 4.5. Por meio destes 
padrões é possível confirmar a presença de heteroscedasticidade. Para a solução deste tipo de problema, é aplicado o método dos Mínimos Quadrados Ponderados (MQP).

A aplicação do método MQP requer uma análise de regressão, a partir do valor absoluto dos resíduos estimados pelo método MQO, para assim, obter uma função que descreva o comportamento do desvio padrão. A análise de regressão é realizada a partir do valor absoluto dos resíduos estimados e a razão $d / t$, conforme Figura 6.23. A função de ajuste apresenta o seguinte comportamento:

$$
\hat{s}=\frac{1}{3.7988-8.7823 \cdot\left(\frac{d}{t}\right)^{6.3111}},
$$

sendo $\hat{s}$ o modelo de regressão estimado do desvio padrão. Esta função de ajuste é representada graficamente conforme Figura 6.23.

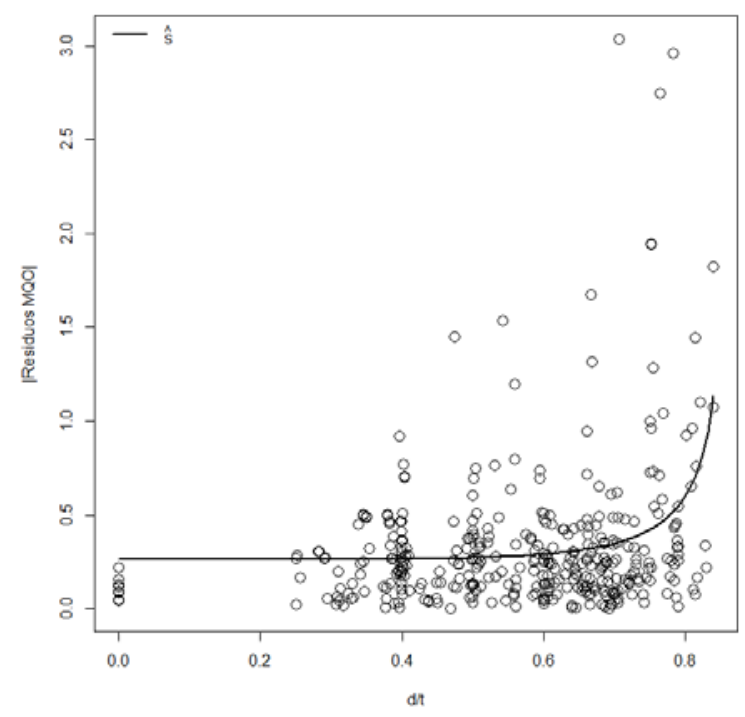

Figura 6.23. Regressão não-linear do valor absoluto dos resíduos estimados pelo método de ajuste MQO.

Na sequência, depois de realizada a análise de regressão do desvio padrão, obtém-se os pesos. Os pesos (Figura 6.24) são obtidos por meio da Equação (4.51), os quais são utilizados para a ponderação de cada observação. Por fim, proporcionando os pesos ao método MQP, é possível realizar uma análise de regressão, para assim, obter uma melhor representação do comportamento dos dados. A função ajustada tem um comportamento não-linear (Equação 
(6.4)), e seus coeficientes foram minimizados pelo método MQP (Tabela 6.12). A função de ajuste é plotada na Figura 6.25a, apresentando um valor do desvio padrão grande $(\sigma=1.435)$. Isto indica que os dados estão mais espalhados em comparação ao método MQO $(\sigma=0.5008)$ , embora apresente pouca heteroscedasticidade, a maioria dos dados são representados pelo modelo de regressão adotado (Figura 6.25b).

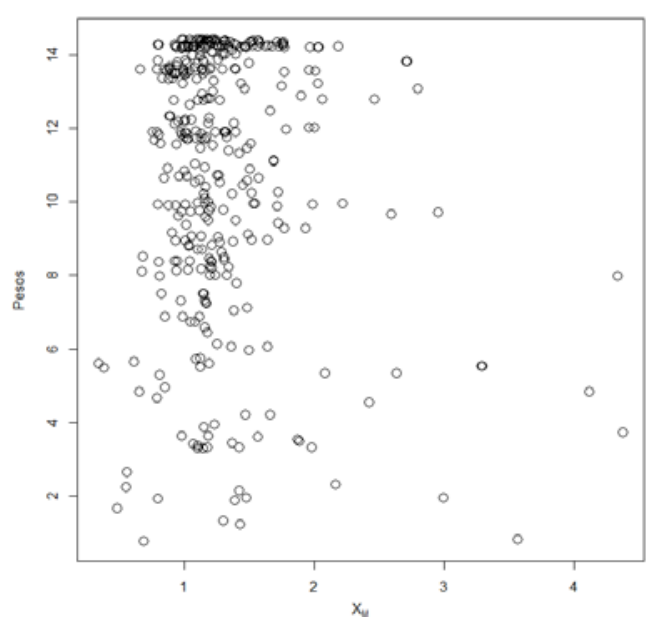

Figura 6.24. Pesos obtidos para cada observação.

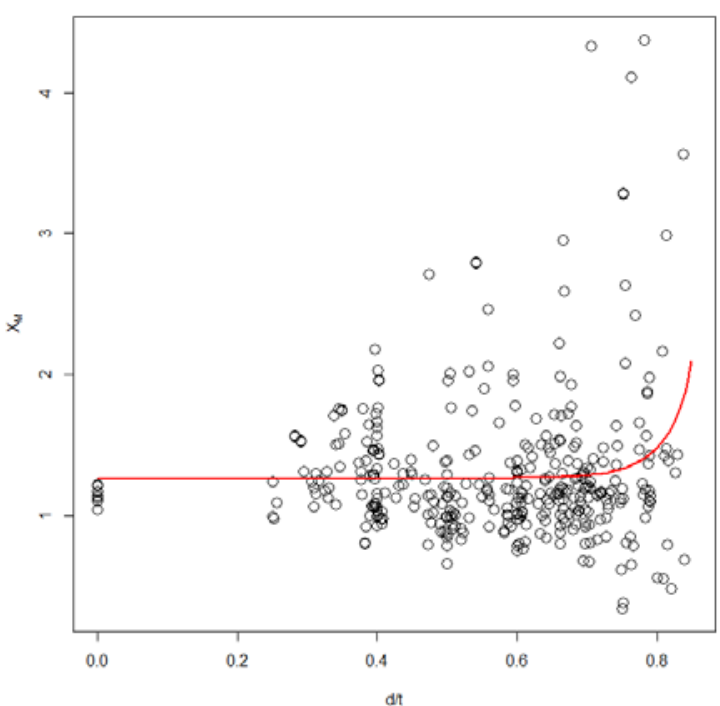

(a)

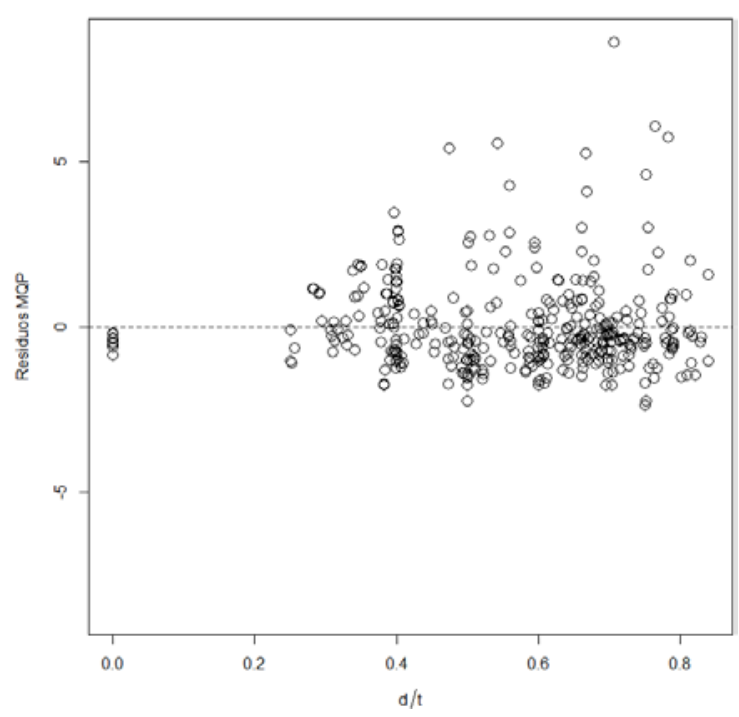

(b)

Figura 6.25. (a) Regressão não linear das variáveis $X_{M}$ versus razão $d / t$.

(b) Variância do erro uniforme proporcional à razão $d / t$.

Na Figura 6.25b, os resíduos estimados pelo método MQP apresentam uma tendência a se encontrar distribuídos aleatoriamente em torno de zero, mas ainda com algumas variações 
fora da faixa homogênea. Estes dados fora da faixa de homogeneidade não foram representados pelo modelo de regressão utilizado. Assim, é possível concluir que os resultados dos resíduos estimados ajustados pelo método MQP satisfazem o suposto de homoscedasticidade do modelo não-linear.

Na Tabela 6.12 tem-se os coeficientes do modelo de regressão obtidos pelos métodos MQO e MQP, os quais apresentam uma diferença mínima, sendo suficiente para garantir uma variância homogênea. De maneira geral, ambos os métodos de ajuste possuem o mesmo modelo de regressão, apesar de ser destacado que o método MQO apresenta heteroscedasticidade. Isto torna o método MQP adequado para minimizar os erros, e assim, obter uma modelo de regressão que represente o problema físico. Cabe lembrar que estes modelos não podem ser comparados, já que as variáveis não são as mesmas depois de aplicado os pesos.

\subsubsection{Modelo ASME B31G modificado}

Utilizando a mesma quantidade de dados do modelo anterior, o modelo ASME B31G modificado conta com 425 dados de defeitos reais e artificiais (Tabela 6.2). Estes dados são plotados e seu comportamento é mostrado na Figura 6.26.

Na Figura 6.26 encontram-se todas as observações disponíveis para as análises. Para uma melhor utilização, deve-se realizar uma filtragem nos dados conforme foi realizado no modelo

$\operatorname{DNV}(d / t \leq 0,85)$. Ressaltasse que para defeitos de corrosão com perda de espessura da parede entre $20 \%$ e $80 \%$ o duto deve ser reparado ou substituído segundo a ASME B31G. Para esta limitação o volumem de dados é muito baixo, não contaminando os resultados obtidos. Assim, pode-se obter uma nova base refinada de 405 dados com defeitos de corrosão real e artificial (Tabela 6.3).

Para obter a máxima informação dos dados, deve-se encontrar um modelo de regressão que represente seu comportamento. Desta maneira, uma análise de regressão não-linear é realizada e os resultados encontram-se plotados no gráfico da Figura 6.27a. A Figura 6.27b apresentam os resíduos do modelo. 


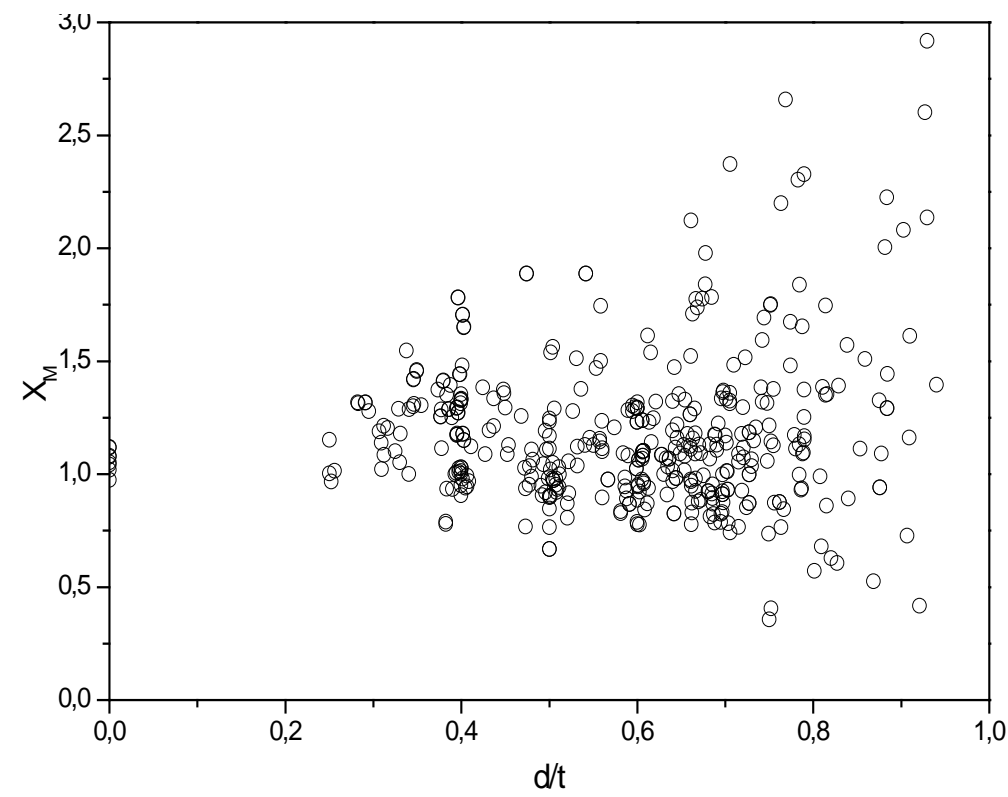

Figura 6.26. Comportamento da variável erro de modelo $X_{M}$ versus razão $d / t$, ASME B31G modificado.
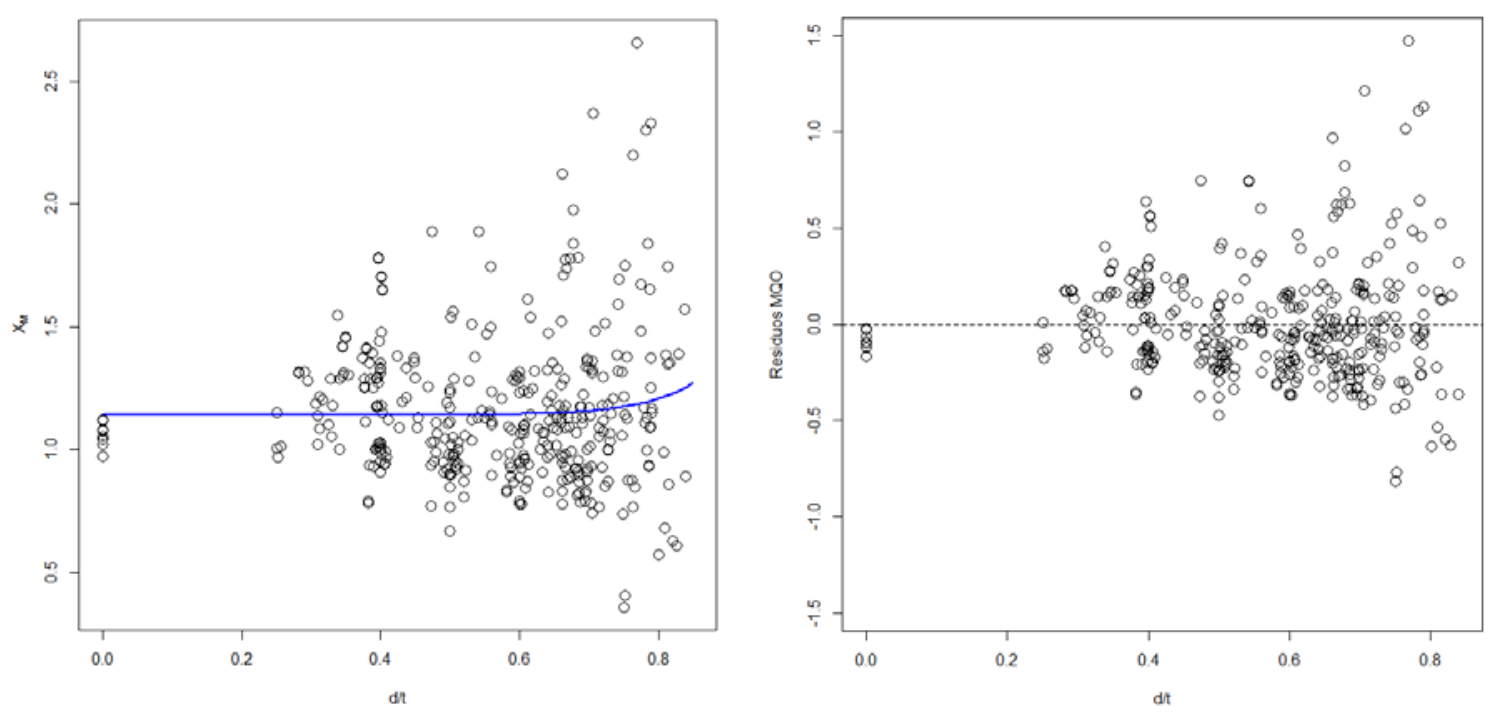

Figura 6.27.(a) Regressão não linear das variáveis $X_{M}$ versus razão $d / t$.

(b) Variância do erro proporcional à razão $d / t$.

De acordo com a Figura 6.27a, pode-se confirmar que a análise de regressão não-linear ajustada pelo método MQO, não representa o comportamento dos dados. Isto acontece pela grande dispersão dos dados em relação à linha de ajuste. Este análise de regressão apresentou um desvio padrão pequeno $(\sigma=0,2957)$. Mas observa-se que na Figura 6.27b, apesar do desvio padrão ser pequeno, não indica que os dados estejam próximos da média. Isto deve ser 
estudado com mais detalhe já que é um problema estatístico. Os coeficientes obtidos para o modelo de regressão não-linear (Equação (6.4)) são apresentados na Tabela 6.13.

Após, realizado a análise de regressão é possível observar que os dados apresentam um problema estatístico, pois na Figura 6.27b os erros não possuem variância uniforme. Para contornar tal problema, aplicam-se os conhecimentos dos modelos anteriores, para verificar se há presença de heteroscedasticidade. O método gráfico é aplicado, conforme Figura 6.28.

Tabela 6.13. Coeficientes ajustados de regressão, método B31G modificado.

\begin{tabular}{ccc}
\hline Coeficientes & MQO & MQP \\
\hline$a$ & 0,87607 & 0,87184 \\
$b$ & $-0,48343$ & $-1,17987$ \\
$c$ & 10,40341 & 14,34672 \\
\hline
\end{tabular}

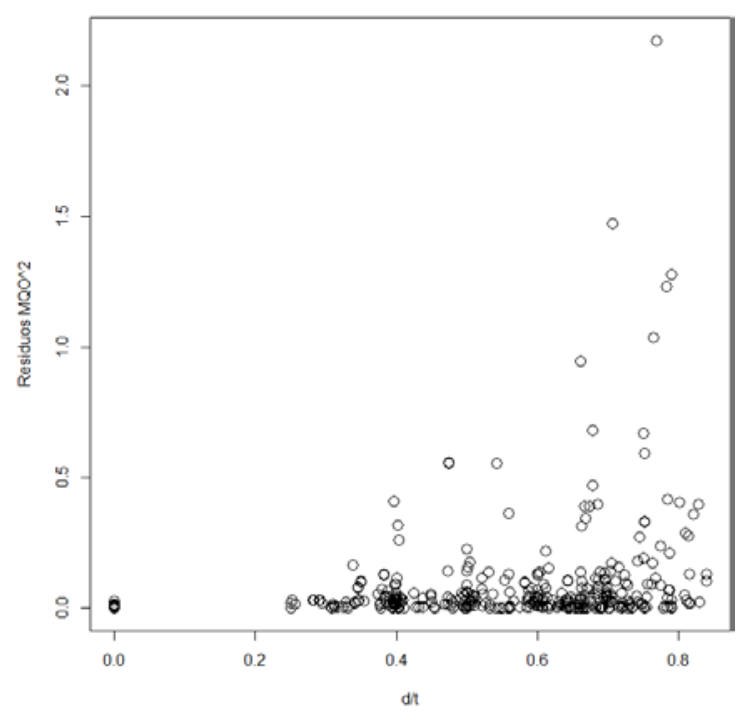

Figura 6.28. Valor quadrático dos resíduos estimados pelo método dos MQO.

Na sequência, tem-se na Figura 6.28 um comportamento crescente dos resíduos. Este tipo de comportamento é comparado com os padrões sistemáticos estabelecidos na Figura 4.5. Assim, confirma-se a existência de heteroscedasticidade. Este problema pode ser solucionado aplicando o método MQP. Para a aplicação do método, primeiro deve-se obter o valor absoluto dos resíduos estimados pelo método MQO, para depois realizar uma análise de regressão e encontrar um modelo que represente o comportamento do desvio padrão, conforme Figura 6.29. 


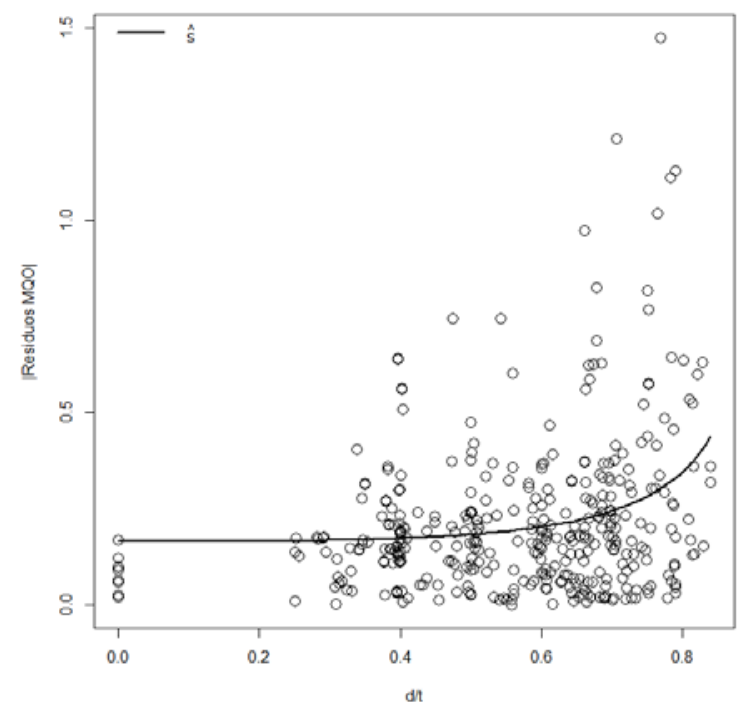

Figura 6.29. Valor absoluto dos resíduos estimados pelo método MQO.

A partir do gráfico da Figura 6.29, foi possível ajustar um modelo de regressão não-linear e assim representar o comportamento do desvio padrão que descreve uma relação entre $\sigma_{i}$ e razão $d / t$. Este modelo é dado por:

$$
\hat{s}=\frac{1}{6,0505-7,02 \cdot\left(\frac{d}{t}\right)^{3,5555}},
$$

onde $\hat{s}$ é a função de ajuste que descreve ao desvio padrão. Com a função estimada do desvio padrão, obtém-se os pesos que são utilizados para ponderar cada observação, conforme Equação (4.51). A ponderação dos pesos depende da variância de cada observação, já que os pesos são inversamente proporcionais à variância. Portanto, para cada observação são obtidos os pesos e em seguida eles são plotados, conforme ilustra a Figura 6.30 .

Após obtidos os pesos, é possível aplicar o método MQP. Para obter uma representação adequada dos dados, realiza-se uma análise de regressão não-linear e estimam-se os coeficientes que possuem variância mínima (Tabela 6.13). Na Figura 6.31a encontra-se uma função ajustada que apresenta um desvio padrão grande $(\sigma=1,31)$. Observa-se que os resíduos estão mais espalhados em comparação ao método MQO $(\sigma=0,2957)$, não apresentando problemas de heteroscedasticidade (Figura 6.31b). 


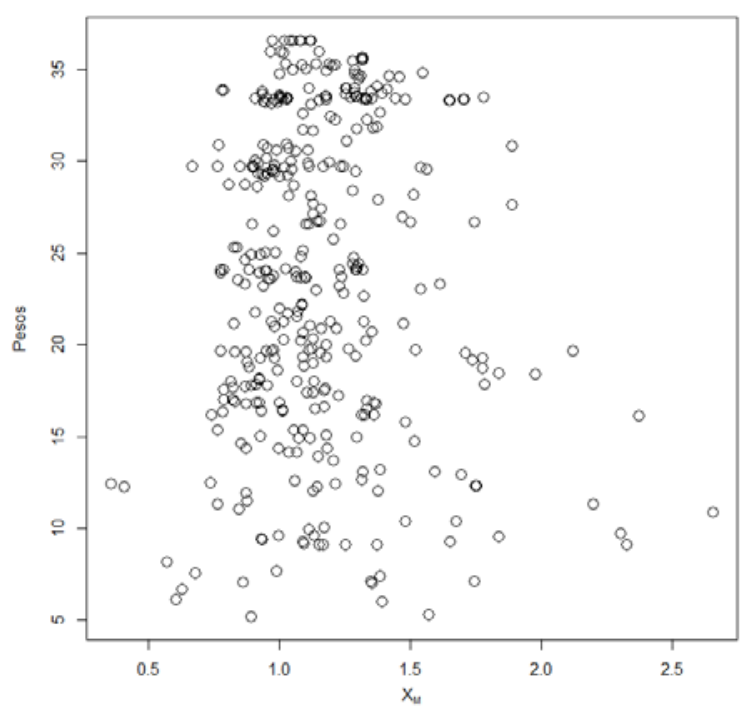

Figura 6.30. Pesos obtidos para cada observação.

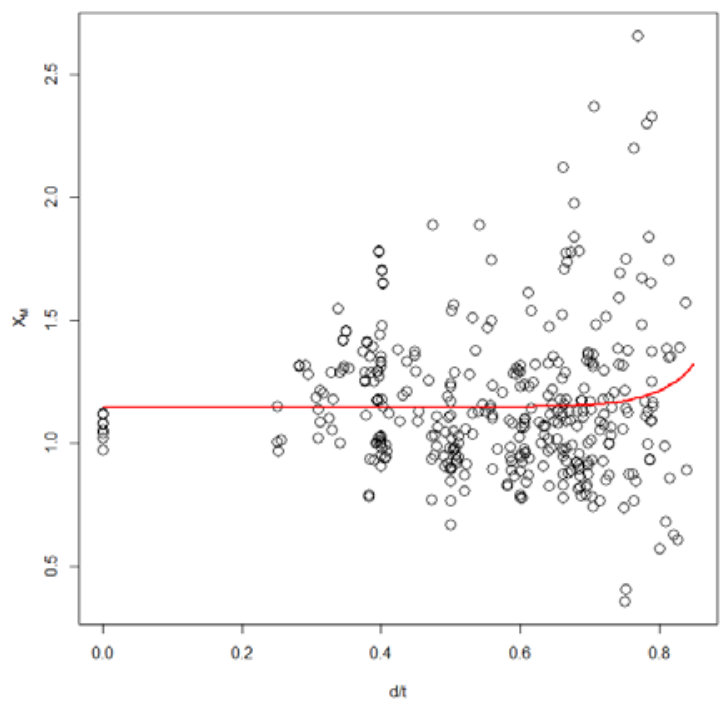

(a)

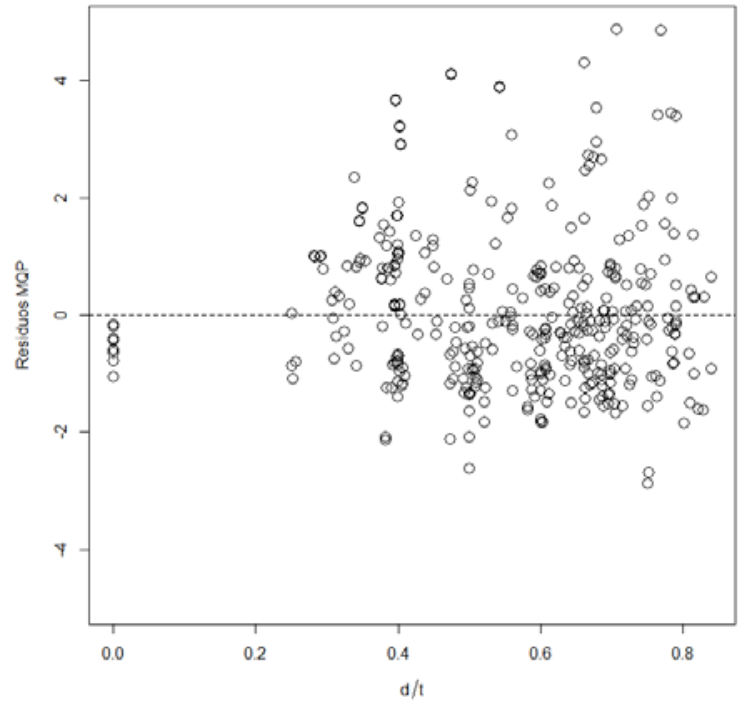

(b)

Figura 6.31. (a) Regressão não linear das variáveis $X_{M}$ vs. $d / t$

(b) Variância do erro uniforme proporcional a $d / t$.

Na Figura 6.31b os resíduos estimados pelo método dos MQP encontram-se distribuídos aleatoriamente em torno de zero, com um comportamento menos tendencioso que o obtido pelo método MQO tendência. A partir disto, é possível concluir que os resíduos satisfazem o suposto de homoscedasticidade do modelo não linear. 
Nas Figura 6.27b e Figura 6.31b, é possível visualizar a diferença entre os comportamentos dos resíduos dos modelos não lineares obtidos pelos métodos MQO e MQP. De maneira geral, ambos os métodos apresentam comportamentos similares na função de ajuste, mas as diferenças são suficientes para garantir homoscedasticidade. Ressalta-se que o método MQO apresenta heteroscedasticidade. Isto torna o método MQP mais adequado para representar o problema físico de dutos corroídos. Estes métodos não podem ser comparados, já que as variáveis são diferentes depois de serem aplicados os pesos.

Com a solução deste tipo de problema, é possível ter maior confiança nos ajustes realizados para assim conseguir descrever os fenômenos físicos presentes nos dutos corroídos.

\subsection{Análise de confiabilidade}

Para verificar a contribuição da variável erro de modelo, em função da razão $d / t$, na confiabilidade dos dutos corroídos é realizada análise de confiabilidade em um exemplo de um duto. É considerado o exemplo de Bazán e Beck (2011) e o modelo semi-empírico DNV RP F101. A função de estado limite é:

$g=p_{\text {cap }}-p$,

onde $P_{\text {cap }}$ é a capacidade de pressão de ruptura e $p$ é a pressão interna do duto independente do tempo. A capacidade de pressão de ruptura é dada pelo modelo da DNV RP F101:

$P_{\text {cap }}=X_{M} \cdot \frac{2 \cdot t \cdot \sigma_{u}}{D-t} \cdot\left[\frac{1-\left(\frac{d}{t}\right)}{1-\left(\frac{d}{t}\right) \cdot M^{-1}}\right]$.

Assim, a função de estado limite é dada por:

$g=\left[\frac{1}{a+b \cdot(d / t)^{c}}+\varepsilon\right] \cdot\left(\frac{2 \cdot t \cdot \sigma_{u}}{D-t} \cdot\right)\left[\frac{1-\left(\frac{d}{t}\right)}{1-\left(\frac{d}{t}\right) \cdot M^{-1}}\right]-p$, 
sendo $\varepsilon$ a variável erro independente que apresenta uma distribuição $N \sim\left(0, \sigma_{i}^{2}\right)$. Para o modelo DNV RP F101, os parâmetros $a, b, c$ são apresentados na Tabela 6.10 e o desvio padrão $(\sigma)$ encontra-se na seção (6.4.4.1).

De acordo com Zhou (2010) em um defeito de corrosão ativo, um duto pode falhar por pequeno vazamento ou por ruptura. Um pequeno vazamento ocorre quando o defeito penetra a parede do tubo $(d>t)$; a ruptura ocorre quando a parede do tubo sofre colapso plástico devido à pressão interna na localização do defeito antes do defeito penetrar na parede do tubo.

Para comparar os valores de probabilidade de falha definidos com a aplicação do método FORM foi considerado o exemplo adotado por Bazan e Beck (2011). A Tabela 6.14 mostra as distribuições estatísticas utilizadas para as variáveis aleatórias do estado limite de ruptura. As distribuições e parâmetros de pressão interna $(p)$, profundidade do defeito $(d)$, espessura da parede $(t)$, resistência à tração última $\left(\sigma_{u}\right)$, comprimento de defeito $(L)$ e fator de incerteza de modelo $\left(X_{M}\right)$, foram seguidas as recomendações da DNV RP F101 (2004). Ressalta-se que os valores estatísticos para o fator de incerteza do modelo $\left(X_{M}\right)$ foram cálculos pelo autor no presente trabalho.

A seguir serão apresentados os casos analisados via programa StRAnD. Será considerado um duto de aço X42, com as seguintes características:

1. Diâmetro nominal exterior do duto: 16 in (406.4 mm)

2. Espessura nominal da parede: $6.4 \mathrm{~mm}$

3. MAOP: $940 \mathrm{psi}(6.5 \mathrm{MPa})$

4. SMTS: $60,000 \mathrm{psi}(414 \mathrm{MPa})$

5. SMYS:42,000 psi (290 MPa)

Na Figura 6.32 mostra-se a variação da pressão de ruptura (burst) média em função do crescimento do defeito. 
Tabela 6.14. Caracterização das variáveis aleatórias do duto exemplo (Bazán e Beck, 2011).

\begin{tabular}{cccc}
\hline Variável & Distribuição & $\mu$ & Incerteza \\
\hline$D$ & Determinística & Valor nominal & --- \\
$p$ & Gumbel & $1,05 M A O P$ & $C O V=3 \%$ \\
$t$ & Normal & $t$ & $C O V=3 \%$ \\
$\sigma_{u}$ & Normal & $1,09 S M T S$ & $C O V=3 \%$ \\
$L$ & Normal & $L$ & $\sigma=19,5 \mathrm{~mm}$ \\
$d$ & Lognormal & $d$ & $C O V=50 \%$ \\
$\varepsilon$ & Normal & 0,00 & $\sigma=1,259$ \\
\hline
\end{tabular}

$C O V=$ coeficiente de variação; $\sigma=$ desvio padrão; $t=$ espessura da parede; SMTS = resistência a tração mínima especificada; $L=$ comprimento do defeito; $d=$ profundidade do defeito.

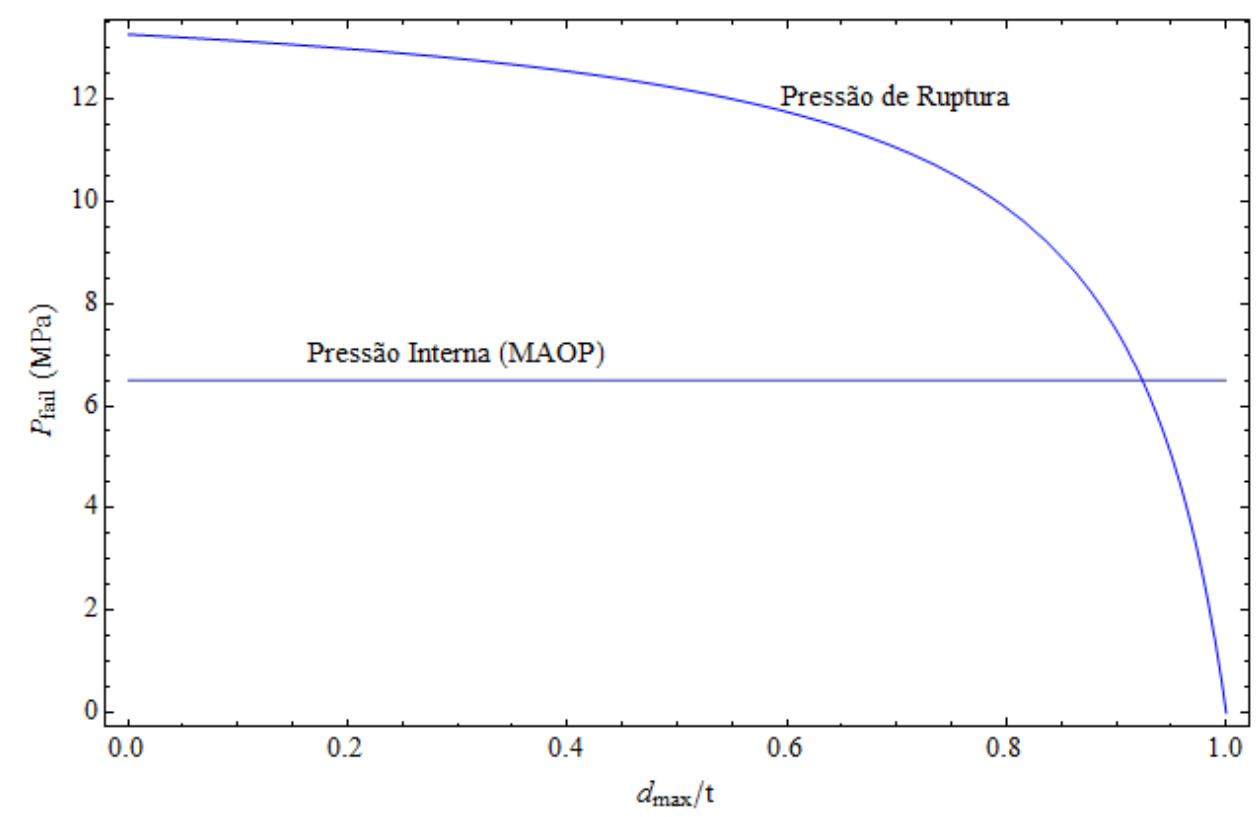

Figura 6.32. Pressão de ruptura média em função da razão $d / t$.

A Figura 6.32 ilustra a pressão de operação (MAOP) e a evolução temporal da pressão de ruptura do duto em função da razão $d / t$. Observa-se que a vida do duto varia significativamente em função da razão $d / t$. Desta forma, uma mesma pressão interna aplicada pode levar a falha do duto ou não, a depender da razão $d / t$. 
A probabilidade de falha $\left(P_{f}\right)$ foi calculada pelo método FORM (First Order Reliability Method) para diferentes profundidades do defeito. Na Tabela 6.15 encontra-se a probabilidade de falha elevada à potência dez, que indica a ordem da magnitude. A Tabela 6.15 apresenta os índices de confiabilidade e os coeficientes de sensibilidade das probabilidades de falha calculadas para o duto de referência (X42, $D=406,4 \mathrm{~mm}, P=6,05 \mathrm{MPa}$ ). Os coeficientes de sensibilidade mostram a contribuição de cada variável aleatória à probabilidade de falha resultante. Na Figura 6.33 observam-se como os coeficientes de sensibilidade mudam à medida que o defeito avança em profundidade (razão $d / t$ ). A Figura 6.33 mostra que à medida que o defeito cresce e se torna mais próximo da espessura da parede, a importância de $\left(X_{M}\right)$ diminui, passando a ter maior contribuição à incerteza na profundidade do defeito. Também pode ser visto que para quaisquer valores da razão $d / t$ a variável que tem maior contribuição para a probabilidade de falha é o erro de modelo $\left(X_{M}\right)$. Esta variável representa os erros envolvidos na previsão da pressão de ruptura do duto.

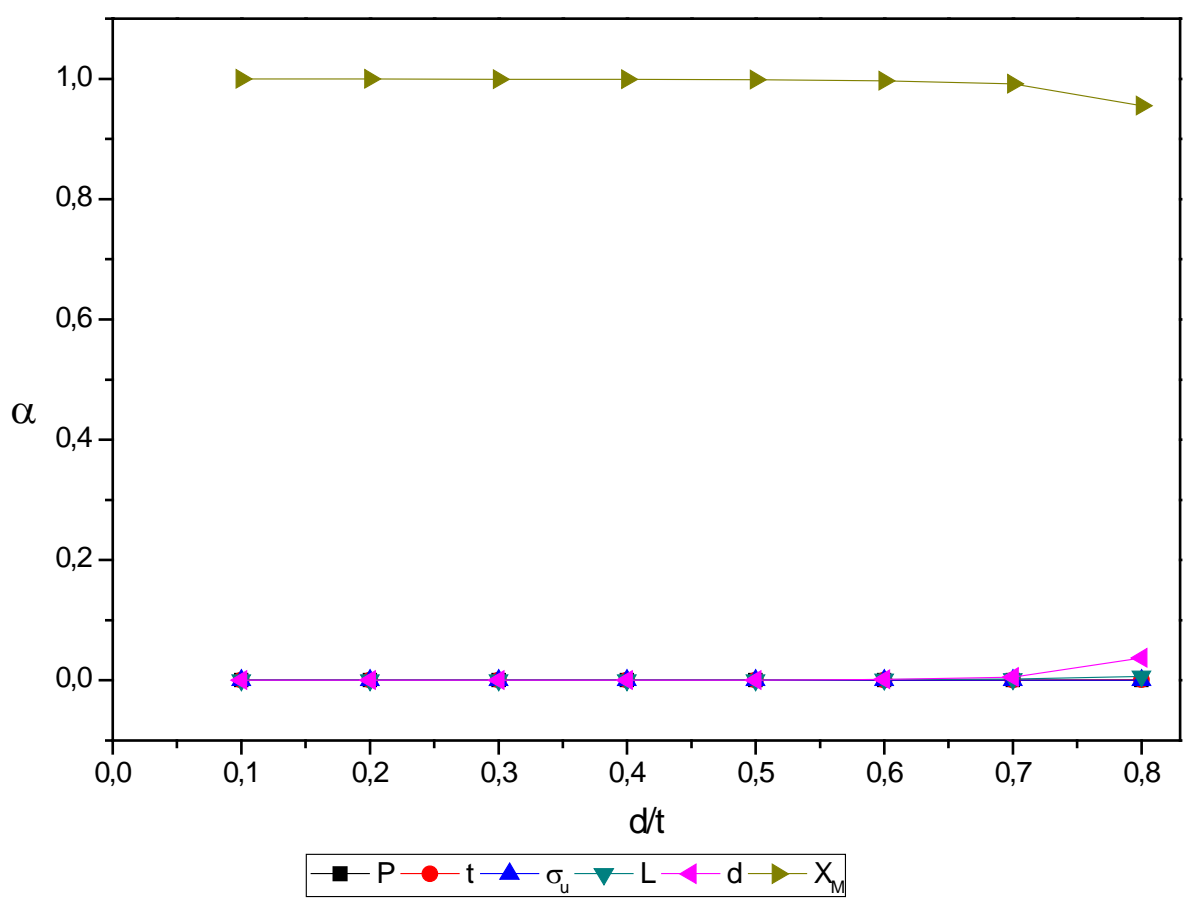

Figura 6.33. Coeficientes de sensibilidade para um duto de aço X42. 


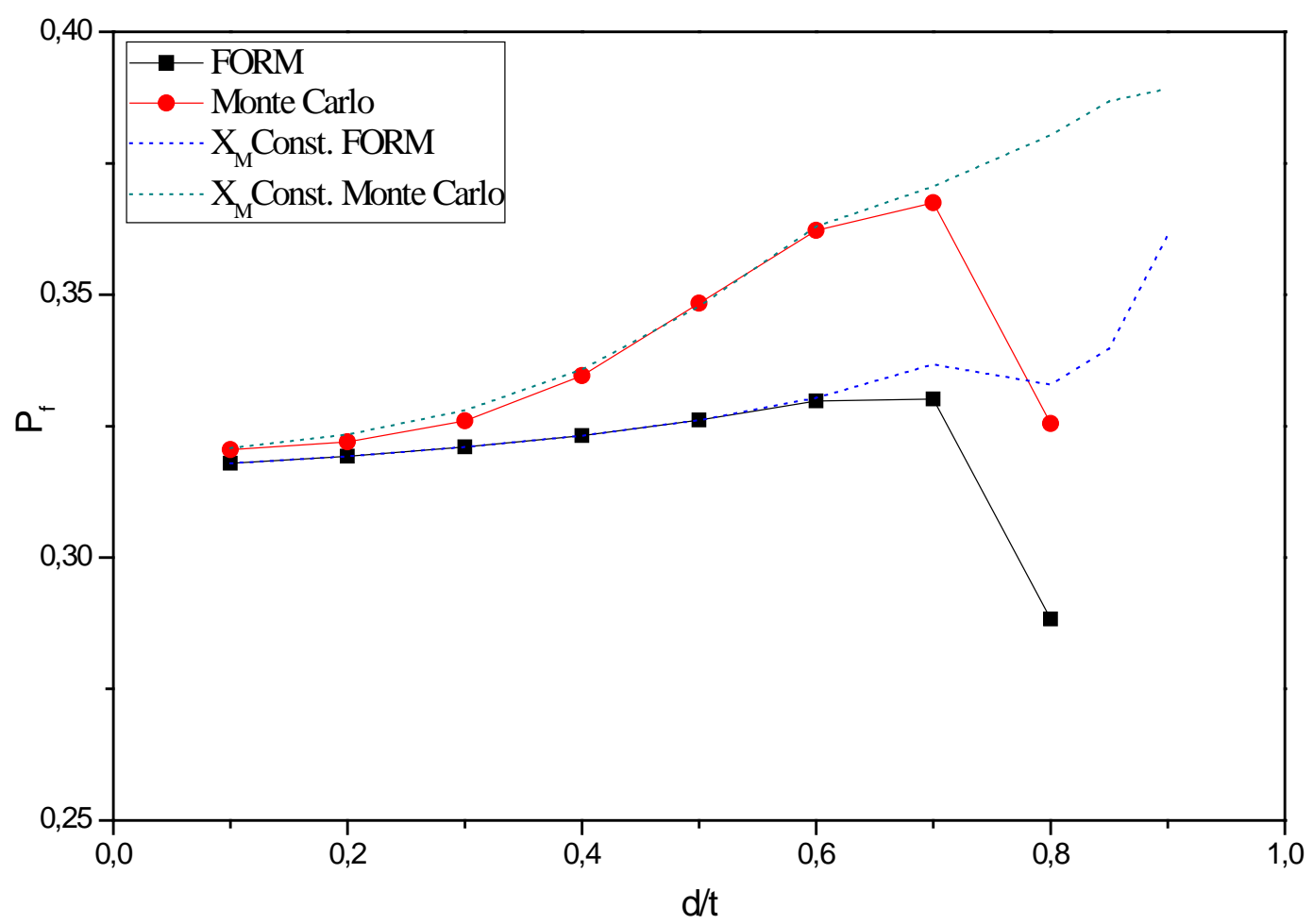

Figura 6.34. Probabilidade de falha versus razão $d / t$.

A Figura 6.34 mostra as probabilidades de falha em função da razão d/t, para um duto de aço X42 que apresenta defeito de corrosão. Na Figura 6.34 comparam-se os resultados obtidos via FORM (quadrados) com os resultados obtidos via simulação de Monte Carlo (pontos), utilizando $10^{3}$ amostras. Em geral observa-se que as probabilidades de falha aumentam com a razão $d / t$ entre 0,1 e 0,7 , conforme esperado. Para valores da razão $d / t>0,7$ observa-se que a probabilidade de falha diminui, o que vai contra a intuição. Isto acontece por que o erro de modelo médio se torna cada vez mais conservador. Portanto, para a razão $d / t>0,7$, o efeito do aumento da média de $X_{M}$ é maior do que o efeito do aumento da profundidade relativa do defeito $(d / t)$. Para confirmar esta observação, a linha pontilhada cinza representa a probabilidade de falha obtida via Monte Carlo, assumindo um valor médio constante da variável erro de modelo, observando-se que para valores constantes da variável erro de modelo a probabilidade de falha tem um comportamento sempre crescente com a razão d/t. Isto também ocorre para a linha pontilhada azul obtida via FORM, assumindo um valor médio constante da variável erro de modelo. No entanto para razão d/t entre 0,7 e 0,8 a probabilidade de falha diminui devido à variação do ponto de projeto, como pode ser visto na Tabela 6.15. 
A variação que se apresenta no método FORM se deve à não linearidade da equação de estado limite adotada. Conclui-se que a função de ajuste para obter o valor médio da variável erro de modelo determinada neste trabalho, para o modelo DNV, só é valida para valores da razão $d / t$ menores que 0,7 .

Destaca-se que as análises realizadas por Bazán e Beck (2011) apresentaram uma probabilidade de falha em torno de 0,134 , sendo semelhantes aos resultados obtidos neste trabalho.

É importante ressaltar que estes resultados foram obtidos por meio do programa StRAnD Structural Reliability Analysis and Design, desenvolvido no Departamento de Engenharia de Estruturas da EESC/USP. 
Tabela 6.15. Resultados obtidos da análise de confiabilidade a partir do programa StRAnD.

\begin{tabular}{c|c|cccccccccc}
\hline & $\mathrm{d} / \mathrm{t}$ & 0,1 & 0,2 & 0,3 & 0,4 & 0,5 & 0,6 & 0,7 & 0,8 & 0,85 & 0,9 \\
& $\mathrm{D}$ & 0,64 & 1,28 & 1,92 & 2,56 & 3,2 & 3,84 & 4,48 & 5,12 & 5,44 & 5,76 \\
\hline$\alpha$ & $D$ & $2,71 \mathrm{E}-06$ & $1,65 \mathrm{E}-05$ & $5,92 \mathrm{E}-05$ & 0,00018 & 0,00052 & 0,00157 & 0,00549 & 0,03709 & 0,98956 & 0,99599 \\
& $X_{M}$ & 0,999606 & 0,99955 & 0,99942 & 0,99915 & 0,99851 & 0,99685 & 0,99157 & 0,95496 & 0,00325 & $4,93 \mathrm{E}-08$ \\
\hline \multirow{2}{*}{ FORM } & $\beta$ & 0,473539 & 0,46969 & 0,46488 & 0,45871 & 0,45058 & 0,44055 & 0,43948 & 0,55832 & 0,51784 & $-0,4622$ \\
& $P_{f}$ & $3,18 \mathrm{E}-01$ & $3,19 \mathrm{E}-01$ & $3,21 \mathrm{E}-01$ & $3,23 \mathrm{E}-01$ & $3,26 \mathrm{E}-01$ & $3,30 \mathrm{E}-01$ & $3,30 \mathrm{E}-01$ & $2,88 \mathrm{E}-01$ & $3,02 \mathrm{E}-01$ & $6,78 \mathrm{E}-01$ \\
\hline \multirow{2}{*}{ Monte Carlo } & $\beta$ & $4,66 \mathrm{E}-01$ & 0,46211 & 0,45099 & 0,42725 & 0,38964 & 0,35258 & 0,33848 & 0,45373 & $7,95 \mathrm{E}-01$ & $-1,49 \mathrm{E}+00$ \\
& $P_{f}$ & $3,21 \mathrm{E}-01$ & $3,22 \mathrm{E}-01$ & $3,26 \mathrm{E}-01$ & $3,35 \mathrm{E}-01$ & $3,48 \mathrm{E}-01$ & $3,62 \mathrm{E}-01$ & $3,68 \mathrm{E}-01$ & $3,26 \mathrm{E}-01$ & $2,13 \mathrm{E}-01$ & $9,32 \mathrm{E}-01$ \\
\hline \multirow{3}{*}{ XM const. FORM } & $\beta$ & $4,74 \mathrm{E}-01$ & $4,70 \mathrm{E}-01$ & $4,65 \mathrm{E}-01$ & 0,45871 & 0,45049 & 0,43894 & 0,4214 & 0,43185 & $4,13 \mathrm{E}-01$ & $3,55 \mathrm{E}-01$ \\
& $P_{f}$ & $3,18 \mathrm{E}-01$ & $3,19 \mathrm{E}-01$ & $3,21 \mathrm{E}-01$ & $3,23 \mathrm{E}-01$ & $3,26 \mathrm{E}-01$ & $3,30 \mathrm{E}-01$ & $3,37 \mathrm{E}-01$ & $3,33 \mathrm{E}-01$ & $3,40 \mathrm{E}-01$ & $3,61 \mathrm{E}-01$ \\
\hline \multirow{2}{*}{ XM const. Monte Carlo } & $\beta$ & $4,65 \mathrm{E}-01$ & 0,458212 & 0,445443 & 0,42395 & 0,39127 & 0,35045 & 0,33027 & 0,30443 & $2,88 \mathrm{E}-01$ & $2,81 \mathrm{E}-01$ \\
& $P_{f}$ & $3,21 \mathrm{E}-01$ & $3,23 \mathrm{E}-01$ & $3,28 \mathrm{E}-01$ & $3,36 \mathrm{E}-01$ & $3,48 \mathrm{E}-01$ & $3,63 \mathrm{E}-01$ & $3,71 \mathrm{E}-01$ & $3,80 \mathrm{E}-01$ & $3,87 \mathrm{E}-01$ & $3,89 \mathrm{E}-01$ \\
\hline
\end{tabular}




\section{CONCLUSÕES}

Neste trabalho foi apresentado um estudo de modelos empíricos de previsão da pressão de ruptura de dutos contendo defeitos de corrosão. Além disso, foi feita uma análise de confiabilidade e realizou-se uma análise do fator de importância para cada variável.

Demostrou-se que os resultados dos ensaios obtidos para defeitos reais e artificiais não podem ser considerados como sendo da mesma população, para os modelos DNV RP F101 e PCORRC. Em contrapartida, para os modelos ASME B31G e B31G modificado, os dados podem ser considerados da mesma população e ser combinados nas análises.

Os resultados das analises estatísticas e dos ajustes de distribuição para a variável erro de modelo foram comparados com dados na literatura (Zhou; Huang, 2012); tendo sido observadas importantes semelhanças. Os coeficientes de variação obtidos encontram-se na mesma faixa (em torno de 30\%) que os encontrados na literatura. Nestes resultados destaca-se que o modelo DNV RP F101 é considerado como o melhor modelo em relação àqueles estudados. A variável erro de modelo apresenta um valor médio menos conservador $(1,0937)$ sendo próximo da unidade, e um coeficiente de variação de (31,9\%), pequeno em comparação com os outros modelos de pressão de ruptura.

Para os diferentes modelos de predição da pressão de ruptura obteve-se um limite para a classificação dos dados, sendo que para os valores $\sigma_{y}>375 \mathrm{MPa}$ e $\sigma_{u}>475 \mathrm{MPa}$ (tensão de escoamento e tensão à tração última) a variável erro de modelo tende a ser menos conservadora, apresentando valores estatísticos menores. Em contrapartida, para tensão de escoamento e tensão à tração última maiores $\left(\sigma_{y} \leq 375 \mathrm{MPa}\right.$ e $\left.\sigma_{u} \leq 475 \mathrm{MPa}\right)$ a variável erro de modelo tende a ser mais conservadora, apresentando valores estatísticos maiores. De maneira geral, a partir destas classificações obteve-se que os modelos DNV RP F101 e o PCORRC são menos conservador entre aqueles utilizados, 
já que para tensões à tração última $\sigma_{u} \leq 475 \mathrm{MPa}$ e $\sigma_{u}>475 \mathrm{MPa}$, os valores estatísticos foram pequenos, próximos da unidade, ao se comparar com os outros modelos utilizados.

Os modelos de regressão não linear para cada modelo foram ajustados pelo método dos mínimos quadrados ponderados, para assim garantir a uniformidade da variância (homoscedasticidade). A variância obtida em todos os modelos de regressão para a variável erro de modelo versus a razão $d / t$ é em torno de $\sigma^{2}=1,3$. Conclui-se que há aleatoriedade em torno de zero, com um comportamento menos tendencioso que o obtido pelo método MQO (tendência à homoscedasticidade). Esta propriedade gera uma maior confiança nos modelos de regressão utilizados para a representação dos dados.

Foi realizada uma análise para avaliar a importância de cada uma das variáveis envolvidas no problema. Pôde-se concluir que variáveis aleatórias que apresentam coeficientes de sensibilidade pequenos podem ser tratadas como determinísticas simplificando o problema. Portanto, observou-se que a variável erro de modelo é a variável aleatória com maior contribuição à probabilidade de falha em dutos corroídos, já que para valores usuais da razão $d / t$ o coeficiente de sensibilidade $\left(\alpha^{2}\right)$ está em torno de 0,99 .

$\mathrm{Na}$ análise de confiabilidade realizada foi verificado que para valores da razão $d / t$ maiores que 0,7 a probabilidade de falha diminui. Isto acontece por que o erro de modelo médio se torna cada vez mais conservador. Portanto o modelo de regressão só deve ser utilizado para valores da razão $d / t$ menores que 0,7 . 
Ahammed, M. Probabilistic estimation of remaining life of a pipeline in the presence of active corrosion defects, International Journal of Pressure Vessels and Piping, v. 75, p.321-329, 1998.

Ang, A.H-S.; Tang, W.H. Probability Concepts in Engineering: Emphasis on Applications to Civil and Environmental Engineering. 2.ed. New York: John Wiley \& Sons, 2007.

API 5L, 2000, Specification for Line Pipe, American Petroleum Institute.

ASME B31.8, 1999, Gas Transmission and Distribution Piping Systems. New York, NY, American Society of Mechanical Engineers.

Bazán, F.A.V.; Beck, A.T. Reliability of Pipelines Under Corrosion and Mechanical Damage. In: 11th International Conference on Applications of Statistics and Probability in Civil Engineering (ICASP11), n. 275, Zurique, Switzerland, p. 2285-2292, 2011.

Beck, A.T. StRAnD: Manual do Usuário. São Carlos, SP, Brasil, EESC/USP, 2007.

Beck, A. T. Curso de confiabilidade estrutural - Notas de aula. São Carlos, SP, Brasil, Escola de Engenharia de São carlos - USP, 2012.

Benjamin, A.C.; Vieira, R.D.; Freire, J.L.F.; De Castro, J.T.P. Burst Tests on Pipeline with Long External Corrosion. Proceedings of the Third International Pipeline Conference (IPC 2000), Calgary, Alberta, Canada: American Society of Mechanical Engineers, v.2, p. 793-799, Out. 2000.

Besel, M.; Zimmermann, S.; Kalwa, C.; Köppe, T.; Liessem, A. Corrosion Assessment Method Validation for High-Grade Line Pipe. In: Proceedings of the 8th International Pipeline Conference (IPC 2010), Calgary, Alberta, Canada: American Society of Mechanical Engineers, v.4, p. 385-394, Out. 2000.

Bjørnøy O.H.; Sigurdsson, G.; Cramer, E.H. Residual strength of corroded pipelines, DNV test results. In: Tenth international conference on offshore and polar engineering (ISOPE 2000), Seattle, USA, 28 Maio - 2de Jun. 2000.

Cordeiro, A.L.R. Aplicação dos Metodos Form e Sorm para Avaliação de Dutos Corroídos. 2009. 96p. Dissertação (Mestrado em Mecanica) - PPGEM do centro de Ciências Exatas e de Tecnologia, Universidade Católica do Paraná, 2009.

Coulson, K.E.W.; Worthingham, R.G. Pipe Corrosion-1: Standard damage assessment approach is overly conservative, Oil \& Gas Journal, Abr. 1990. 
Coulson, K.E.W.; Worthingham, R.G. Pipe Corrosion-Conclusion: New guidelines promise more accurate damage assessment, Oil \& Gas Journal, Abr. 1990.

Chouchaoui, B.A.; Pick, R.J. Residual Burst Strength of Pipe with Internal Corrosion Pits. International Conference on Pipeline Reliability, Calgary, Canada, Jun. 1992.

Chouchaoui, B.A. Pic, R.J. Behaviour of Isolated Pits within General Corrosion. Pipes and Pipeline International, v.39, n.1, January, February, p. 12-21, 1994.

Chouchaoui, B.A.; Pick, R.J. Behaviour of Circumferentially Aligned Corrosion Pits. International Journal of Pressure Vessels and Piping, v. 57, p. 187-200, 1994.

Chouchaoui, B.A.; Pick, R.J. Behaviour of Longitudinally Aligned Corrosion Pits. International Journal of Pressure Vessels and Piping, v. 67, p. 17-35, 1996.

Cronin, D.S.; Pick, R.J. A New Multi-Level Assessment Procedure for Corroded Line Pipe. In: Proceedings of the Third. International Pipeline Conference (IPC 2000), Calgary, Alberta, Canada: American Society of Mechanical Engineers, v. 2, p. 801-808, Out. 2000.

Cronin, D.S.; Roberts, K.A.; Pick, R.J. Assessment of Long Corrosion Grooves in Line Pipe, Proceedings of the First International Pipeline Conference (IPC 1996), Calgary, Alberta, Canada: American Society of Mechanical Engineers, v. 1, 1996.

De Souza, R.D.; Benjamin, A.C.; Freire, J.L.F.; Vieira, R.D.; Diniz, J.L.C. Burst Tests on Pipeline Containing Long Real Corrosion Defects. International Pipeline Conference (IPC 2004), Calgary, Alberta, Canada: American Society of Mechanical Engineers, v.1-3, p. 11591167, Out. 2004.

Diniz, J.L.C.; Vieira, R.D.; Castro, J.T.; Benjamin, A.C.; Freire, J.L.F. Stress and Strain Analysis of Pipelines with Localized Metal Loss, Experimental Mechanics, v.46, n.6, p. 765775, Dez. 2006.

DNV-RP-F101. Recommended Practice: Corroded Pipelines. Høvik, Norway, Det Norske Veritas, 2004.

FU, B.; BATTE, A.D. Advanced Methods for the Assessment of Corrosion in Linepipe. Health and Safety Executive Summary Report, OTO 1999-051, HSE Books, 1999.

Gentil, V. Corrosão. LTC. 2011.

Gujarati, D.; Porter, D. Basic Econometrics. The McGraw-Hill/Irwin. 2009

Inkabi, K.S.; Bea, R.G. Burst Database Verification Study For Corroded Line-Pipe. In: $23^{\text {rd }}$. International Conference on Offshore Mechanics and Artic Engineering (OMAE 2004), Vancouver, British Columbia, Canada: American Society of Mechanical Engineers, v.2, p. 4553, Jun. 2004.

Kiefner, J.F.; Duffy, A.R. Criteria for Determining the Strength of Corroded Areas of Gas Transmission Lines. American Gas Association Operating Section on Transmission Conference, AGA, 1973.

Kiefner, J.F.; Marvin, C.W.; Duffy, A.R. Predicting the Strength of Corroded Line Pipe. World Gas Conference, IGU/C 42-73, 1973. 
Kiefner, J.F.; Maxey, W.A.; Eiber, R.J.; Duffy, A.R. Failure stress levels of flaws in pressurized cylinders. Progress in flaw growth and fracture toughness testing. ASTM STP536, American Society for Testing and Materials, p. 461-481, 1973.

Kiefner, J.F.; Vieth, P.H.; Roytman, I. Continued Validation of RSTRENG. Pipeline Research Committee of American Gas Association, 1995.

Kutner, M.H.; Nachtsheim, C.J.; Neter, J.; Li, W. Applied Linear Statistical Models. The McGraw-Hill/Irwin. 2005.

Leis, B.N.; Stephens, D.R. An alternative approach to assess the integrity of corroded line pipe part II: alternative criterion. In: Proceedings of the 7th International Offshore and Polar Engineering Conference, Honolulu, USA, p. 635-41, 25-30 de maio de 1997.

Mok, D.R.B.; Pick, R.J.; Glover, A.G. Behavior of Line Pipe with Long External Corrosion, Materials Performance, v. 29, n. 5, p. 75-79, Maio 1990.

Mok, D.R.B.; PICK, R.J.; Glover, A.G.; Hoff, R. Bursting of Line Pipe with Long External Corrosion, International Journal of Pressure Vessels and Piping, v. 46, p. 195-216, 1991.

Montgomery, D.C.; Runger, G.C. Applied Statistics and Probability for Engineers. John Wiley. 2003.

Nehoda, J.; Horalek. V. Long Term Experiences with the Maintenance of High Pressure Pipelines at Transgas s.p., In:Third International Conference on Pipeline Rehabilitation \& Maintenance, Prague, Czech Republic, p. 4-7, Set. 2000.

Penspen Group. The Pipeline Defect Assessment Manual (Joint Industry Project), 2002.

Roberts, K.A.; Pick, R.J. Correction for Longitudinal Stress in the Assessment of Corroded Line Pipe, In: Proceedings of Second International Pipeline Conference (IPC 1998), Calgary, Alberta, Canada: American Society of Mechanical Engineers, p. 553-561, Jun. 1998.

Sagrilo, L.V.S.; De Lima, E.C.P. Apostila de Confiabilidade Esturural. Metodos Analiticos FORM/SORM. Programa de Engenharia Civil, Coordenação dos Programas de Pós-Graduação em Engenharia. Universidade Federal do Rio de Janeiro, Rio de Janeiro, 2010.

Shannon, R.W.E. The failure behaviour of line pipe defects, International Journal of Pressure Vessels and Piping, v. 2, n.3, p. 243-255, 1974.

Stephens, D.R.; Bubenik, T.A.; Francini, R.B. Residual Strength of Pipeline Corrosion Defects Under Combined Pressure and Axial Loads, Final Report to Line Pipe Research Supervisory Committee of the Pipeline Research Committee of the American Gas Association, NG-18 Report No. 216, A.G.A. Catalog No. L51722, Battelle Memorial Institute, Fev. 1995.

Vieth, P.H.; Kiefner, J.F. Database of Corroded Pipe Test, In: Pipeline Research Committee of American Gas Association, 1994.

Zhou, W.; Huang, G.X. Model Error Assessments of Burst Capacity Models for Corroded Pipelines. International Journal of Pressure Vessels and Piping, v.99-100, p. 1-8, 20 de agosto de 2012.

Zhou, J.; Rothwell, B.; Nessim, M.; Zhou, W.; Reliability-Based Design and Assessment Standards for Onshore Natural Gas Transmission Pipelines, Journal of Pressure Vessel Technology, v. 131, n. 3, p. 031702, Jun. 2009. 
ANEXO A

Valores Críticos de $D\left(D_{\text {Critico }}\right)$ no Teste de Kolmogorov-Smirnov para uma amostra.

\begin{tabular}{|c|c|c|c|c|c|}
\hline \multirow{2}{*}{$\mathrm{N}$} & \multicolumn{5}{|c|}{$\begin{array}{c}\text { Nivel de Significância para } \\
D=\operatorname{máx}\left|F_{0}(X)-S_{N}(X)\right|\end{array}$} \\
\hline & 0,20 & 0,15 & 0,10 & 0,05 & 0,01 \\
\hline 1 & 0,900 & 0,925 & 0,950 & 0,975 & 0,995 \\
\hline 2 & 0,684 & 0,726 & 0,776 & 0,842 & 0,929 \\
\hline 3 & 0,565 & 0,597 & 0,642 & 0,708 & 0,828 \\
\hline 4 & 0,494 & 0,525 & 0,564 & 0,624 & 0,733 \\
\hline 5 & 0,446 & 0,474 & 0,510 & 0,565 & 0,669 \\
\hline 6 & 0,410 & 0,436 & 0,470 & 0,521 & 0,618 \\
\hline 7 & 0,381 & 0,405 & 0,438 & 0,486 & 0,577 \\
\hline 8 & 0,358 & 0,381 & 0,411 & 0,457 & 0,543 \\
\hline 9 & 0,339 & 0,360 & 0,388 & 0,432 & 0,514 \\
\hline 10 & 0,332 & 0,342 & 0,368 & 0,410 & 0,490 \\
\hline 11 & 0,307 & 0,326 & 0,352 & 0,391 & 0,468 \\
\hline 12 & 0,295 & 0,313 & 0,338 & 0,375 & 0,450 \\
\hline 13 & 0,284 & 0,302 & 0,325 & 0,361 & 0,433 \\
\hline 14 & 0,274 & 0,292 & 0,314 & 0,349 & 0,418 \\
\hline 15 & 0,266 & 0,283 & 0,304 & 0,338 & 0,404 \\
\hline 16 & 0,258 & 0,274 & 0,295 & 0,328 & 0,392 \\
\hline 17 & 0,250 & 0,266 & 0,286 & 0,318 & 0,381 \\
\hline 18 & 0,244 & 0,259 & 0,278 & 0,309 & 0,371 \\
\hline 19 & 0,237 & 0,252 & 0,272 & 0,301 & 0,363 \\
\hline 20 & 0,23 I & 0,246 & 0,264 & 0,294 & 0,356 \\
\hline 25 & 0,21 & 0,22 & 0,24 & 0,27 & 0,32 \\
\hline 30 & 0,19 & 0,20 & 0,22 & 0,24 & 0,29 \\
\hline 35 & 0,18 & 0,19 & 0,21 & 0,23 & 0,27 \\
\hline Mais de 35 & $\frac{1,07}{\sqrt{N}}$ & $\frac{1,14}{\sqrt{N}}$ & $\frac{1,22}{\sqrt{N}}$ & $\frac{1,36}{\sqrt{N}}$ & $\frac{0,63}{\sqrt{N}}$ \\
\hline
\end{tabular}

